Draft VERsion July 7, 2021

Preprint typeset using LATEX style emulateapj v. 8/13/10

\title{
ALMA MULTI-LINE OBSERVATIONS OF THE IR-BRIGHT MERGER VV 114
}

\author{
Toshiki Saito ${ }^{1,2}$, Daisuke Iono ${ }^{2,3}$, Min S. Yun ${ }^{4}$, Junko Ueda ${ }^{2}$, Kouichiro Nakanishi ${ }^{2,} 3,5$, Hajime Sugai ${ }^{6}$, Daniel \\ Espada $^{2,5}$, Masatoshi Imanishi ${ }^{2,3}, 7$, Kentaro Motohara ${ }^{8}$, Yosiaki Hagiwara ${ }^{2}$, Ken Tateuchi ${ }^{8}$, Minju Lee ${ }^{1,2}$ and \\ RYOHEI KAWABE ${ }^{1,2,3}$ \\ ${ }^{1}$ Department of Astronomy, Graduate school of Science, The University of Tokyo, 7-3-1 Hongo, Bunkyo-ku, Tokyo 133-0033, Japan \\ ${ }^{2}$ National Astronomical Observatory of Japan, 2-21-1 Osawa, Mitaka, Tokyo, 181-8588, Japan \\ ${ }^{3}$ The Graduate University for Advanced Studies (SOKENDAI), 2-21-1 Osawa, Mitaka, Tokyo 181-0015, Japan \\ ${ }^{4}$ Department of Astronomy, University of Massachusetts, Amherst, MA 01003, USA \\ ${ }^{5}$ Joint ALMA Observatory, Alonso de Córdova 3107, Vitacura, Casilla 19001, Santiago 19, Chile \\ ${ }^{6}$ Kavli Institute for the Physics and Mathematics of the Universe (WPI), The University of Tokyo, 5-1-5 Kashiwanoha, Kashiwa, Chiba \\ 277-8583, Japan \\ ${ }^{7}$ Subaru Telescope, 650 North A'ohoku Place, Hilo, HI 96720, USA and \\ ${ }^{8}$ Institute of Astronomy, The University of Tokyo, 2-21-1 Osawa, Mitaka, Tokyo 181-0015, Japan \\ Draft version July 7, 2021
}

\begin{abstract}
We present ALMA cycle 0 observations of the molecular gas and dust in the IR-bright mid-stage merger VV 114 obtained at $160-800 \mathrm{pc}$ resolution. The main aim of this study is to investigate the distribution and kinematics of the cold/warm gas and to quantify the spatial variation of the excitation conditions across the two merging disks. The data contain 10 molecular lines, including the first detection of extranuclear $\mathrm{CH}_{3} \mathrm{OH}$ emission in interacting galaxies, as well as continuum emission. We map the ${ }^{12} \mathrm{CO}(3-2) /{ }^{12} \mathrm{CO}(1-0)$ and the ${ }^{12} \mathrm{CO}(1-0) /{ }^{13} \mathrm{CO}(1-0)$ line ratio at $800 \mathrm{pc}$ resolution (in the units of $\mathrm{K} \mathrm{km} \mathrm{s}^{-1}$ ), and find that these ratios vary from $0.2-0.8$ and $5-50$, respectively. Conversely, the $200 \mathrm{pc}$ resolution $\mathrm{HCN}(4-3) / \mathrm{HCO}^{+}(4-3)$ line ratio shows low values $(<0.5)$ at a filament across the disks except for the unresolved eastern nucleus which is three times higher $(1.34$ $\pm 0.09)$. We conclude from our observations and a radiative transfer analysis that the molecular gas in the VV 114 system consists of five components with different physical and chemical conditions; i.e., 1) dust-enshrouded nuclear starbursts and/or AGN, 2) wide-spread star forming dense gas, 3) merger-induced shocked gas, 4) quiescent tenuous gas arms without star formation, 5) $\mathrm{H}_{2}$ gas mass of $(3.8 \pm 0.7) \times 10^{7} \mathrm{M}_{\odot}$ (assuming a conversion factor of $\left.\alpha_{\mathrm{CO}}=0.8 \mathrm{M}_{\odot}\left(\mathrm{K} \mathrm{km} \mathrm{s}^{-1} \mathrm{pc}^{2}\right)^{-1}\right)$ at the tip of the southern tidal arm, as a potential site of tidal dwarf galaxy formation.

Subject headings: galaxies: individual (VV 114, IC 1623, Arp 236) — galaxies: interactions — galaxies: starburst — galaxies: nuclei - ISM: molecules
\end{abstract}

\section{INTRODUCTION}

Galaxy interactions and mergers play important roles in triggering star formation and/or fueling the nuclear activity in the merging host galaxies (Hopkins et al. 2006). Recent high resolution simulations of major mergers show that large scale tidal forces as well as small scale turbulence and stellar feedback can significantly influence the distribution of gas, forming massive clumps of dense gas with $M_{\mathrm{H}_{2}}=10^{6}-10^{8} \mathrm{M}_{\odot}$ (e.g., Teyssier et al. 2010; Hopkins et al. 2013). These simulations also predict that the star formation not only increases as the galaxies first collide, but it also persists at a higher rate throughout the merger process, peaking at the final coalescence.

(Ultra-)Luminous Infrared Galaxies (U/LIRGs; Soifer et al. 1987) at low redshifts are almost exclusively strongly interacting and merging systems (Kartaltepe et al. 2010), often found at the mid to final stages of the merger. The elevated level of infrared luminosity originates from the reprocessed emission from the dust particles surrounding the starburst or the Active Galactic Nuclei (AGNs), both of which are likely triggered by the tidal interaction. The highest gas surface densities $\left(\Sigma_{\mathrm{H}_{2}}=5.4 \times 10^{4}-1.4 \times 10^{5} \mathrm{M}_{\odot} \mathrm{pc}^{-2}\right)$ and conse-

toshiki.saito@nao.ac.jp quently the highest star formation activities $\left(\Sigma_{\mathrm{SFR}}=\sim\right.$ $1000 \mathrm{M}_{\odot} \mathrm{yr}^{-1} \mathrm{kpc}^{-2}$ ) are usually found near the compact nuclear region (e.g., Arp220, NGC 6240; Downes \& Solomon 1998; Engel et al. 2010; Wilson et al. 2014). Dense molecular gas $\left(n \sim 10^{5}-10^{7} \mathrm{~cm}^{-3}\right)$ in U/LIRGs directly shows nuclear gas distribution and kinematics (e.g., Iono et al. 2004; Sakamoto et al. 2014). They are often surrounded by diffuse gas $\left(n \sim 10^{2}-10^{3} \mathrm{~cm}^{-3}\right)$ that may or may not be directly associated with star formation activities.

It has been demonstrated that the $\mathrm{HCN}(4-3)$ and $\mathrm{HCO}^{+}(4-3)$ emission lines, whose critical densities are $8.5 \times 10^{6}$ and $1.8 \times 10^{6} \mathrm{~cm}^{-3}$, respectively, can be used as tracers of the dense gas (e.g., Iono et al. 2013; GarciaBurillo et al. 2014; Imanishi \& Nakanishi 2014). On the other hand, $\mathrm{CO}(1-0)$ and ${ }^{13} \mathrm{CO}(1-0)$ line emission, whose critical densities are $4.1 \times 10^{2}$ and $1.5 \times 10^{3}$ $\mathrm{cm}^{-3}$, respectively, have been used extensively for tracing the global gas distribution and kinematics in merging U/LIRGs (e.g., Yun et al. 1994; Iono et al. 2004; Ueda et al. 2014). In addition, the ratio of these lines (e.g. ${ }^{12} \mathrm{CO} /{ }^{13} \mathrm{CO}$ and $\mathrm{HCN} / \mathrm{HCO}^{+}$) have been used to investigate the properties of the ISM (Casoli et al. 1992; Aalto et al. 1997) or to search for buried AGNs (e.g., Imanishi et al. 2007; Imanishi \& Nakanishi 2014). Limitations in 


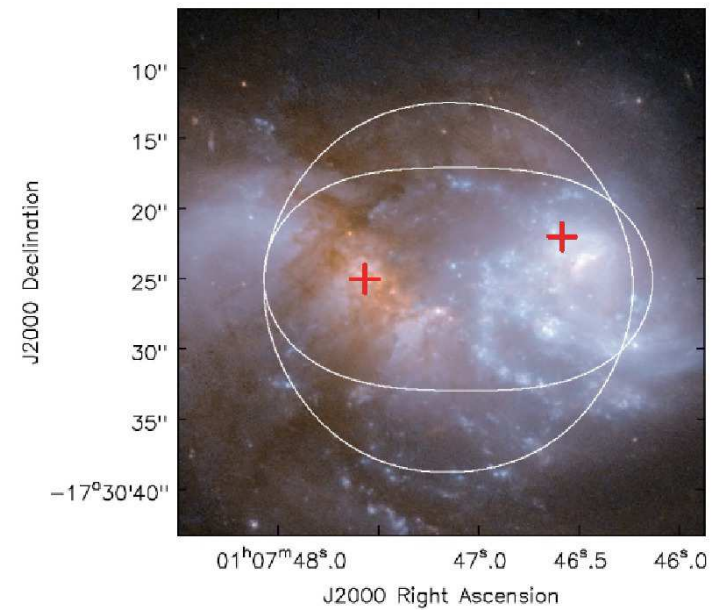

FIG. 1. - The HST/ACS image of VV 114 (Evans 2008). There is a dust lane from north to south in front of the eastern galaxy. The red crosses show the positions of the nuclei defined by the peak positions of the Ks-band observation (Tateuchi et al. 2012). The white ellipse shows a field of view of 3-point mosaic observation with band 7 , while the white circle shows a field of view of 7 -point mosaic observation with band 7 (see $\S 2$ ).

sensitivity and angular resolution have been the major obstacles in understanding the detailed distribution and kinematics of both dense and diffuse gas, and investigating the spatial variation of the line ratios and the physical condition of gas.

In this paper, we present Atacama Large Millimeter/submillimeter Array (ALMA) cycle 0 observations of the IR-bright merging galaxy VV 114. VV 114 is one of the best samples for studying the gas response during the critical stage when the two gas disks merge (Iono et al. 2005; Wilson et al. 2008). The target molecular lines include ${ }^{12} \mathrm{CO}(1-0),{ }^{13} \mathrm{CO}(1-0),{ }^{12} \mathrm{CO}(3-2)$, HCN $(4-$ $3)^{2}$ and $\mathrm{HCO}^{+}(4-3)$, and we also present the maps of $\mathrm{CH}_{3} \mathrm{OH}\left(2_{k}-1_{k}\right)$, CS $(2-1), \mathrm{CN}\left(1_{1 / 2}-0_{1 / 2}\right), \mathrm{CN}\left(1_{3 / 2}-\right.$ $0_{1 / 2}$ ), and CS (7-6) lines which were observed simultaneously within the same band. The main aim of this study is to investigate the distribution and kinematics of the diffuse and dense molecular gas and to quantify the spatial variation of the excitation conditions across the two merging disks.

VV 114 is a gas-rich $\left(M_{\mathrm{H}_{2}}=5.1 \times 10^{10} \mathrm{M}_{\odot}\right.$; Yun et al. 1994) nearby $\left(\mathrm{D}=82 \mathrm{Mpc} ; 1^{\prime \prime} 0=400 \mathrm{pc}\right)$ interacting system (Figure 1) with high-infrared luminosity $\left(L_{\mathrm{IR}}=4.7 \times 10^{11} L_{\odot} ;\right.$ Armus et al. 2009). The projected nuclear separation between the two optical galaxies (VV 114E and VV 114W) is about $6 \mathrm{kpc}$. Frayer et al. (1999) found a large amount of dust $\left(M_{\text {dust }}=1.2\right.$ $\left.\times 10^{8} \mathrm{M}_{\odot}\right)$ distributed across the system with a dust temperature of $20-25 \mathrm{~K}$. About half of the warmer dust traced in the mid-IR (MIR) is associated with the eastern galaxy, where both compact (nuclear region) and extended emission is found (Le Floc'h et al. 2002). Rich et al. (2011) found a bimodal distribution of velocity dispersions of several atomic forbidden lines and emission line ratios indicative of composite activity explained by a combination of wide-spread shocks and star formation. The wide-spread star formation is also revealed by $\mathrm{Pa} \alpha$ observation using ANIR camera mounted on miniTAO (Tateuchi et al. 2012, see also Appendix A.1). Iono et al. (2013) (hereafter paper I) identified a highly obscured AGN and compact starburst clumps using sub-arcsecond resolution ALMA cycle 0 observations of $\mathrm{HCN}(4-3)$ and $\mathrm{HCO}^{+}(4-3)$ emission.

This paper is organized as follows. We describe our observations and data reduction in $\S 2$, and results in $\S 3$. In $\S 4$ and $\S 5$, we provide molecular line ratios and physical parameters, such as the gas/dust mass, the gas temperature, and the gas density. In $\S 6$, we present the properties of "dense" gas ( $\S 6.1$ ), the comparison between molecular gas and star formation ( $\$ 6.2)$, the discussions of the $\mathrm{CO}$ isotope enhancement ( $(6.3)$, the gas-to-dust mass ratio (§6.4), the fractional abundances of $\mathrm{CS}, \mathrm{CH}_{3} \mathrm{OH}$, and $\mathrm{CN}$ relative to $\mathrm{H}_{2}(\S 6.5)$, and a potential tidal dwarf galaxy formations at the tip of the tidal arms of VV 114 ( $(6.6)$. We summarize and conclude this paper in $\S 7$. Throughout this paper, we adopt $\mathrm{H}_{0}=73 \mathrm{~km} \mathrm{~s}^{-1} \mathrm{Mpc}^{-1}, \Omega_{\mathrm{M}}=$ 0.27 , and $\Omega_{\Delta}=0.73$.

\section{OBSERVATIONS AND DATA REDUCTION}

Observations toward VV 114 were carried out as an ALMA cycle 0 program $(\mathrm{ID}=2011.0 .00467 . \mathrm{S} ; \mathrm{PI}=\mathrm{D}$. Iono) using fourteen - twenty $12 \mathrm{~m}$ antennas. The band 3 and band 7 receivers were tuned to the ${ }^{12} \mathrm{CO}(1-0)$, ${ }^{13} \mathrm{CO}(1-0),{ }^{12} \mathrm{CO}(3-2), \mathrm{HCN}(4-3)$, and $\mathrm{HCO}^{+}(4-$ 3) line emissions in the upper side band (see Table 1). The ${ }^{12} \mathrm{CO}(1-0)$ data were obtained on November 6, 2011 and May 4, 2012 in the compact and extended configurations, respectively. The ${ }^{13} \mathrm{CO}(1-0)$ data were obtained on May 27 and July 2, 2012 in the compact configuration. The ${ }^{12} \mathrm{CO}(3-2)$ emission was observed on November 5 , 2011 in the compact configuration (7-point mosaic). The $\mathrm{HCN}(4-3)$ and $\mathrm{HCO}^{+}(4-3)$ data were obtained on July 1, 2, and 3, 2012 in the extended configuration (3-point mosaic), simultaneously. Each spectral window had a bandwidth of $1.875 \mathrm{GHz}$ with 3840 channels, and two spectral windows were set to each sideband to achieve a total frequency coverage of $\sim 7.5 \mathrm{GHz}$ in these observations. The spectral resolution was $0.488 \mathrm{MHz}$ per channel. J1924-292, J0132-169, Uranus (Neptune for band 3 observations) were used for bandpass, phase, and flux calibrations. Detailed observational parameters are shown in Table 1.

We used the delivered calibrated data and mapping was accomplished using the clean task in CASA (McMullin et al. 2007). We made the data cubes with a velocity width of $5 \mathrm{~km} \mathrm{~s}^{-1}$ for the ${ }^{12} \mathrm{CO}$ line and $30 \mathrm{~km}$ $\mathrm{s}^{-1}$ for the other lines. All maps in this paper, except for ${ }^{12} \mathrm{CO}(3-2)$, are reconstructed with a Briggs weighting (robust $=0.5$; Briggs \& Cornwell 1992) and analyzed with MIRIAD and AIPS. The ${ }^{12} \mathrm{CO}(3-2)$ images are created with uniform weighting (see §3.2). The synthesized beam size of the ${ }^{12} \mathrm{CO}(1-0),{ }^{13} \mathrm{CO}(1-0),{ }^{12} \mathrm{CO}(3-2)$, and $\mathrm{HCN}(4-3)$ were 1 1'.97 $\times 1$ 1! 35 (P.A. $=82.3$ deg.), $1^{\prime \prime} .77 \times 1$ 1". 20 (P.A. $=85.8$ deg.), $1^{\prime \prime} 64 \times 1$ 1". 17 (P.A. $=$ 112.6 deg. $)$, and $0^{\prime \prime} 46 \times 0^{\prime \prime} .38$ (P.A. $=51.5$ deg. $)$, respectively. We also detected $\mathrm{CN}\left(1_{3 / 2}-0_{1 / 2}\right)$, CN $\left(1_{1 / 2}-0_{1 / 2}\right)$, $\mathrm{CS}(2-1), \mathrm{CH}_{3} \mathrm{OH}\left(2_{k}-1_{k}\right)$, and $\mathrm{CS}(7-6)$ line emission for the first time in VV 114. The properties of these molecular lines are summarized in Table 2. All images which we constructed are corrected for primary beam attenuation. The on-source times of band 3 and band 7 were about 40 minutes and 80 minutes, and the rms noise 

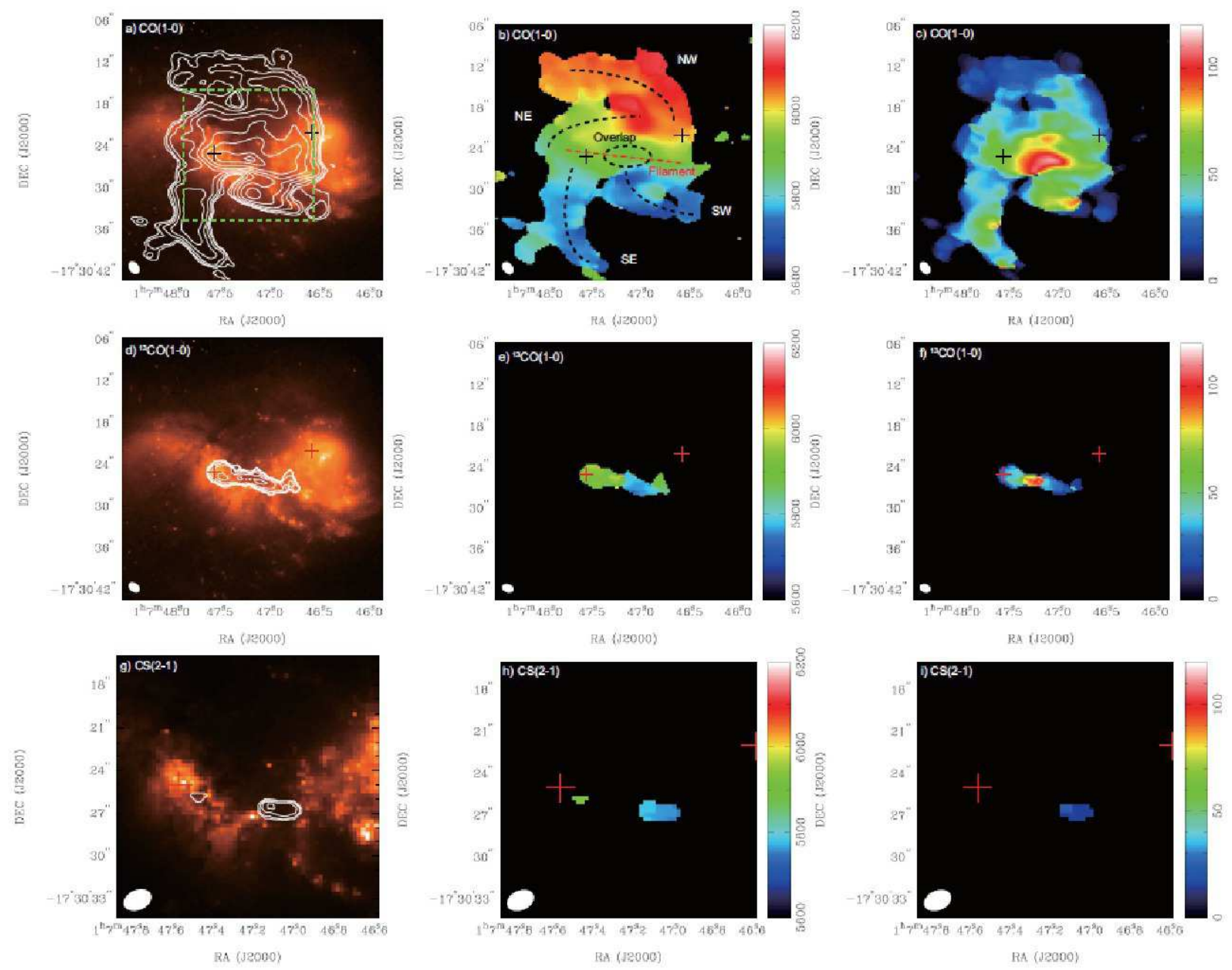

FIG. 2.- (a) ${ }^{12} \mathrm{CO}(1-0)$ integrated intensity image overlaid on the HST/ACS/F435W image of VV 114. The contours are 0.2, 0.4, 0.8, 1.6, 3.2, 6.4, 12.8, 25.6, and $33.0 \mathrm{Jy} \mathrm{km} \mathrm{s}{ }^{-1}$. The dashed green box shows an imaging field of other lines and continuum of this work except for the $\mathrm{CO}$ and ${ }^{13} \mathrm{CO}$ lines. (b) ${ }^{12} \mathrm{CO}(1-0)$ velocity field image. The velocity field image in color scale ranges from $5600 \mathrm{~km} \mathrm{~s} \mathrm{~s}^{-1}$ to $6200 \mathrm{~km} \mathrm{~s}^{-1}$. The dashed black lines represent tidal arms of VV 114. The dashed red line tracks the filamentary structure detected in images of other lines and dust continuum, and the dashed circle shows the overlap region. (c) ${ }^{12} \mathrm{CO}(1-0)$ velocity dispersion image. The velocity dispersion image in color scale ranges from $0 \mathrm{~km} \mathrm{~s}^{-1}$ to $120 \mathrm{~km} \mathrm{~s}^{-1}$. (d) The same as (a) but for ${ }^{13} \mathrm{CO}(1-0)$. The contours are $0.02,0.04,0.08,0.16,0.32$, and $0.64 \mathrm{Jy} \mathrm{km} \mathrm{s}^{-1}$. (e/f) The same as (b/c), respectively, but for ${ }^{13} \mathrm{CO}(1-0)$. (g) The same as (a) but for CS (2-1). The contours are 0.04, 0.08, 0.16, and $0.28 \mathrm{Jy} \mathrm{km} \mathrm{s}^{-1}$. (h/i) The same as (b/c), respectively, but for CS (2-1). The beam size of each line is shown in the bottom-left of the images (Table 2). The red crosses show the positions of the nuclei defined by the peak positions of the Ks-band observation (Tateuchi et al. 2012).

levels of the channel maps with $30 \mathrm{~km} \mathrm{~s}^{-1}$ resolution are $1.0 \mathrm{mJy}_{\text {beam }}^{-1}$ and $0.8 \mathrm{mJy}_{\text {beam }}^{-1}$, respectively. Furthermore, we made continuum maps at each observing frequency by adding the line-free channels. The rms level of the continuum images were $0.05 \mathrm{mJy}^{-1}$ beam $^{-1}$,

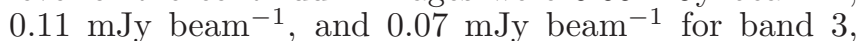
band 7 in the compact configuration, and band 7 in the extended configuration, respectively. The continuum emission was subtracted in the $u v$-plane before making the line images. Throughout this paper, the pixel scales of the band 3 and the band 7 images are set to $00^{\prime \prime} 3 /$ pixel and $0^{\prime \prime} 08 /$ pixel, respectively, and only the statistical error is considered unless mentioned otherwise. The systematic error on the absolute flux is estimated to be $\sim$ $5 \%$ and $\sim 10 \%$ for both sidebands in band 3 and band 7 , respectively.

In the following sections, we estimate the missing flux of each molecular line for which the single dish data are available in literature. Although the effect of missing flux becomes critical when we evaluate the global gas properties and the corresponding line ratios, the effect is negligible when we discuss structures that are smaller than the "maximum recoverable scale" (MRS) of each configuration of ALMA. This is estimated from the minimum baseline lengths of the assigned antenna configurations and the observed frequencies. The MRS of our observations are $\sim 8^{\prime \prime}$ and $\sim 7^{\prime \prime}$ in band 3 and band 7 , respectively (Table 1 ). Therefore the missing flux effect in this paper is negligible, since we derive physical parameters (e.g., molecular gas mass) only for structures smaller than $\sim 2^{\prime \prime}$.

\section{RESULTS}

Molecular line and continuum images are shown in Figures $2,3,4,5$, and 6 . The channel maps and the spectra of all line emissions are shown in Appendix A.2 and A.3. 

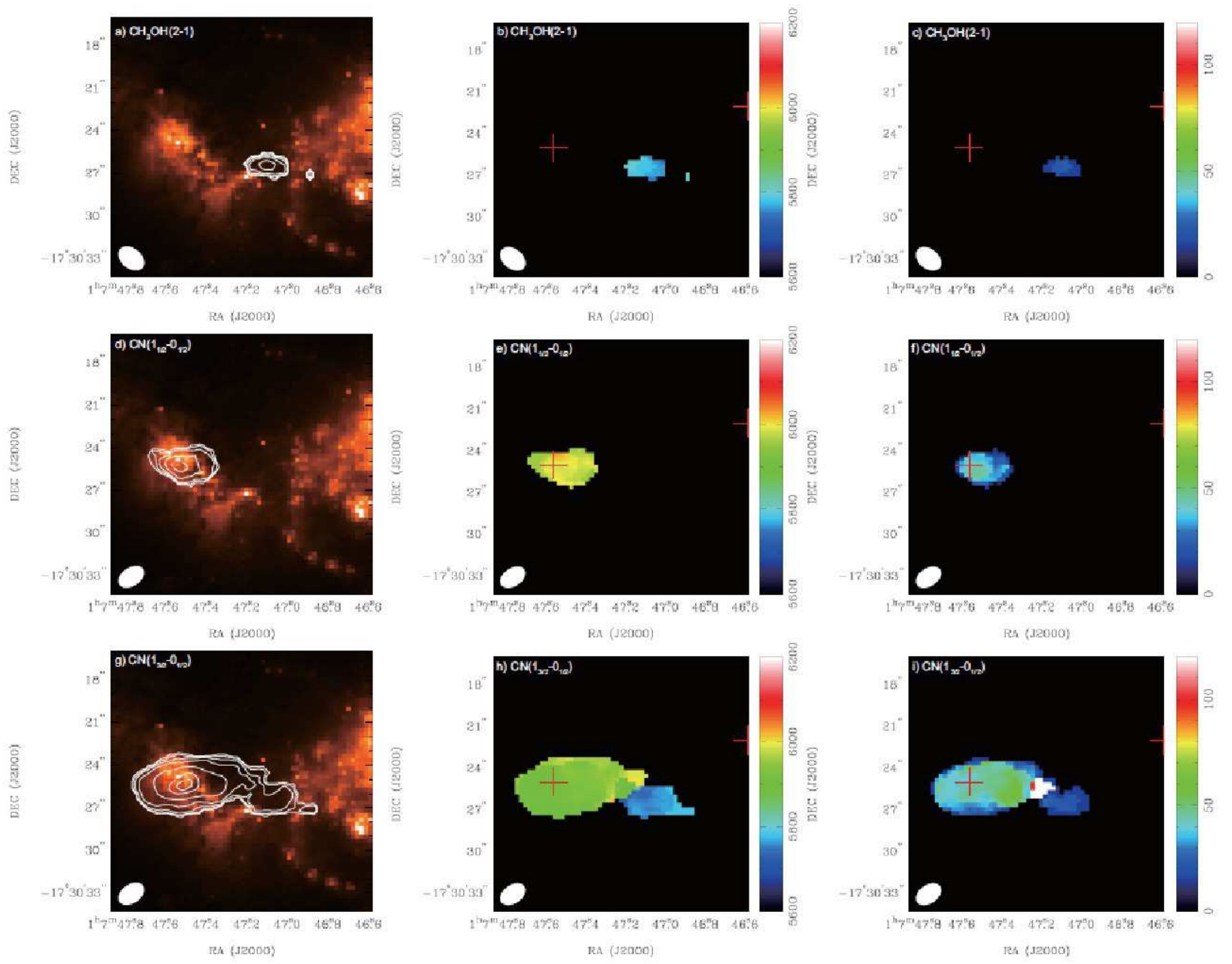

Fig. 3.- The same as Figure 2 but for (a, b, and c) $\mathrm{CH}_{3} \mathrm{OH}\left(2_{k}-1_{k}\right)$, (d, e, and f) $\mathrm{CN}\left(1_{1 / 2}-0_{1 / 2}\right)$, and (g, h, and i) CN $\left(1_{3 / 2}-0_{1 / 2}\right)$. (a) The contours are $0.02,0.04,0.08,0.16$, and $0.32 \mathrm{Jy} \mathrm{km} \mathrm{s}^{-1}$ (d) The contours are 0.04, 0.08, 0.16, 0.32, and $0.50 \mathrm{Jy} \mathrm{km} \mathrm{s}^{-1}$. (g) The contours are $0.04,0.08,0.16,0.32,0.64,1.00$, and $1.20 \mathrm{Jy} \mathrm{km} \mathrm{s}^{-1}$.

\subsection{Line Emissions in Band 3

$$
\text { 3.1.1. }{ }^{12} C O(1-0)
$$

The integrated intensity, velocity field, and velocity dispersion maps of VV 114 are shown in Figures 2a, $2 \mathrm{~b}$, and $2 \mathrm{c}$, respectively. The total ${ }^{12} \mathrm{CO}(1-0)$ integrated intensity of $\mathrm{VV} 114$ is $594.6 \pm 1.6 \mathrm{Jy} \mathrm{km} \mathrm{s}^{-1}$, which is 1.3 times larger than that detected using the NRAO $12 \mathrm{~m}$ telescope (461 Jy km s${ }^{-1}$; Sanders et al. 1991). This is because the pointing center for the NRAO $12 \mathrm{~m}$ observation was $25^{\prime \prime} 0$ southwest of the $\mathrm{CO}$ centroid identified from the ALMA map (NRAO $12 \mathrm{~m}$ : 01h07m45.7s, -17d30m36.5s; CO centroid: 01h07m47.2s, $-17 \mathrm{~d} 30 \mathrm{~m} 25.8 \mathrm{~s})$. At the adopted distance of VV 114 $(86 \mathrm{Mpc})$, the $1^{\prime \prime} .97 \times 1^{\prime \prime} .35$ beam of the ${ }^{12} \mathrm{CO}(1-0)$ observation gives us a resolution of $790 \mathrm{pc} \times 540 \mathrm{pc}$. The two crosses shown in all images represent the peaks obtained from the miniTAO/ANIR $K$ s-band observation, and we regard them as the progenitor's nuclei.

The integrated ${ }^{12} \mathrm{CO}$ (1-0) intensity map of VV 114 (Figure 2a) shows that the diffuse/cold gas forms two arm-like structures and a filamentary structure located at the center of the image. The global gas distribution is consistent with the previous ${ }^{12} \mathrm{CO}(1-0)$ observations
(Yun et al. 1994). The southeastern (SE) arm clearly follows the tidal arm seen in the HST/ACS image (Figure 1; Evans 2008), while the northwestern (NW) arm has no counterpart in any other wavelengths. The region from the center of VV 114 to the eastern nucleus shows a strong concentration of molecular gas $(\simeq 5$ ". 5 west of the eastern nucleus), and we refer to this region as the "overlap" region with a molecular "filament" (see Figure 2).

The ${ }^{12} \mathrm{CO}(1-0)$ velocity field map of VV 114 (Figure $2 \mathrm{~b}$ ) shows a significantly broad velocity range across the galaxy disks $\left(\simeq 600 \mathrm{~km} \mathrm{~s}^{-1}\right)$. The SE arm has a blueshifted velocity from $5650 \mathrm{~km} \mathrm{~s}^{-1}$ to $5920 \mathrm{~km} \mathrm{~s}^{-1}$, while the NW arm has a red-shifted velocity from $5950 \mathrm{~km} \mathrm{~s}^{-1}$ to $6160 \mathrm{~km} \mathrm{~s}^{-1}$. One possibility for the larger velocity width in the SE arm may be a highly inclined tidal arm. Two other arm-like features are also detected in the ${ }^{12} \mathrm{CO}(1-0)$ observations. One arm is located $\simeq 4$.' 0 northeast of the eastern nucleus and shows an arc around the eastern nucleus in the velocity range of $5810 \mathrm{~km} \mathrm{~s}^{-1}$ to $6180 \mathrm{~km} \mathrm{~s}^{-1}$. The other arm is located $\simeq 10^{\prime \prime} .0$ west of the SE arm and has a strong peak $(\simeq 262.5 \pm$ $1.0 \mathrm{Jy} \mathrm{km} \mathrm{s}^{-1}$ ) in the velocity range of $5610 \mathrm{~km} \mathrm{~s}^{-1}$ to 

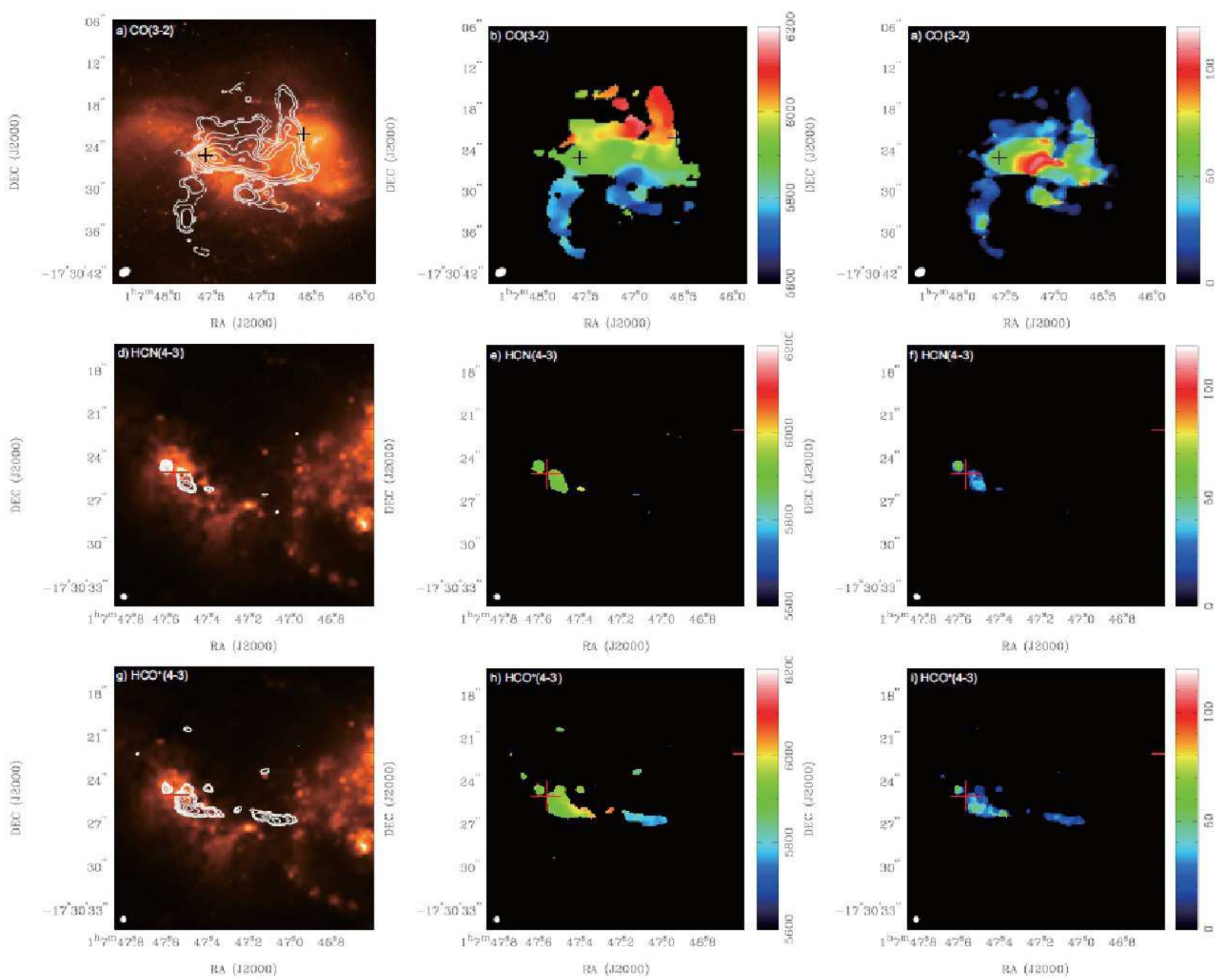

Fig. 4.- The same as Figure 2 but for (a, b, and c) ${ }^{12} \mathrm{CO}(3-2)$, (d, e, and f) HCN (4-3), and (g, h, and i) $\mathrm{HCO}^{+}(4-3)$. (a) The contours are $2,4,8,16,32,64,128$, and $170 \mathrm{Jy} \mathrm{km} \mathrm{s}^{-1}$ (d) The contours are 0.04, 0.08, 0.16, 0.32, 0.64, 1.28, and 1.80 Jy km s $\mathrm{s}^{-1}$. (g) The contours are $0.04,0.08,0.16,0.32,0.64,1.28$, and $2.40 \mathrm{Jy} \mathrm{km} \mathrm{s}{ }^{-1}$.

\section{$5900 \mathrm{~km} \mathrm{~s}^{-1}$.}

The overlap region has the highest velocity dispersion $\left(\simeq 110 \mathrm{~km} \mathrm{~s}^{-1}\right)$ (Figure $2 \mathrm{c}$ ). The NW arm has an average velocity dispersion of $\simeq 30 \mathrm{~km} \mathrm{~s}^{-1}$, while the $\mathrm{SE}$ arm has $\simeq 40 \mathrm{~km} \mathrm{~s}^{-1}$. These values are significantly higher than the dispersions seen in Giant Molecular Clouds (GMCs) in the LMC (2-14 $\mathrm{km} \mathrm{s}^{-1}$; Minamidani et al. 2008; Fujii et al. 2014) and slightly higher than that in Giant Molecular Associations (GMAs) in the Antennae galaxy (6$36 \mathrm{~km} \mathrm{~s}^{-1}$; Ueda et al. 2012). We suggest that the main contribution to the ${ }^{12} \mathrm{CO}(1-0)$ velocity dispersion is inter cloud turbulent medium along the tidal arm, and/or shocked region induced by the tidal interaction, rather than the velocity dispersion of the GMCs/GMAs.

\subsection{2. ${ }^{13} \mathrm{CO}(1-0)$}

The integrated intensity, velocity field, and velocity dispersion maps of ${ }^{13} \mathrm{CO}(1-0)$ are shown in Figures $2 \mathrm{~d}$, $2 \mathrm{e}$, and $2 \mathrm{f}$, respectively. The integrated ${ }^{13} \mathrm{CO}(1-0)$ intensity map of VV 114 (Figure 2d) shows a filamentary structure across the galaxy disks, which is consistent with the region where the ${ }^{12} \mathrm{CO}(1-0)$ filament is detected. The total ${ }^{13} \mathrm{CO}(1-0)$ integrated intensity is 5.9 $\pm 0.4 \mathrm{Jy} \mathrm{km} \mathrm{s}{ }^{-1}$. The strongest peak is located $\simeq 4$ ". 2 southwest of the eastern nucleus. The ${ }^{13} \mathrm{CO}(1-0)$ velocity field map of VV 114 (Figure 2e) shows a narrower velocity range $\left(5670-6000 \mathrm{~km} \mathrm{~s}^{-1}\right)$ than that of the ${ }^{12} \mathrm{CO}(1-0)$ emission $\left(5600-6200 \mathrm{~km} \mathrm{~s}^{-1}\right)$. This suggests that the ${ }^{13} \mathrm{CO}(1-0)$ emission mainly comes from two components, the eastern galaxy and the blue-shifted component of the overlap region. The ${ }^{13} \mathrm{CO}(1-0)$ velocity dispersion map of VV 114 (Figure 2f) shows a high velocity dispersion component $\left(\sim 100 \mathrm{~km} \mathrm{~s}^{-1}\right)$ between the eastern nucleus and the overlap region. This significant velocity dispersion may be caused by a superposition of clouds (see the double-peak spectrum at R39 shown in Appendix A.3).

\subsection{3. $\mathrm{CS}(2-1)$ and $\mathrm{CH}_{3} \mathrm{OH}\left(2_{k}-1_{k}\right)$}

The CS $(2-1)$ and $\mathrm{CH}_{3} \mathrm{OH}\left(2_{k}-1_{k}\right)$ lines are only detected at the overlap region (Figures $2 \mathrm{~g}, 2 \mathrm{~h}, 2 \mathrm{i}, 3 \mathrm{a}, 3 \mathrm{~b}$, and 3c). This is the first detection of the $\mathrm{CH}_{3} \mathrm{OH}\left(2_{k}-\right.$ $1_{k}$ ) emission in a merger-induced overlap region. We observed the blended set of $2_{1}-1_{1}\left(\nu_{\text {rest }}=96.756 \mathrm{GHz}\right.$, $\left.E_{\text {up }} / k=28.0 \mathrm{~K}\right), 2_{0}-1_{0} E\left(\nu_{\text {rest }}=96.745 \mathrm{GHz}, E_{\text {up }} / k\right.$ $=20.1 \mathrm{~K}), 2_{0}-1_{0} A^{+}\left(\nu_{\text {rest }}=96.741 \mathrm{GHz}, E_{\text {up }} / k=\right.$ $7.0 \mathrm{~K})$, and $2_{-1}-1_{-1} E\left(\nu_{\text {rest }}=96.739 \mathrm{GHz}, E_{\text {up }} / k=\right.$ 


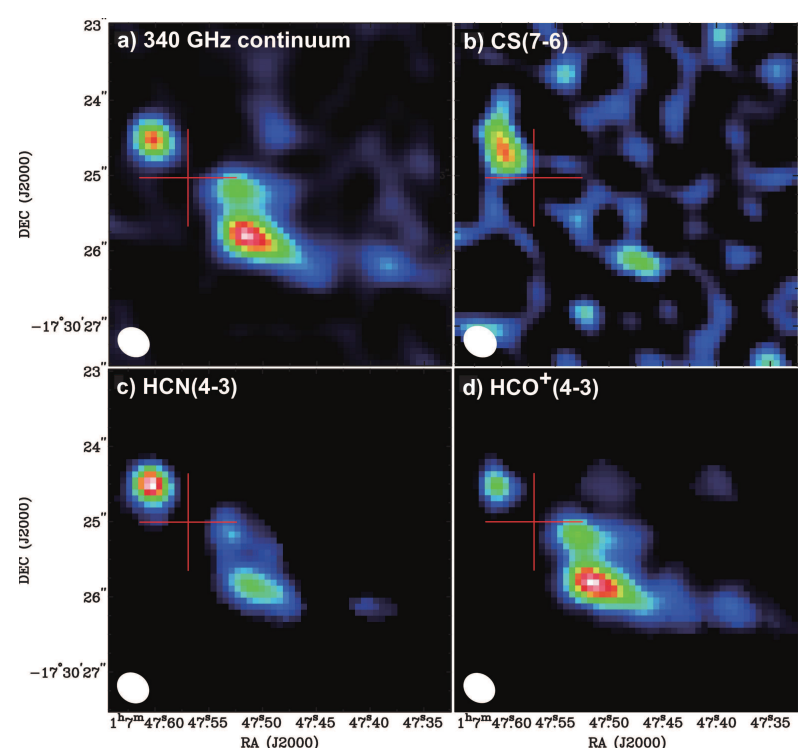

FIG. 5.- (a) $340 \mathrm{GHz}$ continuum flux image of VV 114E. The flux in color scale ranges from $0 \mathrm{mJy}^{\text {beam }}{ }^{-1}$ to $3.4 \mathrm{mJy}^{\mathrm{beam}}{ }^{-1}$. (b) CS (7-6) integrated intensity image of VV $114 \mathrm{E}$. The flux in color scale ranges from $0 \mathrm{Jy} \mathrm{km} \mathrm{s}^{-1}$ to $0.45 \mathrm{Jy} \mathrm{km} \mathrm{s}^{-1}$. (c) $\mathrm{HCN}$ (43 ) integrated intensity image of VV 114E. The flux in color scale ranges from $0 \mathrm{Jy} \mathrm{km} \mathrm{s}^{-1}$ to $2.0 \mathrm{Jy} \mathrm{km} \mathrm{s}^{-1}$. (d) $\mathrm{HCO}^{+}(4-3)$ integrated intensity image of $\mathrm{VV} 114 \mathrm{E}$. The flux in color scale ranges from $0 \mathrm{Jy} \mathrm{km} \mathrm{s}{ }^{-1}$ to $2.8 \mathrm{Jy} \mathrm{km} \mathrm{s}{ }^{-1}$. The beam size of each line and continuum is shown in the bottom-left of the images (Table 2). The red cross shows the position of the eastern nucleus defined by the peak position of the Ks-band observation (Tateuchi et al. 2012).

12.5 K), thermal transitions of $\mathrm{CH}_{3} \mathrm{OH}$ (hereafter designated the $2_{k}-1_{k}$ transition). The distribution of these molecular lines is clearly different from the other dense gas tracers detected in the current program. The peaks of $\mathrm{CS}(2-1)$ and $\mathrm{CH}_{3} \mathrm{OH}\left(2_{k}-1_{k}\right)$ are coincident with one of the peaks of ${ }^{13} \mathrm{CO}(1-0)$ to within $0^{\prime \prime} 5$. The total CS $(2$ 1) and $\mathrm{CH}_{3} \mathrm{OH}\left(2_{k}-1_{k}\right)$ integrated intensities are $0.4 \pm$ $0.1 \mathrm{Jy} \mathrm{km} \mathrm{s}^{-1}$ and $0.5 \pm 0.1 \mathrm{Jy} \mathrm{km} \mathrm{s}^{-1}$, respectively. The signal to noise is too low to resolve the velocity structure.

\subsection{4. $C N\left(1_{3 / 2}-0_{1 / 2}\right)$ and $C N\left(1_{1 / 2}-0_{1 / 2}\right)$}

Two radical CN rotational transitions $N=1-0(J=$ $3 / 2-1 / 2$ and $1 / 2-1 / 2)$ are detected at the eastern nucleus. The $J=3 / 2-1 / 2$ transition is extended toward the overlap region (Figures 3d, 3e, 3f, 3g, 3h, and 3i). We can not resolve their multiplet because of the coarse frequency resolution $\left(11.5 \mathrm{MHz} \simeq 30 \mathrm{~km} \mathrm{~s}^{-1}\right)$. Because the critical density of $\mathrm{CN}$ is high $\left(\sim 10^{6} \mathrm{~cm}^{-3}\right)$, the CN emission mainly comes from denser gas regions than regions traced by ${ }^{12} \mathrm{CO}(1-0)$. The $J=3 / 2-1 / 2$ transition shows a similar distribution to the ${ }^{13} \mathrm{CO}(1-0)$ emission, but it is less extended over the overlap region. The total CN $\left(1_{1 / 2}-0_{1 / 2}\right)$ and $\mathrm{CN}\left(1_{3 / 2}-0_{1 / 2}\right)$ integrated intensities are $2.0 \pm 0.1 \mathrm{Jy} \mathrm{km} \mathrm{s}^{-1}$ and $5.4 \pm 0.3 \mathrm{Jy} \mathrm{km} \mathrm{s}^{-1}$, respectively. The highest velocity dispersion in the $\mathrm{CN}\left(1_{3 / 2}{ }^{-}\right.$ $0_{1 / 2}$ ) image is also detected between the eastern nucleus and the overlap region, and this is likely caused by a superposition of clouds similar to the case of the ${ }^{13} \mathrm{CO}(1-0)$ image (see Appendix A.3).

\subsection{Line Emission in Band 7}

\subsection{1. ${ }^{12} \mathrm{CO}(3-2)$}

The ${ }^{12} \mathrm{CO}(3-2)$ emission maps are presented in Figure 4. The estimated missing flux in our ALMA observation is $21 \pm 1 \%$ (James Clerk Maxwell Telescope (JCMT): $2956 \pm 133 \mathrm{Jy} \mathrm{km} \mathrm{s}^{-1}$ and ALMA: $2343.7 \pm$ $4.7 \mathrm{Jy} \mathrm{km} \mathrm{s}^{-1}$; Wilson et al. 2008; Saito et al. 2013). Although our ${ }^{12} \mathrm{CO}(3-2)$ observation recovers more flux than the Submillimeter Array (SMA) observation (1530 $\pm 16 \mathrm{Jy} \mathrm{km} \mathrm{s}^{-1}$; the missing flux $=48 \pm 15 \%$; Wilson et al. 2008), there are significant negative sidelobes at the north and south of the image which is likely the cause of missing flux. We made the CLEANed image with a uniform $u v$ weighting to minimized the sidelobe level (Thompson et al. 2001).

The ${ }^{12} \mathrm{CO}(3-2)$ integrated intensity map of VV 114 (Figure 4a) shows two arm-like structures and a filamentary structure similar to the ${ }^{12} \mathrm{CO}(1-0)$ image, and the strongest peak is at $\simeq 5$ ". 5 west of the eastern nucleus. The global gas distribution is consistent with the previous ${ }^{12} \mathrm{CO}(3-2)$ observations (Iono et al. 2004; Wilson et al. 2008). The ${ }^{12} \mathrm{CO}(3-2)$ velocity field map of VV 114 (Figure 4b) also shows significant broad velocity range across the galaxy disks $\left(\simeq 600 \mathrm{~km} \mathrm{~s}^{-1}\right)$, similar to the ${ }^{12} \mathrm{CO}(1-0)$ velocity field map. The $\mathrm{SE}$ arm has a blueshifted velocity from $5650 \mathrm{~km} \mathrm{~s}^{-1}$ to $5920 \mathrm{~km} \mathrm{~s}^{-1}$, while the NW arm has a red-shifted velocity from $5950 \mathrm{~km} \mathrm{~s}^{-1}$ to $6160 \mathrm{~km} \mathrm{~s}^{-1}$. Other two arm-like features are also detected. One located $\simeq 4^{\prime \prime} 0$ northeast of the eastern nucleus shows an arc around the eastern nucleus and has red-shifted velocities from $5810 \mathrm{~km} \mathrm{~s}^{-1}$ to $6180 \mathrm{~km} \mathrm{~s}^{-1}$. This arm coincides with the NE arm detected in the ${ }^{12} \mathrm{CO}(1-0)$. The other one located at $\simeq 100^{\prime \prime} 0$ west of the $\mathrm{SE}$ arm has a strong peak $\left(\simeq 262.5 \pm 0.9 \mathrm{Jy} \mathrm{km} \mathrm{s}{ }^{-1}\right)$ and blue-shifted velocities from $5610 \mathrm{~km} \mathrm{~s}^{-1}$ to $5900 \mathrm{~km} \mathrm{~s}^{-1}$. This arm also coincide with the SW arm detected in the ${ }^{12} \mathrm{CO}(1-0)$. From the ${ }^{12} \mathrm{CO}(3-2)$ velocity dispersion map of VV 114 (Figure 4c), we find that the overlap region has the highest velocity dispersion $\left(\simeq 110 \mathrm{~km} \mathrm{~s}^{-1}\right)$. The velocity dispersion of the NW arm is $\simeq 30 \mathrm{~km} \mathrm{~s}^{-1}$, while the $\mathrm{SE}$ arm is $\simeq 60 \mathrm{~km} \mathrm{~s}^{-1}$.

\subsection{2. $\mathrm{HCN}(4-3)$ and $\mathrm{HCO}^{+}(4-3)$}

The $\mathrm{HCN}(4-3)$ and $\mathrm{HCO}^{+}(4-3)$ images are shown in Figures 4d, 4e, 4f, 4g, 4h, and 4i. While the HCN (4-3) emission is only seen near the eastern nucleus of VV 114 and is resolved into four peaks, the $\mathrm{HCO}^{+}(4-3)$ emission is more extended and has at least 10 peaks in the integrated intensity map. The total integrated intensities of $\mathrm{HCO}^{+}(4-3)$ and $\mathrm{HCN}(4-3)$ are $15.3 \pm 0.4 \mathrm{Jy} \mathrm{km}$ $\mathrm{s}^{-1}$ and $4.4 \pm 0.2 \mathrm{Jy} \mathrm{km} \mathrm{s}^{-1}$, respectively. The higher $\mathrm{HCO}^{+}(4-3)$ flux observed with the SMA $(17 \pm 2 \mathrm{mJy}$, Wilson et al. 2008) using a $2^{\prime \prime} .8 \times 22^{\prime \prime} 0$ beam is likely attributed to missing flux by the ALMA observation. A compact component in the eastern nucleus is unresolved with the current resolution, and the upper limit to the size is 200 pc. The HCN (4-3) emission is not detected in the overlap region, where both the high ${ }^{12} \mathrm{CO}(1-0)$ velocity dispersion and the significant $\mathrm{CH}_{3} \mathrm{OH}\left(2_{k}-1_{k}\right)$ and $\mathrm{HCO}^{+}(4-3)$ detection suggest the presence of shocked gas (Krips et al. 2008). We concluded in paper I from their source size, line widths, and the relative strengths of $\mathrm{HCN}(4-3)$ and $\mathrm{HCO}^{+}(4-3)$ that the unresolved eastern 

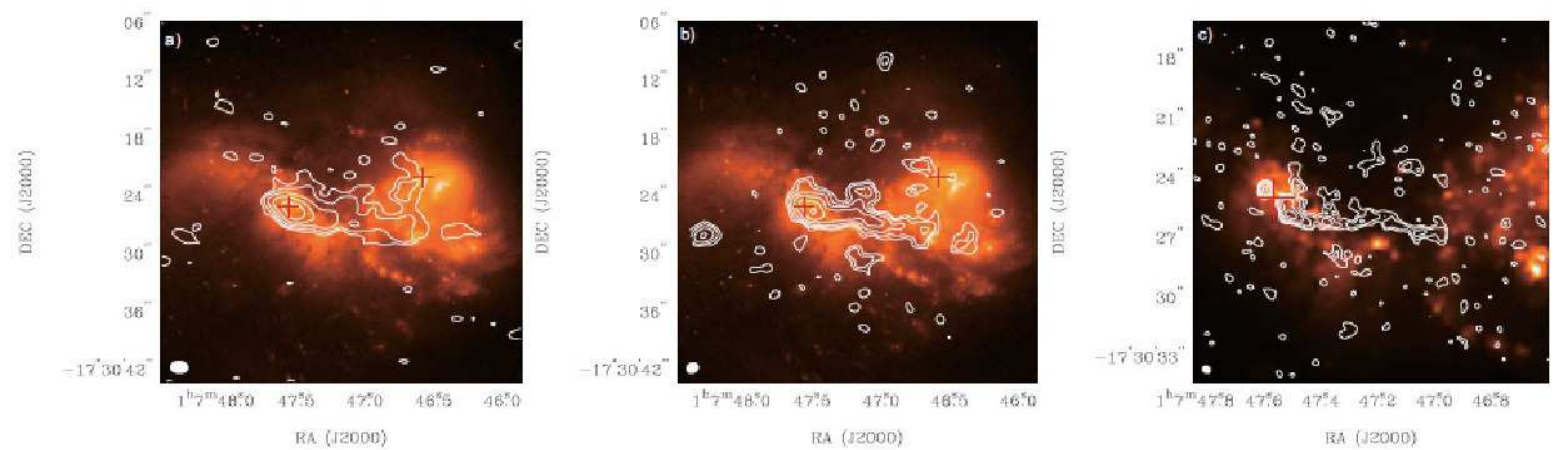

FiG. 6.- (a) The $110 \mathrm{GHz}$ continuum flux image overlaid on the HST/ACS/F435W image of VV 114. The contours are 0.10, 0.20, 0.40, 0.80 , and $1.60 \mathrm{mJy}$ beam $^{-1}$. The red crosses show the positions of the nuclei defined by the peak positions of the Ks-band observation (Tateuchi et al. 2012). (b) The low resolution $340 \mathrm{GHz}$ continuum flux image overlaid on the HST/ACS/F435W image of VV 114. The contours are $0.22,0.44,0.88,1.76,3.52$, and $7.04 \mathrm{mJy}_{\text {beam }}^{-1}$. A strong point source at the eastern edge of the image is a distant star-forming galaxy, ALMA J010748.3 - 173028 (see Tamura et al. 2014). (c) The high resolution 340 GHz continuum flux image overlaid on the HST/ACS/F435W image of VV 114. The contours are $0.14,0.28,0.56,1.12$, and $2.24 \mathrm{mJy}^{\text {beam }}{ }^{-1}$. The red cross shows the position of the eastern nucleus defined by the peak position of the Ks-band observation (Tateuchi et al. 2012). The beam size of each continuum is shown in the bottom-left of the images (Table 2).

nucleus harbors an obscured AGN, and the dense clumps in the western galaxy are related to extended starbursts.

\subsection{3. $C S(7-6)$}

The CS (7-6) emission has the highest critical density $\left(n_{\mathrm{cr}} \simeq 10^{7} \mathrm{~cm}^{-3}\right)$ of all of the lines detected in our observations. The $\mathrm{CS}(7-6)$ emission is marginally $(\mathrm{S} / \mathrm{N} \sim 4)$ detected at the eastern nucleus (Figure 5), and the total flux is $0.5 \pm 0.1 \mathrm{Jy} \mathrm{km} \mathrm{s}^{-1}$.

\subsection{Continuum Emission}

The continuum image at $110 \mathrm{GHz}$ shows a filamentary structure similar to the molecular line image (Figure 6a). We construct low resolution $\left(1^{\prime \prime} .33 \times 1\right.$ 1.'12) and high resolution $(0$.' $45 \times 0$ ". 38$)$ images of the $340 \mathrm{GHz}$ continuum (Figures 6b, and 6c) using the combined data (compact + extended) and the extended configuration data, respectively. We find that the filamentary structure and the unresolved eastern nucleus are both present in dust continuum. The total flux of the $110 \mathrm{GHz}$ and the low resolution $340 \mathrm{GHz}$ continuum emission are $10.3 \pm 0.2 \mathrm{mJy}$ and $38.6 \pm 0.3 \mathrm{mJy}$, respectively. The estimated missing flux relative to the JCMT $340 \mathrm{GHz}$ observation (Wilson et al. 2008) is $75 \pm 4 \%$ (SMA: $79 \pm 7 \%$ ). The difference in the recovered flux between ${ }^{12} \mathrm{CO}(3-2)$ and $340 \mathrm{GHz}$ continuum emission may be caused by the difference in the distribution. The $110 \mathrm{GHz}$ and $340 \mathrm{GHz}$ continuum emission is detected at the eastern nucleus $(\mathrm{S} / \mathrm{N} \sim 50$ and 70) and the filamentary structure $(\mathrm{S} / \mathrm{N} \sim 8$ and 24$)$ identified in the ${ }^{13} \mathrm{CO}(1-0)$ image, both with high significance.

\section{SPATIALLY RESOLVED LINE RATIOS}

We assign 39 "R" boxes $(2$ ". $0 \times 2$ ". 0 ; R1 - R39; see Figure 7 ) for the band 3 and ${ }^{12} \mathrm{CO}(3-2)$ data and 15 smaller "S" boxes $(1$ ". $2 \times 1$ ". 2 ; S0 - S14; see Figure 8$)$ for the rest of the data to estimate the physical parameters, such as the molecular gas mass $\left(M_{\mathrm{H}_{2}}\right)$, dense gas mass ( $\left.M_{\text {dense }}\right)$, dust mass $\left(M_{\text {dust }}\right)$, star formation rate $(\mathrm{SFR})$, kinetic temperature $\left(T_{\text {kin }}\right)$, gas density $\left(n_{\mathrm{H}_{2}}\right)$, gas column density $\left(N\left(\mathrm{H}_{2}\right)\right)$, and molecular abundance relative to $\mathrm{H}_{2}\left([\mathrm{X}] /\left[\mathrm{H}_{2}\right]\right)$. The positions of the boxes are chosen to cover the CO (3-2) emission (R1 - R39) and the $\mathrm{HCO}^{+}(4-3)$ emission ( $\left.\mathrm{S} 0-\mathrm{S} 14\right)$. The sizes of the boxes are chosen such that they are comparable to the beam size. Before deriving the parameters and line ratios at each box, we first matched the $u v$ range between our data set and reconstructed the integrated intensity image of each line. The shortest baseline lengths are set to $13.5 \mathrm{k} \lambda$ and $40.0 \mathrm{k} \lambda$ for the molecular lines in the band 3 and the band 7, respectively, and the images are convolved into the same resolution $\left(2\right.$.' $0 \times 11^{\prime \prime} 5$ with a P.A. of $83 \mathrm{deg}, 1^{\prime \prime} .2 \times 1$.! 0 with a P.A. of $\left.119 \mathrm{deg}\right)$. For each ratio, the two integrated intensity images were expressed in the units of $\mathrm{K} \mathrm{km} \mathrm{s}^{-1}$ before calculating the ratio at locations where both lines are detected above $3 \sigma$. The derived box-summed spectra are listed in Appendix A.3. We carried out a multi Gaussian fit (one - three components) to reproduce the box-summed spectra, and labeled the components as "a", "b", and "c" from the bluest peak (e.g., the bluest peak at R21 is labeled as R21a).

$$
\text { 4.1. }{ }^{12} C O \text { (3-2) } /{ }^{12} C O \text { (1-0), } R_{3-2 / 1-0}
$$

The ${ }^{12} \mathrm{CO}(3-2) /{ }^{12} \mathrm{CO}(1-0)$ ratio, $R_{3-2 / 1-0}$, can be used as an indicator of the dense/warm gas content relative to the total molecular gas. The $R_{3-2 / 1-0}$ of VV 114 varies from 0.2 to 0.8 , as shown in Figure 7 (left) and Table 6 . This range is larger than the same ratios derived for normal spirals, which is typically $0.15-0.5$ when observed with a similar linear resolution (Warren et al. 2010). At the edge and the center of the filament, $R_{3-2 / 1-0}$ is higher $(0.53-0.69)$ than the highest peaks of each arm $(\sim 0.4)$. This suggests that the CO emitting gas at the filament have higher excitation conditions than normal spirals, while the conditions of each arm of VV 114 are consistent with arms and nuclei of normal spirals. The $R_{3-2 / 1-0}$ at the eastern nucleus is $0.76 \pm$ 0.01 . It is suggested that the $R_{3-2 / 1-0}$ is much higher $(3.12 \pm 0.03$ in NGC 1068; Tsai et al. 2012) for gas surrounding an $\mathrm{AGN}$, and the low $R_{3-2 / 1-0}$ in VV 114 may be due to the difference in filling factor $(160 \times 140 \mathrm{pc}$ beam averaging for NGC 1068, while 800 pc box averaging for $\mathrm{VV}$ 114). It is possible, however, that the nuclear 

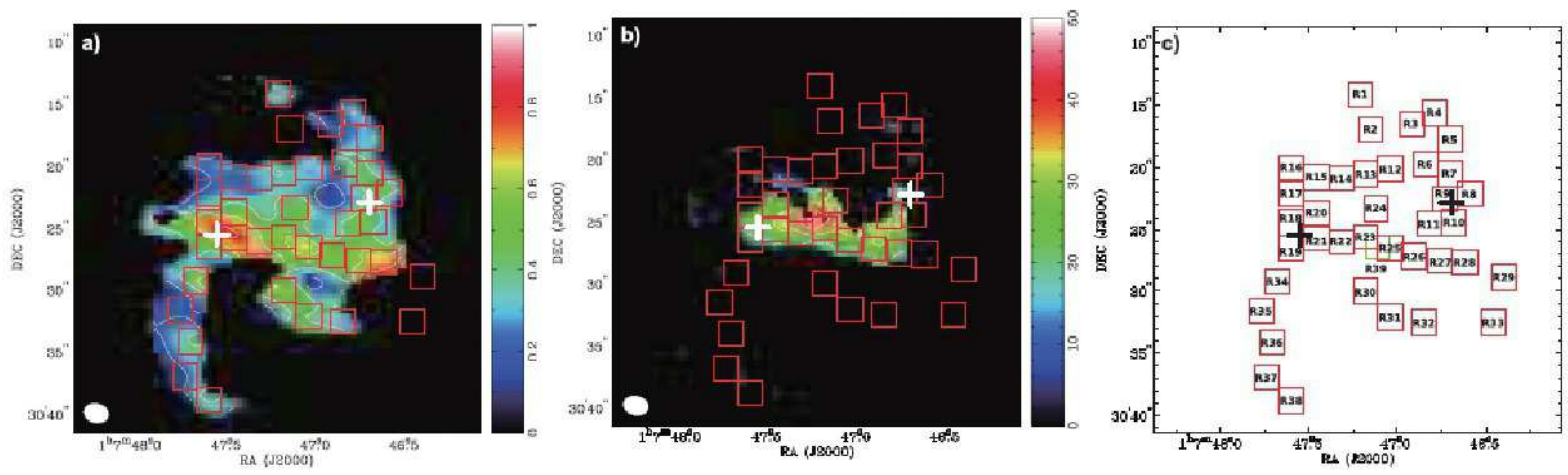

FIG. 7.- (a) The $R_{3-2 / 1-0}$ image. The ratio in color scale ranges from 0 to 1 . The white crosses show the positions of the nuclei defined by the peak positions of the Ks-band observation (Tateuchi et al. 2012). (b) The $R_{12 / 13}$ image. The ratio in color scale ranges from 0 to 40. (c) Locations of 39 boxes (R1 - R39) that are used to calculate the line ratios and physical parameters. For each ratio, the two integrated intensity images were convolved to the same resolution and expressed in units of $\mathrm{K} \mathrm{km} \mathrm{s}^{-1}$ before calculating the ratio at locations where both lines are detected above $3 \sigma$. The black crosses show the positions of the nuclei defined by the peak positions of the Ks-band observation (Tateuchi et al. 2012). The beam size of each line ratio is shown in the bottom-left of the images.

excitation conditions are different from source to source.

$$
\text { 4.2. }{ }^{12} C O(1-0) /{ }^{13} C O(1-0), R_{12 / 13}
$$

In general, the ${ }^{12} \mathrm{CO}$ lines has higher optical depths than the ${ }^{13} \mathrm{CO}(1-0)$ line. Therefore, the measured ${ }^{12} \mathrm{CO}(1-0) /{ }^{13} \mathrm{CO}(1-0)$ line intensity ratio, $R_{12 / 13}$, gives a lower limit to the $\mathrm{CO} /{ }^{13} \mathrm{CO}$ abundance ratio (hereafter $\left.[\mathrm{CO}] /\left[{ }^{13} \mathrm{CO}\right]\right)$. We present the $R_{12 / 13}$ image of $\mathrm{VV} 114$ in Figure 7 (center). The $R_{12 / 13}$ increases from the arms $(<17)$ to the filament $(15-32)$. Observationally, $R_{12 / 13}$ increases towards the central region of galaxies (Aalto et al. 1995), where the gas is generally warmer and denser. Aalto et al. (1995) suggest that the moderate optical depth of ${ }^{12} \mathrm{CO}(1-0)$ emission and/or the high $[\mathrm{CO}] /\left[{ }^{13} \mathrm{CO}\right]$ environment can increase the $R_{12 / 13}$ in nuclei of U/LIRGs. In order to understand which of the two (optical depths or abundances) is dominant, we calculated and mapped the optical depth of the ${ }^{12} \mathrm{CO}(1-0)$ and the ${ }^{13} \mathrm{CO}(1-0)$ as shown in Table 14 and Figure 10. We provide an interpretation of these results in $§ 5.2$.

\section{3. $\mathrm{HCN}(4-3) / \mathrm{HCO}^{+}(4-3), \mathrm{R}_{\mathrm{HCN} / \mathrm{HCO}+}$}

In paper I, the $\mathrm{HCN}(4-3)$ and $\mathrm{HCO}^{+}(4-3)$ maps of VV 114 allowed us to investigate the central region at $200 \mathrm{pc}$ resolution for the first time, and we find that both the $\mathrm{HCN}(4-3)$ and $\mathrm{HCO}^{+}(4-3)$ in the eastern nucleus are compact $(<200 \mathrm{pc})$, and broad $\left[290 \mathrm{~km} \mathrm{~s}^{-1}\right.$ for $\mathrm{HCN}(4-3)]$. We present the $\mathrm{HCN}(4-3) / \mathrm{HCO}^{+}(4-$ $3), R_{\mathrm{HCN} / \mathrm{HCO}^{+}}$, image of VV 114 in Figure 8 . From the higher $R_{\mathrm{HCN} / \mathrm{HCO}}+$ along with the past X-ray and NIR observations, we suggest the presence of an obscured AGN in the eastern nucleus. We also detect a $3-4 \mathrm{kpc}$ long filament of dense gas, which is likely to be tracing the active star formation triggered by the ongoing merger, and this is consistent with the results from the numerical model by Teyssier et al. (2010) who predict that the fragmentation and turbulent motion of dense gas across the merging disk is responsible for forming dense gas clumps with masses of $10^{6}-10^{8} \mathrm{M}_{\odot}$.

We present the $R_{\mathrm{HCN} / \mathrm{HCO}}+$ image in Figure 8 . The overlap region does not show significant HCN (4-3) emis- sion, and we provide the $3 \sigma$ upper limit in Table 7 . Three out of the four boxes (i.e., S1 - S3) in the eastern nucleus have low $R_{\mathrm{HCN} / \mathrm{HCO}^{+}}(<0.5)$ whereas $\mathrm{S} 0$ has a high $R_{\mathrm{HCN} \mathrm{HCO}^{+}}(1.34 \pm 0.09)$. It is suggested that such a high value is only produced around AGN environments (e.g., Kohno et al. 2001; Harada et al. 2013; Iono et al. 2013; Izumi et al. 2013; Imanishi \& Nakanishi 2014).

\section{DERIVATION OF PHYSICAL PARAMETERS}

In this section, we derive the molecular gas mass ( $\$ 5.1)$, and the physical parameters using the radiative transfer code RADEX (§5.2) for each box defined in $\S 4$. The column density is derived using the optically thin ${ }^{13} \mathrm{CO}$ line under the LTE assumption. We estimate the beam filling factor $\Phi_{\mathrm{A}}$ and the relative molecular abundance of molecule $X$ (hereafter expressed as $\left.[X] /\left[\mathrm{H}_{2}\right]\right)(\S 5.3)$. Finally, we calculate the dust mass using the $340 \mathrm{GHz}$ continuum emission ( $\$ 5.4)$.

\subsection{Molecular Gas Mass Derivation}

The molecular gas mass $\mathrm{M}_{\mathrm{X}}$ is derived by;

$$
M_{\mathrm{X}}=\alpha_{\mathrm{X}} L_{\mathrm{X}}^{\prime}\left[\mathrm{M}_{\odot}\right],
$$

where $\alpha_{\mathrm{X}}$ is the molecular line luminosity-to- $\mathrm{H}_{2}$ mass conversion factor and $L^{\prime} \mathrm{X}$ is the velocity integrated flux (Solomon \& Vanden Bout 2005). We use the conversion factor known to be appropriate for $\mathrm{U} /$ LIRGs $\left(\alpha_{\mathrm{CO}}=0.8 \mathrm{M}_{\odot}\left(\mathrm{K} \mathrm{km} \mathrm{s}^{-1} \mathrm{pc}^{2}\right)^{-1}\right.$; Downes \& Solomon 1998). This is consistent with the value derived by Sliwa et al. (2013) in VV $114\left(\alpha_{\mathrm{CO}}=\right.$ $\left.0.5_{-0.3}^{+0.6} \mathrm{M}_{\odot}\left(\mathrm{K} \mathrm{km} \mathrm{s}^{-1} \mathrm{pc}^{2}\right)^{-1}\right)$. The molecular gas mass derived at the boxes defined in $\S 4$ ranges between 0.2 $\times 10^{8}\left(\frac{\alpha_{\mathrm{CO}}}{0.8}\right)$ and $4.8 \times 10^{8}\left(\frac{\alpha_{\mathrm{CO}}}{0.8}\right) \mathrm{M}_{\odot}$ (Table 10). We also calculate the dense gas mass $M_{\text {dense }}$ using $\alpha_{\mathrm{HCN}}=$ $10 \mathrm{M}_{\odot}\left(\mathrm{K} \mathrm{km} \mathrm{s}^{-1} \mathrm{pc}^{2}\right)^{-1}$ (Gao \& Solomon 2004) and the $\mathrm{HCN}(4-3)$ luminosity which is converted to the HCN (10 ) luminosity using $\mathrm{HCN}(4-3) / \mathrm{HCN}(1-0)=0.63$ (paper I; Imanishi et al. 2007). The dense gas mass ranges between $1.8 \times 10^{6}\left(\frac{\alpha_{\mathrm{HCN}}}{10}\right)$ and $3.8 \times 10^{7}\left(\frac{\alpha_{\mathrm{HCN}}}{10}\right) \mathrm{M}_{\odot}$ (Table 11).

We note that the $\mathrm{CO}$ luminosity-to- $\mathrm{H}_{2}$ mass conversion factor, $\alpha_{\mathrm{CO}}$, is very uncertain, and varies significantly 

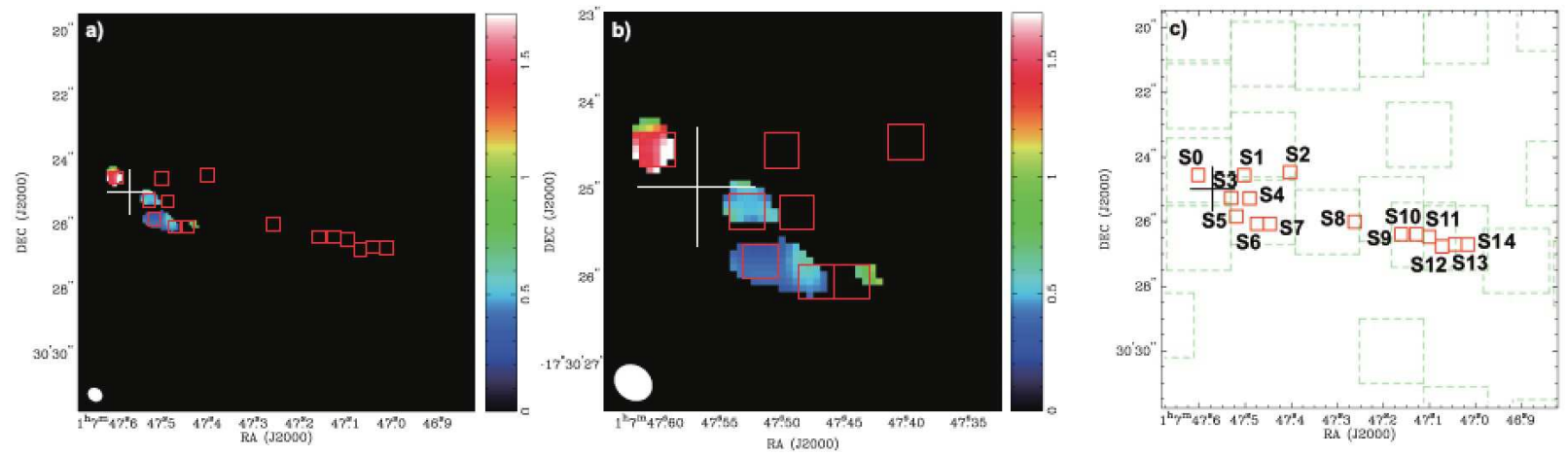

FIg. 8.- (a) The $R_{\mathrm{HCN} / \mathrm{HCO}}+$ image. The ratio in color scale ranges from 0 to 1 . The white cross shows the position of the eastern nucleus defined by the peak position of the Ks-band observation (Tateuchi et al. 2012). (b) The $R_{\mathrm{HCN} / \mathrm{HCO}+}$ image near the nucleus of VV 114E. The ratio in color scale also ranges from 0 to 1 . (c) Locations of 15 boxes (S0 - S14) that are used to calculate the line ratios and physical parameters. For each ratio, the two integrated intensity images were convolved to the same resolution and expressed in units of $\mathrm{K} \mathrm{km} \mathrm{s}^{-1}$ before calculating the ratio at locations where both lines are detected above $3 \sigma$. The black cross shows the position of the eastern nucleus defined by the peak position of the Ks-band observation (Tateuchi et al. 2012). The green open squares are the "R" boxes shown in Figure 7c.

from source to source $(0.4-0.8$ for LIRGs; Downes \& Solomon 1998; Yao et al. 2003; Papadopoulos et al. 2012; Bolatto et al. 2013). It may be possible that $\alpha_{\mathrm{CO}}$ varies from region to region within a galaxy. While one would ideally adopt a spatially varying $\alpha_{\mathrm{CO}}$ for a better quantification of the $\mathrm{H}_{2}$ mass, such a study is beyond the scope of this present paper. For simplicity, here we adopt a constant $\alpha_{\mathrm{CO}}$ across all regions in VV 114, bearing in mind that the uncertainties could be as large as a factor of two. The same applies to $\alpha_{\mathrm{HCN}}$ (Gao \& Solomon 2004)

\subsection{Radiative Transfer Analysis using RADEX}

We used the non-LTE radiative transfer code RADEX (van der Tak et al. 2007) and varied the parameters until the residuals between the observed line fluxes and the modeled line fluxes are minimized in a $\chi^{2}$ sense. We assumed a uniform spherical geometry $\left(d v=1.0 \mathrm{~km} \mathrm{~s}^{-1}\right)$, and derived the physical conditions of molecular gas $\left(T_{\text {kin }}, n_{\mathrm{H}_{2}}\right.$, and $\left.N\left(\mathrm{H}_{2}\right)\right)$. RADEX uses an escape probability approximation to solve the non-LTE excitation assuming that all lines are from the same region. Since the molecular lines in the band 7 have significantly higher critical densities than that in the band 3 , we used two sets of molecular lines; (case 1) 2.'0 box-summed ${ }^{12} \mathrm{CO}(1-$ $0),{ }^{13} \mathrm{CO}(1-0)$, and ${ }^{12} \mathrm{CO}(3-2)$, and (case 2) $1^{\prime \prime} .2$ boxsummed $\mathrm{HCN}(4-3), \mathrm{HCO}^{+}(4-3),{ }^{12} \mathrm{CO}(3-2)$, and ${ }^{12} \mathrm{CO}(1-0)$, to solve for the degeneracy of the physical parameters. In case 2 , we made the $u v$ and beammatched $\mathrm{HCN}(4-3), \mathrm{HCO}^{+}(4-3)$, and ${ }^{12} \mathrm{CO}(3-2)$ images $\left(1^{\prime \prime} .2 \times 1\right.$.'0 resolution with the P.A. = 119 deg. $)$, and we defined three $\mathrm{HCO}^{+}(4-3)$ peaks as E0, E1, and E2 (Figure 9). We also use the $u v$ and beam-matched ${ }^{12} \mathrm{CO}(1-0)$ data to constrain the $N\left(\mathrm{H}_{2}\right)$, allowing us to vary the $[\mathrm{HCN}] /\left[\mathrm{HCO}^{+}\right]$in case 2 . All line parameters, such as the upper state energies and the Einstein coefficients, were taken from the Leiden Atomic and Molecular Database (LAMDA; Schöier et al. 2005). In order to find the set of physical parameters that can reproduce the observed line intensities, we run RADEX by varying $T_{\text {kin }}, n_{\mathrm{H}_{2}}$, and $N\left(\mathrm{H}_{2}\right)$ for case 1 , and $T_{\text {kin }}, n_{\mathrm{H}_{2}}$, and $[\mathrm{HCN}] /\left[\mathrm{HCO}^{+}\right]$for case 2 . The adopted $N\left(\mathrm{H}_{2}\right)$ are $10^{21.2}, 10^{21.6}$, and $10^{21.5} \mathrm{~cm}^{-2}$, at E0, E1, and E2, re- spectively.

We varied the gas kinetic temperature within a range of $T_{\text {kin }}=5-300 \mathrm{~K}$ using steps of $\mathrm{d} T_{\text {kin }}=5 \mathrm{~K}$, and a gas density of $n_{\mathrm{H}_{2}}=10^{2}-10^{5} \mathrm{~cm}^{-3}$ using steps of $\mathrm{d} n_{\mathrm{H}_{2}}$ $=10^{0.1} \mathrm{~cm}^{-3}$. For case 1 , we fixed $\left[{ }^{13} \mathrm{CO}\right] /\left[\mathrm{H}_{2}\right]=1.4 \times$ $10^{-6}$ (Davis et al. 2013) and $[\mathrm{CO}] /\left[{ }^{13} \mathrm{CO}\right]=70$, which are the Galactic values (Wilson \& Rood 1994). In case 2 , we changed the parameters, $T_{\text {kin }}=5-400 \mathrm{~K}$ using steps of $\mathrm{d} T_{\text {kin }}=5 \mathrm{~K}, n_{\mathrm{H}_{2}}=10^{3}-10^{7} \mathrm{~cm}^{-3}$ using steps of $\mathrm{d} n_{\mathrm{H}_{2}}=10^{0.1} \mathrm{~cm}^{-3}$, and fixed $[\mathrm{CO}] /\left[\mathrm{H}_{2}\right]=1.0 \times 10^{-4}$ and $\left[\mathrm{HCO}^{+}\right] /\left[\mathrm{H}_{2}\right]=1.0 \times 10^{-9}$, which are the standard values observed in Galactic molecular clouds (Blake et al. 1987). We varied $[\mathrm{HCN}] /\left[\mathrm{HCO}^{+}\right]$from $1-10$, in steps of one. The parameters we used are summarized in Table 9. We list the results that are within the $95 \%$ confidence level with 3 -degree of freedom $\left(\chi^{2}<7.81\right)$ (Tables 12 and 13). Finally, we created velocity-averaged channel maps of $n_{\mathrm{H}_{2}}$ and the optical depth of the transitions (Figure 10).

We note that the uncertainty of the $N\left(\mathrm{H}_{2}\right)$ for case 2 did not strongly affect the results, while that of the $[\mathrm{CO}] /\left[{ }^{13} \mathrm{CO}\right]$ for case 1 changed. The effect of varying the $[\mathrm{CO}] /\left[{ }^{13} \mathrm{CO}\right]$ will be discussed in $\S 5.2 .1$. Future multitransition $\mathrm{HCN} / \mathrm{HCO}^{+} / \mathrm{CO} /{ }^{13} \mathrm{CO}$ imaging will help us to derive these parameters directly.

\subsubsection{Case 1}

The (box-averaged) kinetic temperature near the eastern nucleus (R21a) is constrained to within $25-90 \mathrm{~K}$ (the best fit is $50 \mathrm{~K})$, as shown in Table 12 . The $T_{\text {ex }}(58.8$ $\pm 2.9 \mathrm{~K}$ ) obtained from the LTE assumption at R21a (see $§ 5.3$ ) is higher than the best-fitted $T_{\text {kin }}$. In fact, we also find five regions (R10b, R11b, R14, R16, and R25a) that show similarly high excitation temperatures. Four out of five regions are in the central filament. In general, spontaneous emission dominates over collisional excitation in sub-thermally excited conditions, and hence $T_{\text {ex }}$ should be lower than $T_{\text {kin }}$. One reason for this discrepancy could be attributed to the incorrect assumption of $[\mathrm{CO}] /\left[{ }^{13} \mathrm{CO}\right]$. By varying this abundance ratio, we find that the temperature reversal (i.e. $T_{\text {kin }}>T_{\text {ex }}$ ) occurs only when $[\mathrm{CO}] /\left[{ }^{13} \mathrm{CO}\right]>150$. This is consistent with the results obtained by Sliwa et al. (2013) who used 

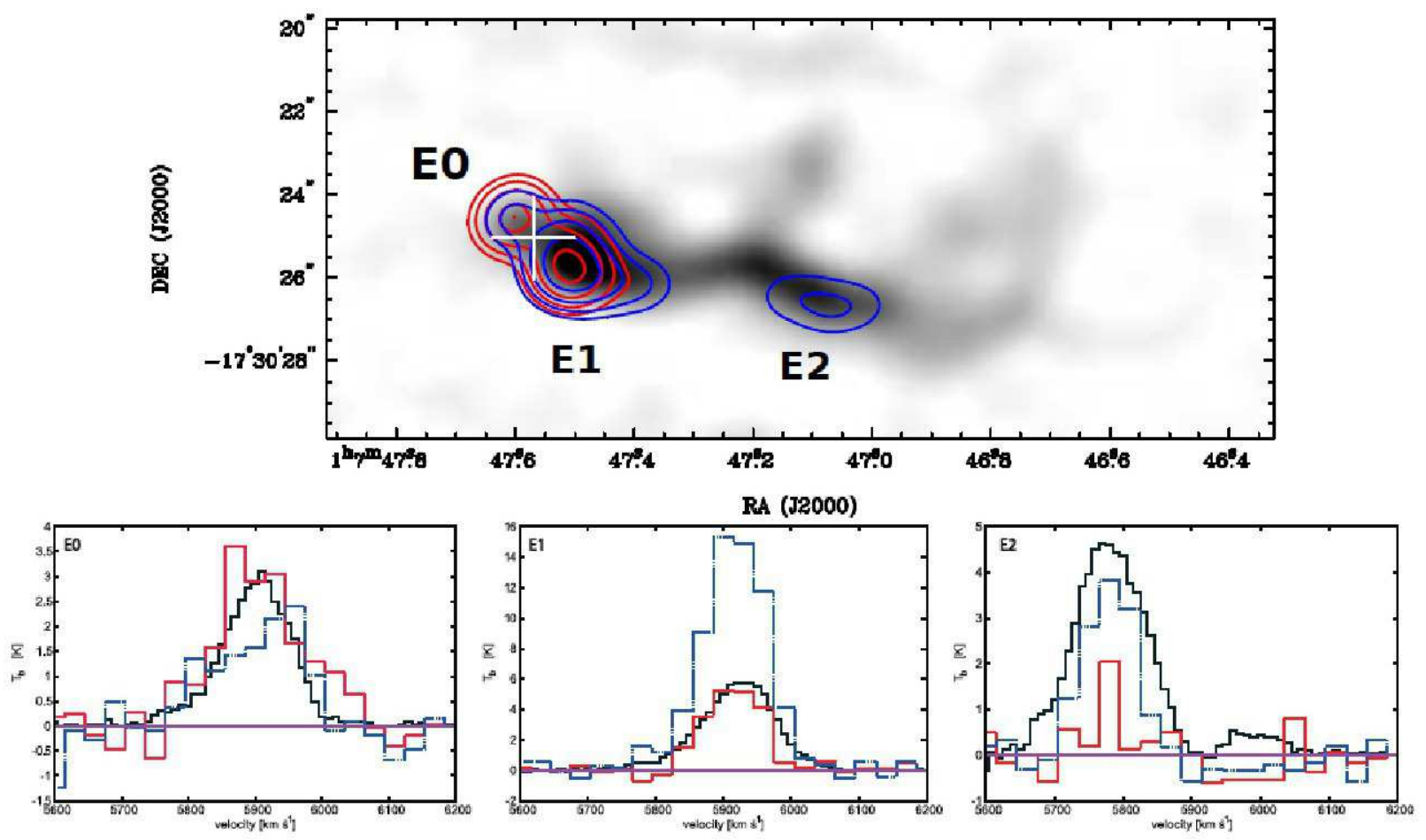

Fig. 9.- (top) The $u v$ - and beam-matched (grey color) ${ }^{12} \mathrm{CO}(3-2)$, (red contour) HCN (4-3), and (blue contour) $\mathrm{HCO}^{+}(4-3)$ images. The integrated intensity of the ${ }^{12} \mathrm{CO}(3-2)$ in color scale ranges from $0 \mathrm{Jy} \mathrm{km} \mathrm{s}{ }^{-1}$ to $100 \mathrm{Jy} \mathrm{km} \mathrm{s}^{-1}$. The contours are $5,10,20,40$, and $50 \mathrm{Jy} \mathrm{km} \mathrm{s}^{-1}$ for $\mathrm{HCN}(4-3)$, and $12,24,48$, and $96 \mathrm{Jy} \mathrm{km} \mathrm{s}^{-1}$ for $\mathrm{HCO}^{+}(4-3)$. The white cross shows the position of the eastern nucleus defined by the peak position of the Ks-band observation (Tateuchi et al. 2012). (bottom) 1.' 2 box-summed spectra of (black line) ${ }^{12} \mathrm{CO}(3-2)$, (red dashed line) $\mathrm{HCN}(4-3) \times 10$, and (blue dashed line) $\mathrm{HCO}^{+}(4-3) \times 10$ at the each box, labeled E0 - E2. The spectra are taken from the ALMA data cubes after correcting the cubes for the primary beam attenuation and convolving them to 1 !' $2 \times 1$.' 0 resolution (P.A. = 119 deg.)

RADEX along with their multi $\mathrm{CO}$ and ${ }^{13} \mathrm{CO}$ line data to find evidence of a cold/dense molecular gas component with extremely high $[\mathrm{CO}] /\left[{ }^{13} \mathrm{CO}\right]$ of 229 , which is 3 times higher than that of the Galactic value (Wilson \& Rood 1994).

The derived $T_{\text {kin }}$ at the other regions are generally higher than $100 \mathrm{~K}$. The derived $T_{\mathrm{ex}}$ in each region are typically $10-40 \mathrm{~K}$, which may suggest sub-thermal conditions. The kinetic temperatures derived at the SE and NW arms are estimated to be $<90 \mathrm{~K}$, with higher temperature at the NW arm. The NW arm is also associated with relatively strong $\mathrm{Pa} \alpha$ emission and $K$ s-band emission, which is consistent with the higher relative temperature due to star-forming activities (Minamidani et al. 2008). However, this is inconsistent with the general understanding that strong tidal shear in tidal arms prevents active star formation to occur (Aalto et al. 2010).

The derived $n_{\mathrm{H}_{2}}$ in most of the boxes are less than $10^{3.0} \mathrm{~cm}^{-3}$, which is consistent with the critical densities of the low- $J$ CO lines observed here. The highest density of $10^{3.4}-10^{5.0} \mathrm{~cm}^{-3}$ is estimated at R21a, and this is consistent with the location of the eastern nucleus. Since we also observed the strongest HCN (4-3) and $\mathrm{HCO}^{+}(4-3)$ emission at R21a at the same line-ofsight velocity (Iono et al. 2013, see also Appendix A.3), it is possible that the main contribution to the $\mathrm{CO}$ emission at R21a arises from dense gas $\left(10^{3.4}-10^{5.0} \mathrm{~cm}^{-3}\right)$ near the eastern nucleus, with a minor contribution from the diffuse gas clouds along the same line of sight observed within the same beam. In contrast to the eastern nucleus, the boxes that cover the western galaxy (R1 R11 and R26 - R29) show moderately dense condition of $10^{2.0}-10^{4.0} \mathrm{~cm}^{-3}$. This extended and moderately dense gas is associated with the disk-like structure seen in optical images (Evans 2008), and the star formation traced in $\mathrm{Pa} \alpha$ emission and UV/X-ray emission (Grimes et al. 2006; Tateuchi et al. 2012). We note that the strongest off-nuclear Pa $\alpha$ peak (R27 in Table 10; SFR $=3.15 \pm$ $0.05 \mathrm{M}_{\odot} \mathrm{yr}^{-1}$ ) coincides with relatively low gas density $\left(\sim 10^{3.0} \mathrm{~cm}^{-3}\right)$. The density of the surrounding region labeled R25a is similar $\left(10^{3.5}-10^{5.0} \mathrm{~cm}^{-3}\right)$ and this is comparable to the nucleus of the eastern galaxy. The secondary $\mathrm{Pa} \alpha$ peak $\left(\mathrm{R} 29 ; \mathrm{SFR}=0.92 \pm 0.05 \mathrm{M}_{\odot} \mathrm{yr}^{-1}\right)$ is not associated with any molecular line emission.

It is usually believed that the ${ }^{12} \mathrm{CO}(1-0)$ emission is optically thick $\left(\tau_{\mathrm{CO}} \gg 1\right)$, while the ${ }^{13} \mathrm{CO}(1-0)$ emission is optically thin $\left(\tau_{13} \mathrm{CO} \ll 1\right)$ even in luminous mergers (Davis et al. 2013). In most regions, we find that the optical depth of the ${ }^{12} \mathrm{CO}(1-0)$ line is $\gg 1$ (Figure 10). In contrast, the ${ }^{12} \mathrm{CO}(1-0)$ opacity at the eastern nucleus and the filament is moderately optically thick $\left(\tau_{\mathrm{CO}} \sim 1\right)$. However, the elevated $R_{12 / 13}$ at the eastern nucleus (see $\S 4)$ cannot be explained by the relatively low ${ }^{12} \mathrm{CO}(1-0)$ opacity alone (the opacity has to be $\tau_{\mathrm{CO}} \ll 0.1$; see also Wilson et al. (2009)). Finally, we find that indeed the ${ }^{13} \mathrm{CO}(1-0)$ emission is optically thin $\left(\tau_{13} \mathrm{CO} \ll 1\right)$ averaged over the whole galaxy, except for the southern dust lane $\left(\tau_{13} \mathrm{CO}=0.3-1.5\right)$.

From these results, we suggest that the peak of the 

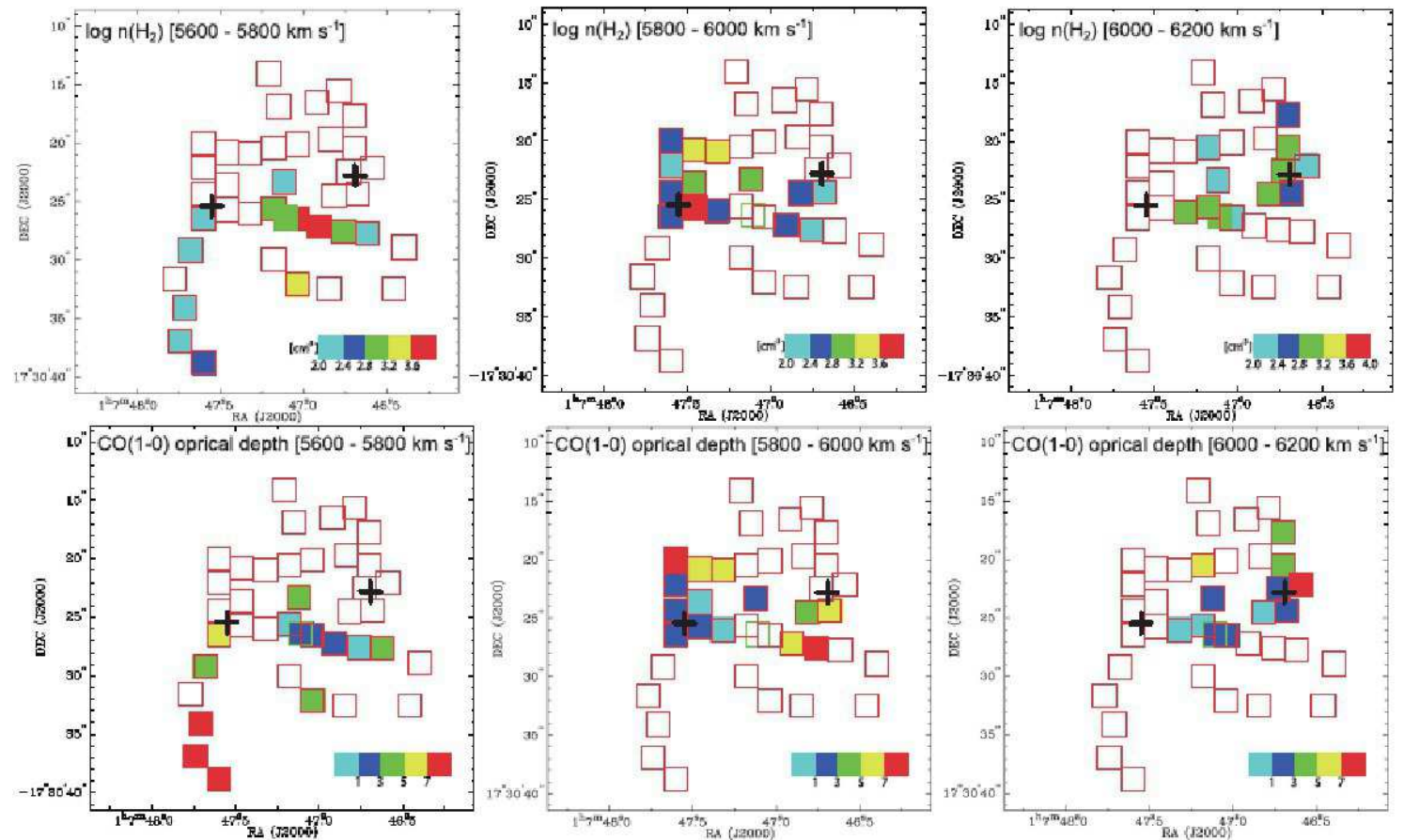

FIG. 10. - The channel maps of the box-averaged RADEX modeling based on the ${ }^{12} \mathrm{CO}(1-0),{ }^{13} \mathrm{CO}(1-0)$, and ${ }^{12} \mathrm{CO}(3-2)$. (top) The best fitted values of logarithmic gas density of the ${ }^{12} \mathrm{CO}(1-0)$ emission. The value in color scale ranges from 2.0 to $4.0 \mathrm{~cm}{ }^{-3}$. (bottom) The best fitted values of optical depth of the ${ }^{12} \mathrm{CO}(1-0)$ emission. The value in color scale ranges from 0 to 9 . The black crosses show the positions of the nuclei defined by the peak positions of the Ks-band observation (Tateuchi et al. 2012). The open squares are regions which we cannot solve the RADEX calculations because of non-detection of the ${ }^{12} \mathrm{CO}(1-0),{ }^{13} \mathrm{CO}(1-0)$, or ${ }^{12} \mathrm{CO}(3-2)$ emission.

molecular gas in the central $800 \mathrm{pc}$ of the eastern galaxy is cold $\left(T_{\text {kin }}=25-90 \mathrm{~K}\right)$, dense $\left(n_{\mathrm{H}_{2}}=10^{3.4}-\right.$ $\left.10^{5.0} \mathrm{~cm}^{-3}\right)$, and moderately optically thick $\left(\tau_{\mathrm{CO}(1-0)}\right.$ $\sim 3)$, while peaks in the overlap region are warm $\left(T_{\text {kin }}\right.$ $>50 \mathrm{~K}$, best-fitted $T_{\text {kin }}$ is 95 and $175 \mathrm{~K}$ at R39a and $\mathrm{R} 39 \mathrm{~b}$, respectively), moderately dense $\left(n_{\mathrm{H}_{2}}=10^{2.3}-\right.$ $\left.10^{4.1} \mathrm{~cm}^{-3}\right)$, and moderately optically thick $\left(\tau_{\mathrm{CO}(1-0)} \sim\right.$ $1)$. The derived density of the eastern galaxy is slightly higher than the range of values found in U/LIRGs using low- $J$ CO emission with $\sim \mathrm{kpc}$ resolution $\left(n_{\mathrm{H}_{2}}=10^{2.3}-\right.$ $10^{4.3} \mathrm{~cm}^{-3}$; Downes \& Solomon 1998). In addition, the low opacities predicted from these analyses are consistent with earlier results that investigate the opacities in M82 $(\tau=0.5-4.5$; Mao et al. 2000) and U/LIRGs $(\tau=3-$ 10; Downes \& Solomon 1998), and the central region of NGC $6240(\tau=0.2-2$; Iono et al. 2007). However, the derived temperature of the eastern galaxy is inconsistent with the high values found in nearby starburst galaxies M82, NGC 253, and NGC 6240 (Wild et al. 1992; Jackson et al. 1995; Seaquist \& Frayer 2000; Iono et al. 2007). The disagreement is possibly due to the uncertainties in the $[\mathrm{CO}] /\left[{ }^{13} \mathrm{CO}\right]$, or the difference in the observed molecular gas tracers.

\subsubsection{Case 2}

The values for $T_{\text {kin }}, n_{\mathrm{H}_{2}}$, and the optical depth of HCN (4-3) and $\mathrm{HCO}^{+}(4-3)$ are shown in Table 8. The derived parameters for the unresolved component E0, are $T_{\text {kin }}>100 \mathrm{~K}, n_{\mathrm{H}_{2}}=10^{5.0}-10^{5.4} \mathrm{~cm}^{-3}$, and $[\mathrm{HCN}] /\left[\mathrm{HCO}^{+}\right]>5$. The lower limit to the kinetic temperature is higher than those of E1 and E2, mainly due to the unusually high $R_{\mathrm{HCN} / \mathrm{HCO}^{+}}$and $R_{\mathrm{HCN} / \mathrm{CO}}$. In contrast to E0, the derived parameters near E1 show high $\mathrm{H}_{2}$ densities $\left(n_{\mathrm{H}_{2}}=10^{5.6}-10^{5.9} \mathrm{~cm}^{-3}\right)$. The overlap region $(\mathrm{E} 2)$, where the star-formation rate $(1.70 \pm$ $\left.0.05 \mathrm{M}_{\odot} \mathrm{yr}^{-1}\right)$ is lower than the eastern nucleus, has densities in the range of $n_{\mathrm{H}_{2}}=10^{5.0}-10^{5.6} \mathrm{~cm}^{-3}$. Finally, the optical depths for the $\mathrm{HCO}^{+}(4-3)$ and $\mathrm{HCN}(4-3)$ lines are calculated for each gas clump, yielding $\tau_{\mathrm{HCN}} \simeq$ 0.7 and $\tau_{\mathrm{HCO}+} \simeq 0.2$ for $\mathrm{E} 0, \tau_{\mathrm{HCN}} \simeq 0.2$ and $\tau_{\mathrm{HCO}^{+}} \simeq$ 0.6 for $\mathrm{E} 1$, and $\tau_{\mathrm{HCN}} \simeq 0.4$ and $\tau_{\mathrm{HCO}^{+}} \simeq 0.4$ for $\mathrm{E} 2$.

The higher linear resolution observations of HCN (43 ) and $\mathrm{HCO}^{+}$(4-3) toward NGC 1097 (Izumi et al. 2013) revealed that the gas in the central region of NGC 1097 has $T_{\text {kin }}=70-550 \mathrm{~K}$ and $n_{\mathrm{H}_{2}}=10^{4.5}-$ $10^{6.0} \mathrm{~cm}^{-3}$. Moreover, by comparing to LVG models, Krips et al. (2008) found that $\mathrm{HCN}$ and $\mathrm{HCO}^{+}$emission in AGN-dominated sources appears to emerge from regions with lower $\mathrm{H}_{2}$ densities, higher temperatures, and higher HCN abundance relative to starburst-dominated (SB-dominated) galaxies. Our results obtained toward VV 114 are consistent with these previous results.

\subsection{Filling factor and Column Density under LTE}

In order to determine the bulk properties of the $\mathrm{CO}$ emitting gas, we used an excitation temperature analysis (Davis et al. 2013). The excitation temperature at each box can be calculated from

$T_{\mathrm{ex}}=T_{0}\left(\ln \left[\left(\frac{T_{\mathrm{b}, \mathrm{CO}(1-0)}}{\Phi_{\mathrm{A}} T_{0}\left(1-e^{\left.-\tau_{\mathrm{CO}}\right)}\right.}+\frac{1}{e^{T_{0} / T_{\mathrm{b} g}}-1}\right)^{-1}+1\right]\right)^{-1}$ 
where $T_{0}=h \nu / k$ [ $=5.53 \mathrm{~K}$ for ${ }^{12} \mathrm{CO}(1-0)$ emission], $\nu$ is the frequency of the transition, $h$ is the Planck's constant, $k$ is the Boltzmann's constant, $T_{\mathrm{b}, \mathrm{CO}(1-0)}$ is the brightness temperature of ${ }^{12} \mathrm{CO}(1-0)$ emission in Kelvin, $\tau_{\mathrm{CO}}$ is the optical depth of the ${ }^{12} \mathrm{CO}(1-0)$ emission, and $T_{\mathrm{bg}}$ is the cosmic microwave background temperature $(2.73 \mathrm{~K})$. Using $T_{\text {kin }}$ estimated from the RADEX calculation ( $\$ 5.2)$, we estimate the beam filling factor $\Phi_{\mathrm{A}}$,

$$
\Phi_{\mathrm{A}}=\frac{T_{\mathrm{b}, \mathrm{CO}(1-0)}}{T_{\mathrm{kin}}}
$$

The optical depth of the ${ }^{12} \mathrm{CO}(1-0)$ emission is also estimated from the RADEX calculation in $§ 5.2$. Assuming that the ${ }^{13} \mathrm{CO}$ and $\mathrm{CO}$ arise from the same molecular cloud, and that the ${ }^{12} \mathrm{CO}(1-0)$ is optically thick, we estimate the optical depth of a given molecule using,

$$
\tau_{\mathrm{X}} \simeq \ln \left[\left(1-\frac{T_{\mathrm{b}, \mathrm{X}}}{T_{\mathrm{b}, \mathrm{CO}(1-0)}}\right)^{-1}\right]
$$

where $\tau_{\mathrm{X}}$ is the optical depth of a given transition, and $T_{\mathrm{b}, \mathrm{X}}$ is the observed brightness temperature for transition X. Using $T_{\text {ex }}$ and $\tau_{\mathrm{X}}$, we estimate the column density for a given molecule from,

$$
\begin{gathered}
N_{\mathrm{X}}=\frac{3 k}{8 \pi^{3} \mu^{2} B(J+1)} \frac{\exp \left(\frac{2 h J(J+1)}{k T_{\mathrm{ex}}}\right)}{\left(1-\exp \left(-\frac{h \nu}{k T_{\mathrm{ex}}}\right)\right)} \\
\times \frac{\tau_{\mathrm{X}}}{1-e^{-\tau_{\mathrm{X}}}} \frac{1}{J\left(T_{\mathrm{ex}}\right)-J\left(T_{\mathrm{bg}}\right)} \int T_{\mathrm{R}}^{*} d V \\
J(T)=\frac{h \nu}{k} \frac{1}{\exp (h \nu / k T)-1}
\end{gathered}
$$

where $\mu$ is the dipole moment, $B$ is the rotational constant, $J$ is the lower energy level, and $\int T_{\mathrm{R}}^{*} d V$ is the integrated intensity (Wilson et al. 2009). The derived column densities are listed in Tables 14 and 15.

\subsection{Dust Mass and ISM Mass Derivation from $340 \mathrm{GHz}$ continuum}

We calculated the dust mass from the $340 \mathrm{GHz}$ $(880 \mu \mathrm{m})$ continuum emission (Table 3) using (Wilson et al. 2008),

$$
M_{\text {dust }}=74220 S_{340} D_{\mathrm{L}}^{2} \frac{e^{\frac{17}{T_{d}}}-1}{\kappa_{340}} \mathrm{M}_{\odot}
$$

where $S_{340}$ is the $340 \mathrm{GHz}$ flux in Jy and $D_{\mathrm{L}}$ is the luminosity distance in Mpc. We assumed a dust emissivity, $\kappa_{340}=0.9 \mathrm{~cm}^{2} \mathrm{~g}^{-1}$, and the dust temperature $T_{\mathrm{d}}$ of $39.4 \mathrm{~K}$ (Wilson et al. 2008). The box-summed dust masses ranges between $2.0 \times 10^{4}\left(\frac{0.9}{\kappa_{340}}\right)$ and $2.8 \times$ $10^{6}\left(\frac{0.9}{\kappa_{340}}\right) \mathrm{M}_{\odot}($ Table 10$)$. We note that we used the Draine \& Lee (1984) dust model for $\kappa_{340}$, because the $\kappa_{340}$ derived from observations has a large error (Henning et al. 1995).

Scoville et al. (2014) suggested that the submillimeter continuum emission traces the total ISM mass $\left(M_{\mathrm{ISM}}\right)$, since the long wavelength Rayleigh-Jeans (RJ) tail of thermal dust emission is often optically thin. In order to compare the $M_{\text {ISM }}$ with the $M_{\mathrm{H}_{2}}$ (see $\$ 5.1$ ) using spatially-resolved data, we calculated the total ISM mass from the $340 \mathrm{GHz}$ continuum emission (Scoville et al. 2014). For $\nu_{\text {rest }} \lesssim 1199 \mathrm{GHz}$,

$$
\begin{aligned}
S_{\nu_{\mathrm{obs}}}=0.83 & \frac{M_{\mathrm{ISM}}}{10^{10} \mathrm{M}_{\odot}}(1+z)^{4.8}\left(\frac{\nu_{\mathrm{obs}}}{353 \mathrm{GHz}}\right)^{3.8} \\
& \times \frac{\Gamma_{\mathrm{RJ}}}{\Gamma_{0}}\left(\frac{\mathrm{Gpc}}{D_{\mathrm{L}}}\right) \text { mJy }
\end{aligned}
$$

where $S_{\nu_{\mathrm{obs}}}$ is the observed flux, $M_{\mathrm{ISM}}$ is the ISM mass, $\nu_{\mathrm{obs}}$ is the observed frequency, and $\Gamma_{\mathrm{RJ}}$ and $\Gamma_{0}$ are given by

$$
\begin{gathered}
\Gamma_{\mathrm{RJ}}\left(T_{d}, \nu_{\mathrm{obs}}, z\right)=\frac{h \nu_{\mathrm{obs}}(1+z) / k T_{d}}{e^{h \nu_{\mathrm{obs}}(1+z) / k T_{d}}-1} \\
\Gamma_{0}=\Gamma_{\mathrm{RJ}}\left(T_{d}, 353 \mathrm{GHz}, 0\right) .
\end{gathered}
$$

The derived box-summed ISM masses of VV 114 range between $5.2 \times 10^{7}$ and $7.2 \times 10^{8} \mathrm{M}_{\odot}$ (Table 10). This is comparable to the box-summed $\mathrm{H}_{2}$ masses $\left(M_{\mathrm{H}_{2}}=(0.2\right.$ $\left.-4.7) \times 10^{8}\left(\frac{\alpha_{\mathrm{CO}}}{0.8}\right) \mathrm{M}_{\odot}\right)$. We find that the $M_{\mathrm{ISM}} / M_{\mathrm{H}_{2}}$ ratio is close to unity $\left(0.5-2.0\right.$, the average $M_{\mathrm{ISM}} / M_{\mathrm{H}_{2}}$ $=0.9 \pm 0.1$, while the total $M_{\mathrm{ISM}} / M_{\mathrm{H}_{2}}$ ratio is $0.6 \pm$ 0.1 . This means that the spatially-resolved $M_{\text {ISM }}$ is a good tracer of the "resolved" $\mathrm{H}_{2}$ mass. However, the total $M_{\text {ISM }}$ underestimates the $\mathrm{H}_{2}$ mass (even using the $\alpha_{\mathrm{CO}}$ for ULIRGs to derive the $M_{\mathrm{H}_{2}}$ ) because the global distribution of the $340 \mathrm{GHz}$ continuum emission is significantly different from that of the CO (1-0) emission (Figures 2 and 6). This difference between the $340 \mathrm{GHz}$ continuum and the $\mathrm{CO}(1-0)$ is also seen in recent observations of nearby LIRGs (e.g., Sakamoto et al. 2014).

\section{DISCUSSION}

\subsection{Conditions of "Dense" Gas near the Eastern Nucleus}

Our RADEX modeling yields lower molecular gas density near the AGN $\left(n_{\mathrm{H}_{2}}=10^{5.0}-10^{5.4} \mathrm{~cm}^{-3}\right)$ compared to the surrounding clumps $\left(10^{5.6}-10^{5.9} \mathrm{~cm}^{-3}\right)$. Similarly high values are obtained near AGNs in other galaxies (Alonso-Herrero et al. 2002; Wilson et al. 2003; Krips et al. 2008). Krips et al. (2008) suggest that the gas densities in AGN host galaxies $\left(<10^{4.5} \mathrm{~cm}^{-3}\right)$ are lower than starburst host galaxies $\left(10^{5.0}-10^{6.5} \mathrm{~cm}^{-3}\right)$, and a common interpretation relies on a clumpy ISM near starforming regions (which reduces the filling factor) and a continuous ISM near the AGN. Since our current observations $(\sim 200 \mathrm{pc}$ resolution) cover a significantly large area and the beam filling factor may be small $\left(\Phi_{\mathrm{A}}\right.$ at E0, E1, and E2 are $\lesssim 0.03,0.04-0.06$ and $0.01-0.04$, respectively), higher resolution observations $(<0.5)$ are required to confirm this scenario.

In addition, our modeling shows higher $[\mathrm{HCN}] /\left[\mathrm{HCO}^{+}\right]$ near the eastern nucleus $(>5)$ than that in the surrounding clumps $(<4)$ and the overlap region $(1-9)$. The elevated $[\mathrm{HCN}] /\left[\mathrm{HCO}^{+}\right]$is explained by two mechanisms (Krips et al. 2008). One is far-UV radiation from OB stars in young starbursts (Sternberg \& Dalgarno 1995), and the other is strong X-ray radiation from an AGN 

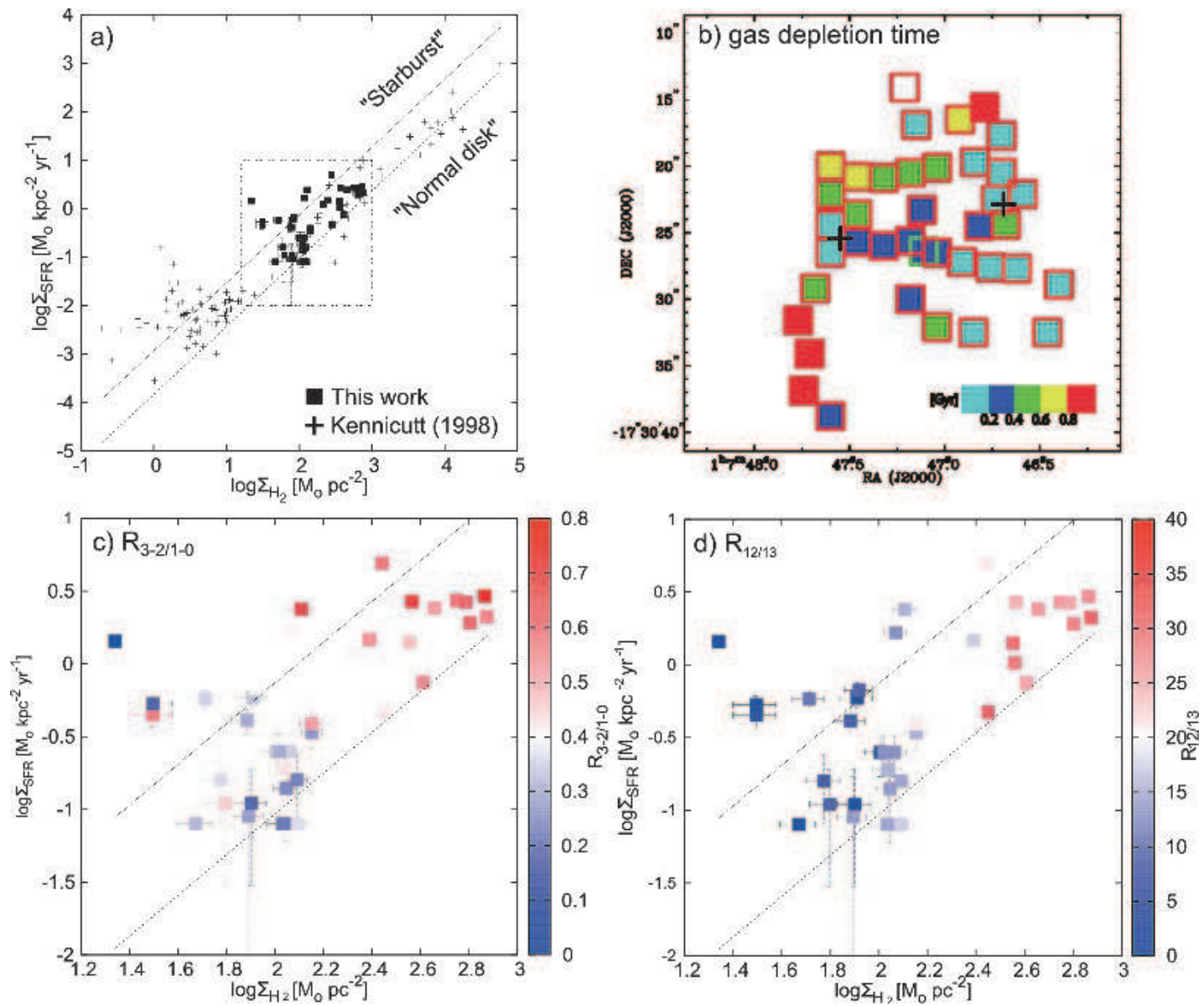

FIG. 11.- (a) The Kennicutt-Scimidt law of VV 114 overlaid on other galaxies. Filled squares show regions of VV 114, while crosses show galaxies in the sample of Kennicutt (1998). The dashed line and the dotted line indicate the "starburst" sequence and "normal disk" sequence, respectively (Daddi et al. 2010). (b) Distribution of the box-averaged gas depletion time $\left(\tau_{\text {gas }}=\Sigma_{\mathrm{H}_{2}} / \Sigma_{\mathrm{SFR}}\right)$. The gas depletion time in color scale ranges from $0-1$ Gyr. The black crosses show the positions of the nuclei defined by the peak positions of the Ks-band observation (Tateuchi et al. 2012). The open square is a region which we cannot estimate the gas depletion time because of non-detection of the Pao emission (Tateuchi et al. 2012). (c) The Keniccutt-Schmidt law with the $R_{3-2 / 1-0}$. The ratio in color scale ranges from 0 to 0.8. (d) The Keniccutt-Schmidt law with the $R_{12 / 13}$. The ratio in color scale ranges from 0 to 40 .

(Maloney et al. 1996). Because of different penetrating lengths between far-UV and X-ray emission, photon dominated regions (PDRs) are created at the surface of gas clouds and X-rays penetrate deeply into the circumnuclear disk (CND), forming large X-ray dominated regions. As a consequence of this volume versus surface effect, the X-ray radiation from an AGN may produce higher $\mathrm{HCN}$ abundances than the UV radiation of starburst activities (Krips et al. 2008). To some degree, ionization effects from cosmic rays (Wild et al. 1992) such as supernovae or strong shocks are suspected to significantly increase the $\mathrm{HCO}^{+}$abundance while potentially decreasing the HCN abundance, thus yielding lower $R_{\mathrm{HCN} / \mathrm{HCO}^{+}}$in evolved starbursts than in AGNs. The high $[\mathrm{HCN}] /\left[\mathrm{HCO}^{+}\right]$near the eastern nucleus and low $[\mathrm{HCN}] /\left[\mathrm{HCO}^{+}\right]$and strong/extended $8 \mathrm{GHz}$ continuum detection at the surrounding clumps (Condon et al. 1991) are all consistent with a presence of an AGN in the eastern nucleus, surrounded by star-forming dense clumps.

\subsection{Spatially Resolved Kennicutt-Schmidt Law}

Observational studies of galaxies at global scales have shown that the surface density of SFR and that of cold gas traced in $\mathrm{CO}(1-0)$ obey a power law relation (KS law; Schmidt 1959; Kennicutt 1998). ULIRGs are systemically shifted from the normal galaxy population in the $\Sigma_{\mathrm{SFR}}-\Sigma_{\mathrm{H}_{2}}$ phase (Komugi et al. 2005; Daddi et al. 2010; Genzel et al. 2010; Leroy et al. 2013). It is suggested that systems lower in IR luminosity (e.g., LIRGs) occupy the region between the "starburst" sequence and the "normal disk" sequence in the KS law. Galaxies in the "starburst" sequence have shorter gas depletion time $\left(\tau_{\text {gas }}=\Sigma_{\mathrm{H}_{2}} / \Sigma_{\mathrm{SFR}} \sim 0.1\right.$ Gyr $)$ relative to galaxies in the "normal disk" sequence $\left(\tau_{\text {gas }} \sim 1\right.$ Gyr; Daddi et al. 2010; Bournaud et al. 2011). The spatially resolved surface densities of the SFR and the molecular gas mass of VV 114 are shown in Table 10 and Figure 11. The starforming regions of VV 114 fill the gap between the "normal disk" and "starburst" sequences (Figure 11a). We also show the spatial distribution of $\tau_{\text {gas }}$ in Figure $11 \mathrm{~b}$. The data points close to the "starburst" sequence are located along the eastern nucleus $\left(<0.2\left(\frac{\alpha_{\mathrm{CO}}}{0.8}\right)\right.$ Gyr $)$ and the overlap region $\left(=0.2-0.4\left(\frac{\alpha_{\mathrm{CO}}}{0.8}\right) \mathrm{Gyr}\right)$, while those near the "normal disk" sequence are located in the NW 
and SE arms $\left(>0.8\left(\frac{\alpha_{C O}}{0.8}\right)\right.$ Gyr $)$. The spatial distribution of $\Sigma_{\mathrm{SFR}}$ and $\Sigma_{\mathrm{H}_{2}}$ are consistent with the distributions of previous optical, UV, and X-ray studies (Alonso-Herrero et al. 2002; Le Floc'h et al. 2002; Grimes et al. 2006). Regions with higher $\Sigma_{\mathrm{SFR}}$ and $\Sigma_{\mathrm{H}_{2}}$ clearly show higher $R_{3-2 / 1-0}$ and $R_{12 / 13}$ (Figures $11 \mathrm{c}$ and $11 \mathrm{~d}$ ).

In summary, transition from the "normal disk" to "starburst" sequence may occur when the molecular clouds become excited and dense at the nuclei and the overlap region. Moreover, gas clouds with high $R_{3-2 / 1-0}$ have high $\Sigma_{\mathrm{SFR}}-\Sigma_{\mathrm{H}_{2}}$, and this is consistent with past studies which suggest that the $R_{3-2 / 1-0}$ correlates with the local $\mathrm{H} \alpha$ flux (Minamidani et al. 2008; Fujii et al. 2014). The $R_{12 / 13}$ also shows a similar trend, and this is also consistent with the past studies $(>20$ in central kpc regions of U/LIRGs, $10-15$ in normal starburst galaxies, and $\sim 5$ in Galactic GMCs; Aalto et al. 1997): The reason for the elevated $R_{12 / 13}$ in starburst regions of VV 114 will be discussed in detail in $\$ 6.3$.

\subsection{CO Isotope Ratio Enhancement in the Molecular "Filament"}

We suggest from our RADEX modelings that the eastern nucleus and the overlap region have extremely high $[\mathrm{CO}] /\left[{ }^{13} \mathrm{CO}\right](>200)$, which is at least two times higher than the Galactic value ( $\simeq 70$; Wilson \& Rood 1994). The $\mathrm{Pa} \alpha$ peaks roughly coincide with the regions where high $[\mathrm{CO}] /\left[{ }^{13} \mathrm{CO}\right]$ are expected, suggesting that the increased $[\mathrm{CO}] /\left[{ }^{13} \mathrm{CO}\right]$ is related to the star formation activity. Similarly high values are seen in the overlap region of NGC4038/9 (Wilson et al. 2003) and the Taffy (Zhu et al. 2007). Zhu et al. (2007) suggested that the extreme $[\mathrm{CO}] /\left[{ }^{13} \mathrm{CO}\right]$ value in the bridge is explained by three scenarios, 1) selective isotope photodissociation in the diffuse clouds and shocked region, 2) $\mathrm{CO}$ enrichment around starburst activities, and/or 3) the destruction and recombination of molecules after shock. We briefly explain each scenario below, but our current data is insufficient for us to identify the exact cause of the high $[\mathrm{CO}] /\left[{ }^{13} \mathrm{CO}\right]$ in $\mathrm{VV} 114$.

The first possibility of $[\mathrm{CO}] /\left[{ }^{13} \mathrm{CO}\right]$ enhancement is the deficiency in ${ }^{13} \mathrm{CO}$. Sheffer et al. (1992) suggest that selective isotope photodissociation can reduce the ${ }^{13} \mathrm{CO}$ abundance in diffuse clouds, because $\mathrm{CO}$ is selfshielded to a greater extent. Thus, the ISM surrounding young starbursts and/or shocked regions show elevated $[\mathrm{CO}] /\left[{ }^{13} \mathrm{CO}\right]$ (Zhu et al. 2007). The ISM in the nuclei and the overlap region of VV 114 show extremely high $[\mathrm{CO}] /\left[{ }^{13} \mathrm{CO}\right]$, presumably due to intense starburst activities and/or large-scale shocks.

The second possibility is that massive stars end their life as supernovae and expel a large amount of ${ }^{12} \mathrm{C}$ in the interstellar medium. While the elemental abundances (e.g. C and S) are not directly related to the molecular abundances (e.g., CS; Casoli et al. 1992), once the synthesized elements are dispersed in the interstellar medium, molecules (e.g., CO, CS, and CN; Henkel et al. 2014) form as soon as the temperature and density conditions are favorable. This occurs with a timescale of a few $10^{5}$ yr (Langer \& Graedel 1989).

For the overlap region, the destruction and recombination of molecules after shocks (see §6.5) are possible mechanisms to enhance the $[\mathrm{CO}] /\left[{ }^{13} \mathrm{CO}\right]$ (the third pos-

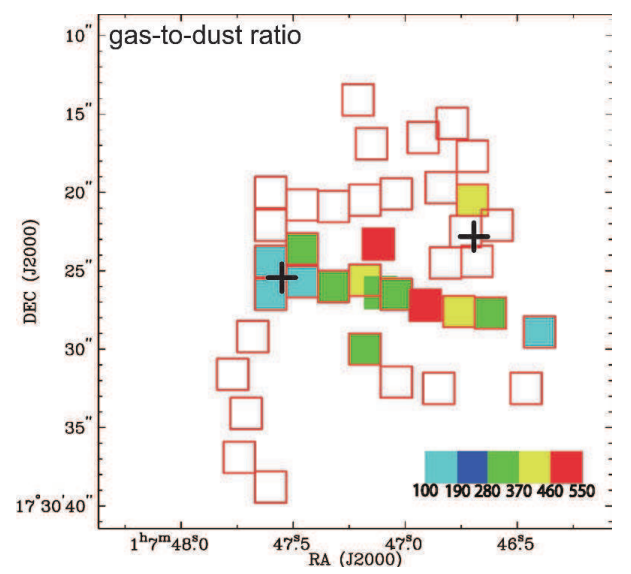

FIG. 12.- Distribution of the box-averaged gas-to-dust ratio map. The black crosses show the positions of the nuclei defined by the peak positions of the Ks-band observation (Tateuchi et al. 2012). The open squares are regions which we cannot estimate the gas-to-dust ratio because of non-detection of the $340 \mathrm{GHz}$ continuum emission.

sibility). The recombination timescale of $\mathrm{H}_{2}$ and $\mathrm{CO}$ molecules after shock destruction are shorter than that of ${ }^{13} \mathrm{CO}$, since ionized photons from shocked regions lead to selective isotope photodissociation (Zhu et al. 2003). Shielded regions from the radiation field are needed to form rare ${ }^{13} \mathrm{CO}$ (Abundant $\mathrm{CO}$ can form self-shielded regions). Moreover, the rare isotope molecules generally need a longer time to form, because collisions between molecules and dust grains are less frequent (Zhu et al. 2007).

\subsection{Gas-to-Dust Ratio, $M_{\mathrm{H}_{2}} / M_{\text {dust }}$}

The gas-to-dust ratio, $M_{\mathrm{H}_{2}} / M_{\text {dust }}$, provides an important measure of the relative abundance between gas and metallicity. The average $M_{\mathrm{H}_{2}} / M_{\text {dust }}$ over the entire galaxy is often derived in single-dish work, and typical $M_{\mathrm{H}_{2}} / M_{\text {dust }}$ is $200-300$ for local U/LIRGs (Contini \& Contini 2003; Yao et al. 2003; Seaquist et al. 2004), and $15-231$ in high-z sources (Solomon \& Vanden Bout 2005). Wilson et al. (2008) found $M_{\mathrm{H}_{2}} / M_{\text {dust }}=357 \pm$ 95 from a sample of 13 U/LIRGs, including VV 114, observed at kpc resolution.

We use the gas and dust masses derived in $\S 5.4$ to investigate the distribution of $M_{\mathrm{H}_{2}} / M_{\text {dust }}$ (Figure 12). The smallest value of $(128 \pm 16)\left(\frac{\alpha_{C O}}{0.8}\right)\left(\frac{0.9}{\kappa_{340}}\right)$ occurs in the eastern nucleus, which is similar to the Galactic value (100; Hildebrand 1983), while higher values of (371 \pm 118) $\left(\frac{\alpha_{\mathrm{CO}}}{0.8}\right)\left(\frac{0.9}{\kappa_{340}}\right)$ and $(339 \pm 60)\left(\frac{\alpha_{\mathrm{CO}}}{0.8}\right)\left(\frac{0.9}{\kappa_{340}}\right)$ occur in the western nucleus and the overlap region, respectively. The clear differences between the two nuclei may suggest a local gradient in the metallicity. For the overlap region, cold dust associated with diffuse gas clouds cannot avoid the collision. This tends to increase the $M_{\mathrm{H}_{2}} / M_{\text {dust }}$, because shocks destruct dust particles preferentially (Zhu et al. 2007). On the other hand, the low $M_{\mathrm{H}_{2}} / M_{\mathrm{dust}}$ in the eastern nucleus may be due to intense starbursts producing dust-rich environments.

\subsection{Fractional Abundances of $\mathrm{CS}, \mathrm{CH}_{3} \mathrm{OH}$, and $\mathrm{CN}$}

Table 15 shows the properties of the detected molecular lines which are not used in the RADEX calculations. 


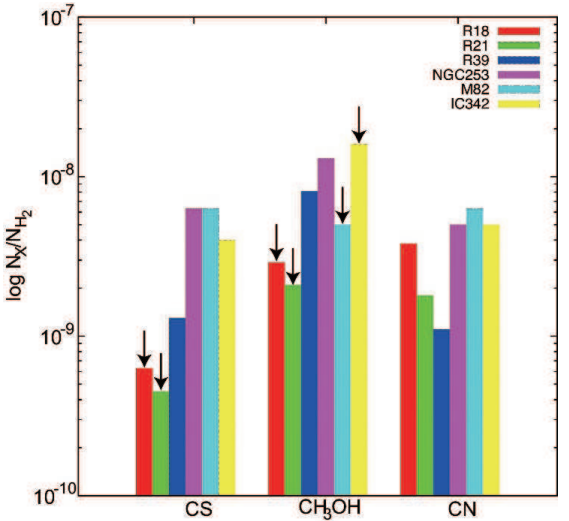

FIG. 13. - Logarithmic fractional abundances relative to $\mathrm{H}_{2}$ $\left([X] /\left[\mathrm{H}_{2}\right]\right)$ of selected extragalactic sources compared to those of specific regions of VV 114, as presented in Table 15. The red, green, and blue columns show R18, R21a, and R39a, respectively. The pink, light blue, and yellow columns show NGC 253, M 82, and IC 342, respectively. Arrows represent upper limits.

Either the dense gas component of VV 114 has extreme variations in excitation among the molecular clumps in the filament (see $\$ 6.1$ ), or there is widespread chemical differentiation across the filament. The fractional abundances $\left[N_{\mathrm{X}} / N_{\mathrm{H}_{2}}\right]$ of the different astrochemical species provide evidence of varying chemical influences due to star formation, physical conditions, and dynamics across the galaxy disks. We use the $\mathrm{H}_{2}$ column densities, derived from the RADEX calculations, which are $10^{20.8}$, $10^{21.1}$, and $10^{21.1} \mathrm{~cm}^{-2}$ at R18 (AGN), R21a (starburst), and R39a (overlap region), respectively. Column densities of each molecules are determined by equation (7) assuming an optically thin emission under LTE. The $T_{\text {ex }}$ values determined from equation $(3)$ are $38.7 \pm 1.9 \mathrm{~K}$, $58.8 \pm 2.9 \mathrm{~K}$, and $52.6 \pm 2.6 \mathrm{~K}$ at R18, R21a, and $\mathrm{R} 39 \mathrm{a}$, respectively. The derived $\left[N_{\mathrm{X}} / N_{\mathrm{H}_{2}}\right]$ are listed in Table 15.

In Figure 13, we show the fractional abundances for $\mathrm{CS}, \mathrm{CH}_{3} \mathrm{OH}$, and $\mathrm{CN}$ in $\mathrm{VV} 114$, and the same ratios for a sample of nearly galaxies, NGC 253, M82, and IC 342, taken from line surveys available in the literature (Henkel et al. 1988; Mauersberger et al. 1989; Huettemeister et al. 1997; Martín et al. 2006). M82 has a relatively old starburst at its core, with an average stellar population age of $\simeq 10$ Myr (Konstantopoulos et al. 2009). This creates strong UV fields, therefore the PDR dominates its chemistry (Aladro et al. 2011). Figure 13 shows that R39a has higher $\mathrm{CH}_{3} \mathrm{OH}$ abundance than M82, and small CS and CN abundances. A pure PDR similar to M82 may explain the molecular abundances we observe in R18.

The molecular abundances for the overlap region and NGC 253 share similar characteristics. NGC 253 is thought to be in an early stage of starburst evolution, and has young stellar populations in its nucleus $(\simeq 6 \mathrm{Myr}$; Fernández-Ontiveros et al. 2009). The chemistry in the nucleus of NGC 253 is dominated by large-scale shocks (Aladro et al. 2011), and we suggest that the overlap region of VV 114 is also dominated by shocks. The low $R_{\mathrm{HCN} / \mathrm{HCO}^{+}}$at the overlap region are further evidences for a shock dominated region (Krips et al. 2008).

\subsection{Merger-driven Tidal Dwarf Galaxy Formation}

Tidal dwarf galaxies (TDGs) are gas-rich irregular galaxies made out of stellar and gaseous material pulled out by tidal forces from the disks of the colliding parent galaxies into the intergalactic medium. They are found at the ends of long tails and host active star-forming regions (Braine et al. 2000). Hibbard et al. (2001) and Gao et al. (2001) found the HI gas mass of $4.1 \times 10^{8} \mathrm{M}_{\odot}$ and the molecular gas mass of $4 \times 10^{6} \mathrm{M}_{\odot}$ at the edge of the southern tail of NGC 4038/9.

We found an elevated $R_{3-2 / 1-0}(0.36 \pm 0.01)$, SFR $\left(0.10 \pm 0.05 \mathrm{M}_{\odot} \mathrm{yr}^{-1}\right)$, and $M_{\mathrm{H}_{2}}\left(\sim 3.8 \times 10^{7}\left(\frac{\alpha_{\mathrm{CO}}}{0.8}\right)\right.$ $\left.\mathrm{M}_{\odot}\right)$ at the edge of the southern tidal arm (R38). The derived SFR and $M_{\mathrm{H}_{2}}$ of R38 are comparable to those of TDG candidates in other galaxies (Braine et al. 2001). The gas depletion time of $(0.40 \pm 0.22)\left(\frac{\alpha_{\mathrm{CO}}}{0.8}\right)$ Gyr is shorter than the rest of the gas in the tidal arm ( $>$ $0.5\left(\frac{\alpha_{\mathrm{CO}}}{0.8}\right)$ Gyr $)$. According to the RADEX modeling, while the ranges of $T_{\text {kin }}$ and $n_{\mathrm{H}_{2}}$ are not confined well, the best fitting values $\left(35 \mathrm{~K}, 10^{2.5} \mathrm{~cm}^{-3}\right.$ ) are slightly higher than those in the middle of the tidal arm, R36a and R37a $\left(25-30 \mathrm{~K}, 10^{2.0}-10^{2.2} \mathrm{~cm}^{-3}\right)$. We suggest that R38 is a forming tidal dwarf galaxy at the edge of the tidal arm of VV 114. Future high sensitivity optical and high resolution HI observations will allow us to constrain the star formation and the atomic gas properties of R38.

\section{CONCLUSION}

We investigate the physical conditions of the molecular gas in the mid-stage merger VV 114. We present high-resolution observations of molecular gas and dust continuum emission in this galaxy using ALMA band 3 and band 7 . This study includes the first detection of extranuclear $\mathrm{CH}_{3} \mathrm{OH}(2-1)$ emission in interacting galaxies. The results can be summarized as follows:

1. We find that the $\mathrm{CO}(1-0)$ and $\mathrm{CO}(3-2)$ lines show significantly extended structures (i.e., the northern and southern tidal arms), the central filament across the galaxy disks, and double-peaks in the overlap region, while the ${ }^{13} \mathrm{CO}(1-0)$ line is only detected at the central filament. The filament is also identified by the strong $\mathrm{CN}\left(1_{3 / 2}-0_{1 / 2}\right)$, $\mathrm{HCO}^{+}$(4-3), $110 \mathrm{GHz}$, and $340 \mathrm{GHz}$ continuum emission.

2. Higher $R_{3-2 / 1-0}(0.5-0.8)$ and $R_{12 / 13}(20-50)$ are detected at the central filament. These higher ratios indicate that the central filament has highly excited (but not thermalized) molecular ISM, and the eastern nucleus is nearly thermalized when it is observed with a $800 \mathrm{pc}$ beam.

3. The unresolved eastern nucleus has the highest $R_{\mathrm{HCN} / \mathrm{HCO}^{+}}(1.34 \pm 0.09)$, while the dense gas clumps near the eastern nucleus have significantly lower values $(\sim 0.5)$. The broad $\mathrm{HCN}(4-3)$ and $\mathrm{HCO}^{+}(4-3)\left(\sim 290 \mathrm{~km} \mathrm{~s}^{-1}\right)$ emission lines seen in the unresolved eastern nucleus suggests an obscured AGN (see also paper I).

4. Radiative transfer analysis of the CO (1-0), CO (3$2)$, and ${ }^{13} \mathrm{CO}(1-0)$ emission enables us to map 
physical parameters of the "diffuse" gas of an interacting LIRG with 800 pc scale for the first time. The analysis suggests that "diffuse" gas clouds in the filament have warmer/denser conditions than those in the galaxy disks. This is consistent with predictions from merger simulations. Our analysis also suggest that the $[\mathrm{CO}] /\left[{ }^{13} \mathrm{CO}\right]$ is enhanced in the central filament. The extremely high $[\mathrm{CO}] /\left[{ }^{13} \mathrm{CO}\right]$ values are more important than the moderately optically thick ${ }^{12} \mathrm{CO}(1-0)$ emission to explain the high $R_{12 / 13}$ in VV 114.

5. Radiative transfer analysis of the $\mathrm{HCN}(4-3)$, $\mathrm{HCO}^{+}(4-3)$, and ${ }^{12} \mathrm{CO}(3-2)$ allow us to compare the dense gas clouds around AGN, starburst activities, and the overlap region. These results show that dense gas clouds around AGN have $n_{\mathrm{H}_{2}}$ $=10^{5.0}-10^{5.4} \mathrm{~cm}^{-3}$ and $T_{\text {kin }}>100 \mathrm{~K}$ with $[\mathrm{HCN}] /\left[\mathrm{HCO}^{+}\right]>5$, while gas clumps around starburst activities show $n_{\mathrm{H}_{2}}=10^{5.6}-10^{5.9} \mathrm{~cm}^{-3}$ and $T_{\text {kin }}=40-100 \mathrm{~K}$ with $[\mathrm{HCN}] /\left[\mathrm{HCO}^{+}\right]<4$. In addition, the analysis shows that the overlap region has $n_{\mathrm{H}_{2}}=10^{5.0}-10^{5.6} \mathrm{~cm}^{-3}$ and $T_{\text {kin }}=5-90 \mathrm{~K}$ with $[\mathrm{HCN}] /\left[\mathrm{HCO}^{+}\right]=1-9$.

6 . The spatially resolved Kennicutt-Schmidt law in VV 114 clearly connects the "starburst" sequence with the "normal disk" sequence. Most of the data points near the "starburst" sequence are found in the nuclei and the overlap region, whereas the data points near the "normal disk" sequence are found in the tidal arms. We also find the $R_{3-2 / 1-0}$ and $R_{12 / 13}$ are well correlated with the $\Sigma_{\mathrm{SFR}}$.

7. The $M_{\mathrm{H}_{2}} / M_{\text {dust }}$ of $(128 \pm 16)\left(\frac{\alpha_{\mathrm{CO}}}{0.8}\right)\left(\frac{0.9}{\kappa_{340}}\right)$ in the eastern nucleus of VV 114 is comparable to the Galactic value, but it is a factor of two higher than that in the overlap region of (339 \pm 60) $\left(\frac{\alpha_{\mathrm{CO}}}{0.8}\right)\left(\frac{0.9}{\kappa_{340}}\right)$. Since the $340 \mathrm{GHz}$ emission is spatially correlated with dense gas tracers, the cold dust in VV 114 appears to be closely related to the dense molecular component in the filament. The lowest $M_{\mathrm{H}_{2}} / M_{\text {dust }}$ in the eastern nucleus may be due to the dusty starburst.

8. Comparing the $\mathrm{CS}, \mathrm{CN}$, and $\mathrm{CH}_{3} \mathrm{OH}$ emission with other galaxies, we suggest that the overlap region is dominated by large-scale shocks similar to the nucleus of NGC 253. From the abundance analysis and distribution of the line ratios, we postulate that the $\mathrm{HCN}$-rich $\mathrm{AGN}$, the $\mathrm{HCO}^{+}$-rich starbursts, and the $\mathrm{CH}_{3} \mathrm{OH}$-rich overlap region are important drivers of the molecular chemistry of VV 114.

9. We find a region with relatively high excitation $(\simeq$ $35 \mathrm{~K}, \simeq 10^{2.5} \mathrm{~cm}^{-3}$ ) and star formation ( $\mathrm{SFR}=$ $0.10 \pm 0.05 \mathrm{M}_{\odot} \mathrm{yr}^{-1}$ ) at the edge of the southern tail. This region has a shorter $\tau_{\text {gas }}$ of $(0.40 \pm$ 0.22) $\left(\frac{\alpha_{\mathrm{CO}}}{0.8}\right)$ Gyr than the rest of the southern tail $\left(>1.35\left(\frac{\alpha_{\mathrm{CO}}}{0.8}\right) \mathrm{Gyr}\right)$, and we suggest that it is a forming tidal dwarf galaxy.

The authors thanks the anonymous referee for comments that improved the contents of this paper. TS thanks for Yoichi Tamura, Takuma Izumi, and Akio Taniguchi's help on the RADEX calculation. We used a script developed by Y. Tamura for this calculation (http://www.ioa.s.utokyo.ac.jp/ ${ }^{\sim}$ tamura/Wiki/?Science\%2FUsingRADEX). TS and other authors thank ALMA staff for their kind support. TS, J. Ueda, and K. Tateuchi are financially supported by a Research Fellowship from the Japan Society for the Promotion of Science for Young Scientists. D. Iono was supported by the ALMA Japan Research Grant of NAOJ Chile Observaory, NAOJ-ALMA0011 and JSPS KAKENHI Grant Number 2580016. This paper makes use of the following ALMA data: ADS/JAO.ALMA\#2011.0.00467.S. ALMA is a partnership of ESO (representing its member states), NSF (USA) and NINS (Japan), together with NRC (Canada) and NCS and ASIAA (Taiwan), in cooperation with the Republic of Chile. The Joint ALMA Observatory is operated by ESO, AUI/NRAO, and NAOJ.

\section{REFERENCES}

Aalto, S., Booth, R. S., Black, J. H., \& Johansson, L. E. B. 1995, A\&A, 300, 369

Aalto, S., Radford, S. J. E., Scoville, N. Z., \& Sargent, A. I. 1997, ApJ, 475, L107

Aalto, S., Beswick, R., Jutte, E. 2010, A\&A, 522, A59

Aladro, R., Martín, S., Martín-Pintado, J., et al. 2011, A\&A, 535, A84

Alonso-Herrero, A., Rieke, G. H., Rieke, M. J., \& Scoville, N. Z 2002, AJ, 124, 166

Armus, L., Mazzarella, J. M., Evans, A. S., et al. 2009, PASP, 121,559

Blake, G. A., Sutton, E. C., Masson, C. R., \& Phillips, T. G. 1987, ApJ, 315, 621

Bolatto, A. D., Wolfire, M., \& Leroy, A. K. 2013, ARA\&A, 51, 207

Bournaud, F., Powell, L. C., Chapon, D., \& Teyssier, R. 2011, IAU Symposium, 271, 160

Braine, J., Lisenfeld, U., Due, P.-A., \& Leon, S. 2000, Nature, 403, 867

Braine, J., Duc, P.-A., Lisenfeld, U., et al. 2001, A\&A, 378, 51

Briggs, D. S., \& Cornwell, T. J. 1992, Astronomical Data Analysis Software and Systems I, 25, 170

Casoli, F., Dupraz, C., \& Combes, F. 1992, A\&A, 264, 55
Condon, J. J., Huang, Z.-P., Yin, Q. F., \& Thuan, T. X. 1991, ApJ, 378, 65

Contini, M., \& Contini, T. 2003, MNRAS, 342, 299

Daddi, E., Elbaz, D., Walter, F., et al. 2010, ApJ, 714, L118

Davis, T. A., Heiderman, A., Evans, N. J., \& Iono, D. 2013,

MNRAS, 436, 570

Downes, D., \& Solomon, P. M. 1998, ApJ, 507, 615

Draine, B. T., \& Lee, H. M. 1984, ApJ, 285, 89

Engel, H., Davies, R. I., Genzel, R., et al. 2010, A\&A, 524, A56

Evans, A. S. 2008, Frontiers of Astrophysics: A Celebration of NRAO's 50th Anniversary, 395, 113

Fernández-Ontiveros, J. A., Prieto, M. A., \& Acosta-Pulido, J. A. 2009, MNRAS, 392, L16

Frayer, D. T., Ivison, R. J., Smail, I., Yun, M. S., \& Armus, L. 1999, AJ, 118, 139

Fujii, K., Minamidani, T., Mizuno, N., et al. 2014, ApJ, 796, 123

Gao, Y., Lo, K. Y., Lee, S.-W., \& Lee, T.-H. 2001, ApJ, 548, 172

Gao, Y., \& Solomon, P. M. 2004, ApJ, 606, 271

Garcia-Burillo, S., Combes, F., Usero, A., et al. 2014, arXiv:1405.7706

Genzel, R., Tacconi, L. J., Gracia-Carpio, J., et al. 2010, MNRAS, 407, 2091

Grimes, J. P., Heckman, T., Hoopes, C., et al. 2006, ApJ, 648, 310 
Harada, N., Thompson, T. A., \& Herbst, E. 2013, ApJ, 765, 108 Henkel, C., Schilke, P., \& Mauersberger, R. 1988, A\&A, 201, L23

Henkel, C., Asiri, H., Ao, Y., et al. 2014, A\&A, 565, A3

Henning, T., Michel, B., \& Stognienko, R. 1995,

Planet. Space Sci., 43, 1333

Hibbard, J. E., van der Hulst, J. M., Barnes, J. E., \& Rich, R. M. 2001, AJ, 122, 2969

Hildebrand, R. H. 1983, QJRAS, 24, 267

Högbom, J. A. 1974, A\&AS, 15, 417

Hopkins, P. F., Hernquist, L., Cox, T. J., et al. 2006, ApJS, 163, 1

Hopkins, P. F., Cox, T. J., Hernquist, L., et al. 2013, MNRAS, 430, 1901

Huettemeister, S., Mauersberger, R., \& Henkel, C. 1997, A\&A, 326,59

Imanishi, M., Nakanishi, K., Tamura, Y., Oi, N., \& Kohno, K. 2007, AJ, 134, 2366

Imanishi, M., \& Nakanishi, K. 2013, AJ, 146, 91

Imanishi, M., \& Nakanishi, K. 2014, AJ, 148, 9

Iono, D., Ho, P. T. P., Yun, M. S., et al. 2004, ApJ, 616, L63

Iono, D., Yun, M. S., \& Ho, P. T. P. 2005, ApJS, 158, 1

Iono, D., Wilson, C. D., Takakuwa, S., et al. 2007, ApJ, 659, 283

Iono, D., Saito, T., Yun, M. S., et al. 2013, PASJ, 65, L7

Izumi, T., Kohno, K., Martín, S., et al. 2013, PASJ, 65, 100

Jackson, J. M., Paglione, T. A. D., Carlstrom, J. E., \& Rieu, N.-Q. 1995, ApJ, 438, 695

Kartaltepe, J. S., Sanders, D. B., Le Floc'h, E., et al. 2010, ApJ, 721,98

Kennicutt, R. C., Jr. 1998, ApJ, 498, 541

Kohno, K., Matsushita, S., Vila-Vilaró, B., et al. 2001, The Central Kiloparsec of Starbursts and AGN: The La Palma Connection, 249, 672

Komugi, S., Sofue, Y., Nakanishi, H., Onodera, S., \& Egusa, F. 2005, PASJ, 57, 733

Konstantopoulos, I. S., Bastian, N., Smith, L. J., et al. 2009 , ApJ, 701, 1015

Krips, M., Neri, R., García-Burillo, S., et al. 2008, ApJ, 677, 262

Langer, W. D., \& Graedel, T. E. 1989, ApJS, 69, 241

Le Floc'h, E., Charmandaris, V., Laurent, O., et al. 2002, A\&A, 391,417

Leroy, A. K., Walter, F., Sandstrom, K., et al. 2013, AJ, 146, 19

Maloney, P. R., Hollenbach, D. J., \& Tielens, A. G. G. M. 1996, ApJ, 466, 561

Mao, R. Q., Henkel, C., Schulz, A., et al. 2000, A\&A, 358, 433

Martín, S., Mauersberger, R., Martín-Pintado, J., Henkel, C., \& García-Burillo, S. 2006, ApJS, 164, 450

Mauersberger, R., Henkel, C., Wilson, T. L., \& Harju, J. 1989, A\&A, 226, L5

McMullin, J. P., Waters, B., Schiebel, D., Young, W., \& Golap, K. 2007, Astronomical Data Analysis Software and Systems XVI, 376, 127

Minamidani, T., Mizuno, N., Mizuno, Y., et al. 2008, ApJS, 175, 485

Scoville, N., Aussel, H., Sheth, K., et al. 2014, ApJ, 783, 84

Papadopoulos, P. P., van der Werf, P. P., Xilouris, E. M., et al. 2012, MNRAS, 426, 2601
Rich, J. A., Kewley, L. J., \& Dopita, M. A. 2011, ApJ, 734, 87

Saito, T., Iono, D., Yun, M., et al. 2013, Astronomical Society of the Pacific Conference Series, 476, 287

Sakamoto, K., Aalto, S., Combes, F., Evans, A., \& Peck, A. 2014 arXiv:1403.7117

Sanders, D. B., Scoville, N. Z., \& Soifer, B. T. 1991, ApJ, 370, 158 Schmidt, M. 1959, ApJ, 129, 243

Schöier, F. L., van der Tak, F. F. S., van Dishoeck, E. F., \& Black, J. H. 2005, A\&A, 432, 369

Seaquist, E. R., \& Frayer, D. T. 2000, ApJ, 540, 765

Seaquist, E., Yao, L., Dunne, L., \& Cameron, H. 2004, MNRAS, 349, 1428

Sheffer, Y., Federman, S. R., Lambert, D. L., \& Cardelli, J. A. 1992, ApJ, 397, 482

Sliwa, K., Wilson, C. D., Krips, M., et al. 2013, ApJ, 777, 126

Soifer, B. T., Sanders, D. B., Madore, B. F., et al. 1987, ApJ, 320,238

Solomon, P. M., \& Vanden Bout, P. A. 2005, ARA\&A, 43, 677

Sternberg, A., \& Dalgarno, A. 1995, ApJS, 99, 565

Tamura, Y., Saito, T., Tsuru, T. G., et al. 2014, ApJ, 781, L39

Tateuchi, K., Motohara, K., Konishi, M., et al. 2012, Publication of Korean Astronomical Society, 27, 297

Teyssier, R., Chapon, D., \& Bournaud, F. 2010, ApJ, 720, L149

Thompson, A. R., Moran, J. M., \& Swenson, G. W., Jr. 2001, "Interferometry and synthesis in radio astronomy by A. Richard Thompson, James M. Moran, and George W. Swenson, Jr. 2nd ed. New York : Wiley, c2001.xxiii, 692 p. : ill. ; $25 \mathrm{~cm}$. "A Wiley-Interscience publication." Includes bibliographical references and indexes. ISBN : 0471254924"

Tsai, M., Hwang, C.-Y., Matsushita, S., Baker, A. J., \& Espada, D. 2012, ApJ, 746, 129

Ueda, J., Iono, D., Petitpas, G., et al. 2012, ApJ, 745, 65

Ueda, J., Iono, D., Yun, M. S., et al. 2014, ApJS, 214, 1

van Dishoeck, E. F., \& Black, J. H. 1988, ApJ, 334, 771

van der Tak, F. F. S., Black, J. H., Schöier, F. L., Jansen, D. J., \& van Dishoeck, E. F. 2007, A\&A, 468, 627

Warren, B. E., Wilson, C. D., Israel, F. P., et al. 2010, ApJ, 714, 571

Wild, W., Harris, A. I., Eckart, A., et al. 1992, A\&A, 265, 447

Wilson, C. D., Scoville, N., Madden, S. C., \& Charmandaris, V. 2003, ApJ, 599, 1049

Wilson, C. D., Petitpas, G. R., Iono, D., et al. 2008, ApJS, 178, 189

Wilson, C. D., Rangwala, N., Glenn, J., et al. 2014, ApJ, 789, L36

Wilson, T. L., Rohlfs, K., Huttemeister, S. 2009, Tools of Radio Astronomy, by Thomas L. Wilson; Kristen Rohlfs and Susanne Hüttemeister. ISBN 978-3-540-85121-9. Published by Springer-Verlag, Berlin, Germany, 2009.,

Wilson, T. L., \& Rood, R. 1994, ARA\&A, 32, 191

Yao, L., Seaquist, E. R., Kuno, N., \& Dunne, L. 2003, ApJ, 588, 771

Yun, M. S., Scoville, N. Z., \& Knop, R. A. 1994, ApJ, 430, L109

Zhu, M., Seaquist, E. R., \& Kuno, N. 2003, ApJ, 588, 243

Zhu, M., Gao, Y., Seaquist, E. R., \& Dunne, L. 2007, AJ, 134, 118 
TABLE 1

Log of ALMA OBSERVATions

\begin{tabular}{|c|c|c|c|c|c|c|c|c|c|}
\hline \multirow{2}{*}{$\begin{array}{l}\text { UT date } \\
\text { (1) }\end{array}$} & \multicolumn{2}{|c|}{ Spectral windows } & \multicolumn{3}{|c|}{ Configuration } & \multirow{2}{*}{$\begin{array}{r}T_{\text {sys }} \\
\\
{[\mathrm{K}]} \\
(7) \\
\end{array}$} & \multirow{2}{*}{$\begin{array}{r}\text { MRS } \\
\text { [arcsec.] } \\
(8)\end{array}$} & \multirow{2}{*}{$\begin{array}{r}\text { Amplitude caibrator } \\
(9)\end{array}$} & \multirow{2}{*}{$\begin{array}{c}T_{\text {obs }} \\
{[\underset{\min .]}{(10)}}\end{array}$} \\
\hline & $\begin{array}{r}\mathrm{LSB} \\
{[\mathrm{GHz}]} \\
(2)\end{array}$ & $\begin{array}{r}\text { USB } \\
{[\mathrm{GHz}]} \\
(3)\end{array}$ & $\begin{array}{r}N_{\text {ant }} \\
(4)\end{array}$ & $\begin{array}{r}\text { Array } \\
(5)\end{array}$ & $\begin{array}{r}L_{\text {baseline }} \\
{[\mathrm{m}]} \\
(6)\end{array}$ & & & & \\
\hline 2011 Nov 6 & $101.5,103.5$ & $114.0,115.1$ & 16 & CMP & $18-196$ & $65-89$ & 18 & Uranus & 41 \\
\hline 2012 May 4 & $101.5,103.5$ & $114.0,115.1$ & 15 & EXT & $39-402$ & $48-62$ & 8 & Neptune & 40 \\
\hline 2012 Mar 27 & $97.5, \quad 99.5$ & $110.2,111.5$ & 17 & EXT & $18-401$ & $54-73$ & 19 & Neptune & 22 \\
\hline 2012 Jul 2 & $97.5, \quad 99.5$ & $110.2,111.5$ & 20 & EXT & $16-402$ & $71-117$ & 21 & Neptune & 39 \\
\hline 2012 Nov 5 & $331.1,333.0$ & $343.5,345.3$ & 14 & CMP & $12-135$ & $125-172$ & 9 & Uranus & 66 \\
\hline 2012 Nov 5 & $331.1,333.0$ & $343.5,345.3$ & 14 & CMP & $12-135$ & $108-155$ & 9 & Uranus & 67 \\
\hline 2012 Nov 5 & $331.1,333.0$ & $343.5,345.3$ & 14 & CMP & $12-135$ & $124-175$ & 9 & Callisto & 67 \\
\hline 2012 Jun 1 & $342.0,344.0$ & $354.5,356.0$ & 18 & EXT & $15-402$ & $150-213$ & 7 & Uranus & 78 \\
\hline 2012 Jun 2 & $342.0,344.0$ & $354.5,356.0$ & 20 & EXT & $15-402$ & $108-160$ & 7 & Uranus & 80 \\
\hline 2012 Jun 3 & $342.0,344.0$ & $354.5,356.0$ & 20 & EXT & $15-402$ & $103-130$ & 7 & Uranus & 45 \\
\hline
\end{tabular}

Note. - Column 2 and 3: Central frequencies of the spectral windows (spw). All spw have the frequency coverage of $1.875 \mathrm{GHz}$. Column 4: Number of available antennas. Column 5: ALMA antenna configuration. CMP is the compact configuration and EXT is the extended configuration. Column 6: Range of projected length of baselines for VV 114. Column 7: DSB system temperature toward VV 114. Column 8: Maximum recoverable scale (MRS) of the configuration. This is defined by $\sim 0.6 \lambda /\left(\right.$ minimum $\left.L_{\text {baseline }}\right)$. Column 9: Observed calibrators for amplitude correction. Column 10: Total integration time on the galaxy.

TABLE 2

ALMA Observational Properties

\begin{tabular}{|c|c|c|c|c|c|c|c|}
\hline $\begin{array}{l}\text { Emission } \\
\text { (1) }\end{array}$ & $\begin{array}{r}\text { Band } \\
(2)\end{array}$ & $\begin{array}{r}\nu_{\text {rest }} \\
{[\mathrm{GHz}]} \\
(3)\end{array}$ & $\begin{array}{r}\text { Beam size } \\
\text { [arcsecond] } \\
(4)\end{array}$ & $\begin{array}{r}\text { P.A. } \\
\text { [deg] } \\
(5)\end{array}$ & $\begin{array}{r}\Delta V \\
{\left[\mathrm{~km} \mathrm{~s}^{-1}\right]} \\
(6)\end{array}$ & $\begin{array}{r}\text { Noise rms } \\
{\left[\text { mJy beam }^{-1}\right]} \\
(7)\end{array}$ & $\frac{[\mathrm{mK}]}{(8)}$ \\
\hline $\mathrm{CH}_{3} \mathrm{OH}\left(2_{k}-1_{k}\right)$ & 3 & 96.74 & $2.03 \times 1.34$ & 85.7 & 30 & 1.0 & 46 \\
\hline $\mathrm{CS}(2-1)$ & 3 & 97.98 & $2.01 \times 1.37$ & 83.6 & 30 & 0.9 & 40 \\
\hline${ }^{13} \mathrm{CO}(1-0)$ & 3 & 110.20 & $1.77 \times 1.20$ & 85.8 & 30 & 1.0 & 46 \\
\hline $\mathrm{CN}\left(1_{1 / 2}-0_{1 / 2}\right)$ & 3 & 113.14 & $1.97 \times 1.27$ & -85.8 & 30 & 1.0 & 37 \\
\hline $\mathrm{CN}\left(1_{3 / 2}-0_{1 / 2}\right)$ & 3 & 113.49 & $1.98 \times 1.29$ & -84.7 & 30 & 1.1 & 39 \\
\hline $\mathrm{CO}(1-0)$ & 3 & 115.27 & $1.97 \times 1.35$ & 82.3 & 10 & 2.3 & 76 \\
\hline $\mathrm{CS}(7-6)$ & 7 & 342.88 & $0.47 \times 0.39$ & 54.2 & 30 & 0.7 & 38 \\
\hline $\mathrm{CO}(3-2)$ & 7 & 345.80 & $1.64 \times 1.17$ & 112.6 & 10 & 2.1 & 11 \\
\hline $\mathrm{HCN}(4-3)$ & 7 & 354.51 & $0.46 \times 0.38$ & 51.5 & 30 & 0.8 & 42 \\
\hline $\mathrm{HCO}^{+}(4-3)$ & 7 & 356.73 & $0.45 \times 0.37$ & 53.4 & 30 & 0.9 & 50 \\
\hline Continuum & 3 & 110 & $1.89 \times 1.28$ & 81.8 & .. & 0.05 & 2.1 \\
\hline Continuum & 7 & 340 & $1.33 \times 1.12$ & 119.6 & ... & 0.11 & 0.8 \\
\hline Continuum & 7 & 340 & $0.45 \times 0.38$ & 56.2 & .. & 0.07 & 4.3 \\
\hline
\end{tabular}

Note. - Column 1: Identified emission. Column 2: Band which includes the molecular line and continuum emission. Column 3: Rest frequency of the line or mean frequency of the continuum. Column 4: Major and minor axes (FWHM) of the synthesized beam. Column 5: Position angle of the synthesized beam. Column 6: Velocity resolution of our binning images. Column 7 and 8: Noise rms intensity in the data which have velocity resolutions shown in Column 6 . The noise in Column 8 is in Rayleigh-Jeans brightness temperature. 
TABLE 3

Radio Continuum Flux of VV 114

\begin{tabular}{|c|c|c|c|}
\hline $\begin{array}{l}\text { ID } \\
(1)\end{array}$ & $\begin{array}{r}S_{8.44} \\
{[\mathrm{mJy}]} \\
(2)\end{array}$ & $\begin{array}{r}S_{110} \\
{[\mathrm{mJy}]} \\
(3)\end{array}$ & $\begin{array}{r}S_{340} \\
{[\mathrm{mJy}]} \\
(4)\end{array}$ \\
\hline $\mathrm{R} 7$ & $0.62 \pm 0.08$ & $<0.18$ & $0.58 \pm 0.14$ \\
\hline $\mathrm{R} 8$ & $0.70 \pm 0.08$ & $<0.18$ & $<0.42$ \\
\hline R9 & $0.82 \pm 0.08$ & $0.21 \pm 0.06$ & $<0.42$ \\
\hline R10 & $0.81 \pm 0.08$ & $0.20 \pm 0.06$ & $<0.42$ \\
\hline R11 & $0.92 \pm 0.08$ & $<0.18$ & $<0.42$ \\
\hline $\mathrm{R} 17$ & $0.42 \pm 0.08$ & $<0.18$ & $<0.42$ \\
\hline $\mathrm{R} 18^{\dagger}$ & $3.37 \pm 0.08$ & $1.48 \pm 0.06$ & $5.17 \pm 0.14$ \\
\hline R19 & $2.40 \pm 0.08$ & $1.03 \pm 0.06$ & $3.03 \pm 0.14$ \\
\hline $\mathrm{R} 20$ & $1.14 \pm 0.08$ & $0.54 \pm 0.06$ & $2.41 \pm 0.14$ \\
\hline $\mathrm{R} 21^{\dagger \dagger}$ & $5.00 \pm 0.08$ & $1.86 \pm 0.06$ & $8.20 \pm 0.14$ \\
\hline $\mathrm{R} 22$ & $1.85 \pm 0.08$ & $0.81 \pm 0.06$ & $4.37 \pm 0.14$ \\
\hline $\mathrm{R} 23$ & $1.34 \pm 0.08$ & $0.50 \pm 0.06$ & $3.55 \pm 0.14$ \\
\hline $\mathrm{R} 24$ & $0.67 \pm 0.08$ & $<0.18$ & $1.19 \pm 0.14$ \\
\hline $\mathrm{R} 25$ & $1.07 \pm 0.08$ & $0.39 \pm 0.06$ & $3.01 \pm 0.14$ \\
\hline $\mathrm{R} 26$ & $0.81 \pm 0.08$ & $0.33 \pm 0.06$ & $1.73 \pm 0.14$ \\
\hline $\mathrm{R} 27$ & $1.29 \pm 0.08$ & $0.41 \pm 0.06$ & $1.40 \pm 0.14$ \\
\hline $\mathrm{R} 28$ & $0.81 \pm 0.08$ & $0.21 \pm 0.06$ & $0.76 \pm 0.14$ \\
\hline $\mathrm{R} 29$ & $0.35 \pm 0.08$ & $<0.18$ & $0.71 \pm 0.14$ \\
\hline R30 & $<0.24$ & $<0.18$ & $0.70 \pm 0.14$ \\
\hline $\mathrm{R} 39^{\dagger \dagger \dagger}$ & $1.18 \pm 0.08$ & $0.21 \pm 0.06$ & $3.35 \pm 0.14$ \\
\hline
\end{tabular}

Note. - Column 2: $8.44 \mathrm{GHz}$ continuum flux (Condon et al. 1991). Column 3: $110 \mathrm{GHz}$ continuum flux obtained by ALMA/band 3. Column 4: $340 \mathrm{GHz}$ continuum flux obtained by ALMA/band 7.; We only show the statistical error in this table. The systematic error of absolute flux calibration is estimated to be $\sim 5 \%$ in band 3 and $\sim 10 \%$ in band 7 . †represents boxes contained the obscured AGN defined by paper I. $\dagger \dagger$ represents boxes contained the nuclear starbursts defined by paper I. †††represents boxes at the overlap region. 
TABLE 4

$\mathrm{CO}(1-0),{ }^{13} \mathrm{CO}(1-0)$, And ${ }^{12} \mathrm{CO}(3-2)$ Integrated Intensities

\begin{tabular}{|c|c|c|c|c|c|}
\hline ID & $\begin{array}{r}{ }^{12} \mathrm{CO}(1-0) \\
{\left[\begin{array}{ll}\mathrm{Jy} \\
\mathrm{km} \mathrm{s}\end{array}\right.} \\
(2)\end{array}$ & $\begin{array}{r}{ }^{12} \mathrm{CO}(3-2) \\
{\left[\begin{array}{ll}\mathrm{Jy} \\
\mathrm{km} \mathrm{s}\end{array}\right.} \\
(3)\end{array}$ & $\begin{array}{r}{ }^{13} \mathrm{CO}(1-0) \\
{\left[\begin{array}{ll}\mathrm{Jy} \mathrm{km} \mathrm{s} & -1 \\
(4)\end{array}\right.}\end{array}$ & $\begin{array}{r}R_{3-2 / 1-0} \\
(5)\end{array}$ & $\begin{array}{r}R_{12 / 13} \\
\quad(6)\end{array}$ \\
\hline R1 & $2.12 \pm 0.06$ & $5.92 \pm 0.08$ & . & $0.31 \pm 0.01$ & $\ldots$ \\
\hline $\mathrm{R} 2$ & $1.41 \pm 0.05$ & $7.47 \pm 0.06$ & $0.29 \pm 0.02$ & $0.59 \pm 0.02$ & $4 \pm 1$ \\
\hline R3 & $3.62 \pm 0.09$ & $5.45 \pm 0.09$ & & $0.17 \pm 0.01$ & \\
\hline R4 & $3.54 \pm 0.08$ & $8.69 \pm 0.10$ & $0.25 \pm 0.04$ & $0.27 \pm 0.01$ & $13 \pm 2$ \\
\hline R5 & $3.46 \pm 0.08$ & $9.16 \pm 0.09$ & $0.40 \pm 0.03$ & $0.29 \pm 0.01$ & $7 \pm 1$ \\
\hline R6 & $3.66 \pm 0.10$ & $10.71 \pm 0.11$ & & $0.33 \pm 0.01$ & \\
\hline R7 & $5.26 \pm 0.10$ & $19.64 \pm 0.12$ & $0.40 \pm 0.03$ & $0.41 \pm 0.01$ & $12 \pm 1$ \\
\hline $\mathrm{R} 8$ & $3.71 \pm 0.09$ & $13.44 \pm 0.12$ & $0.38 \pm 0.03$ & $0.40 \pm 0.01$ & $8 \pm 1$ \\
\hline R9 & $9.59 \pm 0.11$ & $33.88 \pm 0.12$ & $0.43 \pm 0.04$ & $0.39 \pm 0.01$ & $20 \pm 2$ \\
\hline R10 & $12.69 \pm 0.11$ & $47.74 \pm 0.12$ & $0.37 \pm 0.05$ & $0.42 \pm 0.01$ & $32 \pm 4$ \\
\hline R11 & $16.26 \pm 0.13$ & $58.93 \pm 0.14$ & $0.49 \pm 0.04$ & $0.40 \pm 0.01$ & $30 \pm 3$ \\
\hline R12 & $4.61 \pm 0.10$ & $13.53 \pm 0.10$ & & $0.33 \pm 0.01$ & \\
\hline R13 & $5.22 \pm 0.10$ & $16.02 \pm 0.11$ & $0.38 \pm 0.05$ & $0.34 \pm 0.01$ & $12 \pm 2$ \\
\hline R14 & $4.70 \pm 0.09$ & $12.22 \pm 0.09$ & $0.35 \pm 0.03$ & $0.29 \pm 0.01$ & $12 \pm 1$ \\
\hline $\mathrm{R} 15$ & $5.03 \pm 0.09$ & $10.62 \pm 0.09$ & $0.35 \pm 0.03$ & $0.23 \pm 0.01$ & $13 \pm 1$ \\
\hline R16 & $5.59 \pm 0.09$ & $11.23 \pm 0.08$ & $0.38 \pm 0.02$ & $0.22 \pm 0.01$ & $14 \pm 1$ \\
\hline $\mathrm{R} 17$ & $6.44 \pm 0.10$ & $14.33 \pm 0.11$ & $0.38 \pm 0.05$ & $0.25 \pm 0.01$ & $15 \pm 2$ \\
\hline $\mathrm{R} 18^{\dagger}$ & $16.54 \pm 0.13$ & $106.39 \pm 0.15$ & $0.60 \pm 0.05$ & $0.71 \pm 0.01$ & $25 \pm 2$ \\
\hline R19 & $11.09 \pm 0.12$ & $54.98 \pm 0.14$ & $0.60 \pm 0.05$ & $0.55 \pm 0.01$ & $17 \pm 2$ \\
\hline $\mathrm{R} 20$ & $18.33 \pm 0.13$ & $93.14 \pm 0.14$ & $0.64 \pm 0.06$ & $0.56 \pm 0.01$ & $26 \pm 2$ \\
\hline $\mathrm{R} 21^{\dagger \dagger}$ & $33.04 \pm 0.15$ & $225.47 \pm 0.16$ & $1.09 \pm 0.07$ & $0.76 \pm 0.01$ & $28 \pm 2$ \\
\hline $\mathrm{R} 22$ & $28.67 \pm 0.15$ & $162.41 \pm 0.16$ & $0.90 \pm 0.07$ & $0.63 \pm 0.01$ & $29 \pm 2$ \\
\hline R23 & $33.65 \pm 0.16$ & $177.02 \pm 0.17$ & $0.95 \pm 0.07$ & $0.58 \pm 0.01$ & $32 \pm 2$ \\
\hline R24 & $16.12 \pm 0.14$ & $68.28 \pm 0.15$ & $0.48 \pm 0.07$ & $0.47 \pm 0.01$ & $31 \pm 4$ \\
\hline $\mathrm{R} 25$ & $25.23 \pm 0.16$ & $138.20 \pm 0.16$ & $0.86 \pm 0.06$ & $0.61 \pm 0.01$ & $27 \pm 2$ \\
\hline $\mathrm{R} 26$ & $20.44 \pm 0.14$ & $97.56 \pm 0.15$ & $0.69 \pm 0.06$ & $0.53 \pm 0.01$ & $27 \pm 3$ \\
\hline $\mathrm{R} 27$ & $12.45 \pm 0.12$ & $67.10 \pm 0.13$ & $0.54 \pm 0.06$ & $0.60 \pm 0.01$ & $21 \pm 2$ \\
\hline $\mathrm{R} 28$ & $5.78 \pm 0.10$ & $36.14 \pm 0.14$ & $0.35 \pm 0.03$ & $0.69 \pm 0.01$ & $15 \pm 1$ \\
\hline R29 & & & & & \\
\hline R30 & $6.39 \pm 0.12$ & $30.50 \pm 0.12$ & $0.28 \pm 0.03$ & $0.53 \pm 0.01$ & $21 \pm 3$ \\
\hline R31 & $4.93 \pm 0.11$ & $19.03 \pm 0.13$ & $0.30 \pm 0.04$ & $0.43 \pm 0.01$ & $15 \pm 2$ \\
\hline R32 & $2.34 \pm 0.05$ & $7.34 \pm 0.08$ & $0.21 \pm 0.02$ & $0.35 \pm 0.01$ & $10 \pm 1$ \\
\hline R33 & $1.44 \pm 0.05$ & $2.03 \pm 0.05$ & & $0.16 \pm 0.01$ & \\
\hline R34 & $2.81 \pm 0.08$ & $11.65 \pm 0.10$ & $0.32 \pm 0.03$ & $0.46 \pm 0.01$ & $8 \pm 1$ \\
\hline R35 & $5.36 \pm 0.09$ & $9.40 \pm 0.09$ & $0.30 \pm 0.03$ & $0.19 \pm 0.01$ & $16 \pm 2$ \\
\hline R36 & $5.55 \pm 0.12$ & $17.95 \pm 0.14$ & $0.31 \pm 0.02$ & $0.36 \pm 0.01$ & $17 \pm 1$ \\
\hline R37 & $4.84 \pm 0.11$ & $8.65 \pm 0.11$ & $0.31 \pm 0.03$ & $0.20 \pm 0.01$ & $14 \pm 2$ \\
\hline R38 & $2.66 \pm 0.07$ & $8.51 \pm 0.09$ & $0.33 \pm 0.02$ & $0.36 \pm 0.01$ & $7 \pm 1$ \\
\hline $\mathrm{R} 39^{\dagger \dagger \dagger}$ & $27.54 \pm 0.15$ & $155.31 \pm 0.15$ & $0.97 \pm 0.06$ & $0.63 \pm 0.01$ & $26 \pm 2$ \\
\hline
\end{tabular}

Note. - Column 1: These numbers are labeled at the ratio map of Figure 7. Column 2: Integrated ${ }^{12} \mathrm{CO}(1-0)$ intensity at an emission region. Column 3: Integrated ${ }^{12} \mathrm{CO}(3-2)$ intensity at an emission region. Column 4: Integrated ${ }^{13} \mathrm{CO}(3-2)$ intensity at an emission region. Column 5: The ${ }^{12} \mathrm{CO}(3-2) / \mathrm{CO}(1-0)$ integrated intensity ratio. Column 6: The ${ }^{12} \mathrm{CO}(1-0) /{ }^{13} \mathrm{CO}(1-0)$ integrated intensity ratio.; We only show the statistical error in this table. The systematic error of absolute flux calibration is estimated to be $\sim 5 \%$ in band 3 and $\sim 10 \%$ in band 7 . †represents boxes contained the obscured AGN defined by paper I. ††represents boxes contained the nuclear starbursts defined by paper I. $\dagger \dagger \dagger$ represents boxes at the overlap region. 
TABLE 5

HCN (4-3) And $\mathrm{HCO}^{+}(4-3)$ Integrated INTENSITIES

\begin{tabular}{|c|c|c|c|}
\hline $\begin{array}{l}\text { ID } \\
\text { (1) }\end{array}$ & $\begin{array}{r}\mathrm{HCN}(4-3) \\
{\left[\begin{array}{l}\mathrm{Jy} \mathrm{km} \mathrm{s} \\
-1\end{array}\right]} \\
(2)\end{array}$ & $\begin{array}{r}\mathrm{HCO}^{+}(4-3) \\
{\left[\mathrm{Jy} \mathrm{km} \mathrm{s}^{-1}\right]} \\
(3)\end{array}$ & $\begin{array}{r}R_{\mathrm{HCN} / \mathrm{HCO}}+ \\
(4)\end{array}$ \\
\hline $\begin{array}{l}\mathrm{S}^{\dagger} \\
\mathrm{S} 1 \\
\mathrm{~S} 2 \\
\mathrm{~S} 3^{\dagger \dagger} \\
\mathrm{S} 4 \\
\mathrm{~S} 5^{\dagger \dagger} \\
\mathrm{S} 6 \\
\mathrm{~S} 7 \\
\mathrm{~S} 8 \\
\mathrm{~S} 9 \\
\mathrm{~S} 10 \\
\mathrm{~S} 11^{\dagger \dagger \dagger} \\
\mathrm{S} 12^{\dagger \dagger \dagger} \\
\mathrm{S} 13 \\
\mathrm{~S} 14\end{array}$ & $\begin{aligned} 1.34 & \pm 0.09 \\
0.11 & \pm 0.03 \\
& <0.05 \\
0.49 & \pm 0.07 \\
0.13 & \pm 0.06 \\
0.81 & \pm 0.07 \\
0.40 & \pm 0.07 \\
0.19 & \pm 0.07 \\
0.06 & \pm 0.04 \\
0.07 & \pm 0.05 \\
& <0.04 \\
& <0.05 \\
& <0.04 \\
& <0.04 \\
& <0.05\end{aligned}$ & $\begin{array}{l}0.88 \pm 0.10 \\
0.34 \pm 0.06 \\
0.12 \pm 0.06 \\
1.05 \pm 0.08 \\
0.63 \pm 0.08 \\
2.36 \pm 0.09 \\
0.92 \pm 0.08 \\
0.42 \pm 0.08 \\
0.14 \pm 0.06 \\
0.13 \pm 0.06 \\
0.28 \pm 0.07 \\
0.15 \pm 0.06 \\
0.32 \pm 0.07 \\
0.17 \pm 0.06 \\
0.19 \pm 0.07\end{array}$ & $\begin{aligned} & 1.52 \pm 0.20 \\
& 0.34 \pm 0.10 \\
&<0.43 \\
& 0.46 \pm 0.07 \\
& 0.20 \pm 0.09 \\
& 0.34 \pm 0.03 \\
& 0.43 \pm 0.09 \\
& 0.45 \pm 0.18 \\
& 0.44 \pm 0.31 \\
& 0.54 \pm 0.46 \\
&<0.14 \\
&<0.33 \\
&<0.12 \\
&<0.24 \\
&<0.26\end{aligned}$ \\
\hline
\end{tabular}

Note. - Column 1: These numbers are labeled at the ratio map of Figure 7. Column 2: Integrated HCN (43 ) intensity at an emission region. Column 3: Integrated $\mathrm{HCO}^{+}(4-3)$ intensity at an emission region. Column 4: The $\mathrm{HCN}(4-3) / \mathrm{HCO}^{+}(4-3)$ integrated intensity ratio.; We only show the statistical error in this table. The systematic error of absolute flux calibration is estimated to be $\sim 5 \%$ in band 3 and $\sim 10 \%$ in band 7 . †represents boxes contained the obscured AGN defined by paper I. ††represents boxes contained the nuclear starbursts defined by paper I. †††represents boxes at the overlap region. 
TABLE 6

Peak Brightness Temperature of ${ }^{12} \mathrm{CO}(1-0),{ }^{13} \mathrm{CO}(1-0)$, and ${ }^{12} \mathrm{CO}(3-2)$ Emission

\begin{tabular}{|c|c|c|c|c|c|}
\hline (1) & $\begin{array}{r}\text { Peak } T_{\mathrm{b}, \mathrm{CO}}(1-0) \\
{[\mathrm{K}]} \\
(2)\end{array}$ & $\begin{array}{r}\text { Peak } T_{\mathrm{b}, \mathrm{CO}}(3-2) \\
{[\mathrm{K}]} \\
(3)\end{array}$ & $\begin{array}{r}\text { Peak } T_{\mathrm{b},{ }^{13} \mathrm{CO}(1-0)} \\
{[\mathrm{K}]} \\
(4)\end{array}$ & $\begin{array}{r}\text { Peak } R_{3-2 / 1-0} \\
(5)\end{array}$ & $\begin{array}{r}\text { Peak } R_{12 / 13} \\
(6)\end{array}$ \\
\hline $\mathrm{R} 1$ & $0.40 \pm 0.07$ & $0.13 \pm 0.01$ & $<0.09$ & $0.32 \pm 0.07$ & $>4$ \\
\hline $\mathrm{R} 2$ & $0.69 \pm 0.07$ & $0.25 \pm 0.01$ & $<0.09$ & $0.37 \pm 0.04$ & $>8$ \\
\hline R3 & $0.61 \pm 0.07$ & $0.07 \pm 0.01$ & $<0.09$ & $0.12 \pm 0.02$ & $>7$ \\
\hline $\mathrm{R} 4$ & $0.98 \pm 0.07$ & $0.30 \pm 0.01$ & $<0.09$ & $0.30 \pm 0.02$ & $>11$ \\
\hline R5 & $1.19 \pm 0.07$ & $0.42 \pm 0.01$ & $0.06 \pm 0.03$ & $0.35 \pm 0.02$ & $20 \pm 2$ \\
\hline R6 & $0.87 \pm 0.07$ & $0.21 \pm 0.01$ & $<0.09$ & $0.24 \pm 0.02$ & $>10$ \\
\hline R7a & $1.34 \pm 0.07$ & $0.32 \pm 0.01$ & $<0.09$ & $0.24 \pm 0.01$ & $>15$ \\
\hline R7b & $0.45 \pm 0.07$ & $0.36 \pm 0.01$ & $0.06 \pm 0.03$ & $0.79 \pm 0.12$ & $7 \pm 1$ \\
\hline R8a & $0.21 \pm 0.07$ & $<0.03$ & $<0.09$ & $>0.14$ & $>2$ \\
\hline $\mathrm{R} 8 \mathrm{~b}$ & $0.96 \pm 0.07$ & $0.24 \pm 0.01$ & $0.08 \pm 0.03$ & $0.25 \pm 0.02$ & $11 \pm 1$ \\
\hline R9a & $0.40 \pm 0.07$ & $0.12 \pm 0.01$ & $<0.09$ & $0.30 \pm 0.06$ & \\
\hline $\mathrm{R} 9 \mathrm{~b}$ & $2.60 \pm 0.07$ & $0.99 \pm 0.01$ & $0.08 \pm 0.03$ & $0.38 \pm 0.01$ & $33 \pm 2$ \\
\hline R10a & $0.80 \pm 0.07$ & $0.24 \pm 0.01$ & $0.05 \pm 0.03$ & $0.30 \pm 0.03$ & $17 \pm 2$ \\
\hline R10b & $2.81 \pm 0.07$ & $1.27 \pm 0.01$ & $0.09 \pm 0.03$ & $0.45 \pm 0.01$ & $30 \pm 1$ \\
\hline R11a & $2.11 \pm 0.07$ & $0.82 \pm 0.01$ & $0.12 \pm 0.03$ & $0.39 \pm 0.01$ & $18 \pm 1$ \\
\hline R11b & $3.34 \pm 0.07$ & $1.36 \pm 0.01$ & $0.04 \pm 0.03$ & $0.41 \pm 0.01$ & $89 \pm 7$ \\
\hline R12a & $0.55 \pm 0.07$ & $0.23 \pm 0.01$ & $<0.09$ & $0.41 \pm 0.06$ & $>6$ \\
\hline $\mathrm{R} 12 \mathrm{~b}$ & $1.30 \pm 0.07$ & $0.41 \pm 0.01$ & $<0.09$ & $0.32 \pm 0.02$ & $>14$ \\
\hline R13a & $1.12 \pm 0.07$ & $0.34 \pm 0.01$ & $0.06 \pm 0.03$ & $0.30 \pm 0.02$ & $18 \pm 1$ \\
\hline R13b & $0.67 \pm 0.07$ & $0.30 \pm 0.01$ & $<0.09$ & $0.45 \pm 0.05$ & $>7$ \\
\hline R14 & $1.61 \pm 0.07$ & $0.39 \pm 0.01$ & $0.08 \pm 0.03$ & $0.24 \pm 0.01$ & $20 \pm 1$ \\
\hline R15 & $1.77 \pm 0.07$ & $0.42 \pm 0.01$ & $0.08 \pm 0.03$ & $0.24 \pm 0.01$ & $22 \pm 1$ \\
\hline R16 & $1.38 \pm 0.07$ & $0.33 \pm 0.01$ & $0.12 \pm 0.03$ & $0.24 \pm 0.01$ & $12 \pm 1$ \\
\hline $\mathrm{R} 17$ & $1.64 \pm 0.07$ & $0.35 \pm 0.01$ & $0.05 \pm 0.03$ & $0.21 \pm 0.01$ & $36 \pm 3$ \\
\hline $\mathrm{R} 18^{\dagger}$ & $3.52 \pm 0.07$ & $1.79 \pm 0.01$ & $0.14 \pm 0.03$ & $0.51 \pm 0.01$ & $25 \pm 1$ \\
\hline R19a & $1.01 \pm 0.07$ & $0.54 \pm 0.01$ & $0.09 \pm 0.03$ & $0.54 \pm 0.04$ & $11 \pm 1$ \\
\hline R19b & $1.99 \pm 0.07$ & $0.98 \pm 0.01$ & $0.09 \pm 0.03$ & $0.49 \pm 0.02$ & $23 \pm 1$ \\
\hline $\mathrm{R} 20 \mathrm{a}$ & $3.33 \pm 0.07$ & $1.99 \pm 0.01$ & $0.11 \pm 0.03$ & $0.60 \pm 0.01$ & $30 \pm 1$ \\
\hline $\mathrm{R} 20 \mathrm{~b}$ & $1.13 \pm 0.07$ & $0.67 \pm 0.01$ & $<0.09$ & $0.59 \pm 0.04$ & $>13$ \\
\hline $\mathrm{R} 21 \mathrm{a}^{\dagger \dagger}$ & $5.53 \pm 0.07$ & $4.49 \pm 0.01$ & $0.23 \pm 0.03$ & $0.81 \pm 0.01$ & $24 \pm 1$ \\
\hline $\mathrm{R} 21 \mathrm{~b}^{\dagger \dagger}$ & $1.22 \pm 0.07$ & $0.95 \pm 0.01$ & $<0.09$ & $0.78 \pm 0.05$ & $>14$ \\
\hline $\mathrm{R} 21 \mathrm{c}^{\dagger \dagger}$ & $0.40 \pm 0.07$ & $0.72 \pm 0.01$ & $<0.09$ & $1.80 \pm 0.05$ & $>4$ \\
\hline $\mathrm{R} 22 \mathrm{a}$ & $3.60 \pm 0.07$ & $1.93 \pm 0.01$ & $0.12 \pm 0.03$ & $0.54 \pm 0.01$ & $29 \pm 1$ \\
\hline $\mathrm{R} 22 \mathrm{~b}$ & $3.83 \pm 0.07$ & $2.61 \pm 0.01$ & $0.16 \pm 0.03$ & $0.68 \pm 0.01$ & $24 \pm 1$ \\
\hline R23a & $4.17 \pm 0.07$ & $2.56 \pm 0.01$ & $0.15 \pm 0.03$ & $0.61 \pm 0.01$ & $28 \pm 1$ \\
\hline $\mathrm{R} 23 \mathrm{~b}$ & $3.39 \pm 0.07$ & $1.87 \pm 0.01$ & $0.12 \pm 0.03$ & $0.55 \pm 0.01$ & $28 \pm 1$ \\
\hline R24a & $1.23 \pm 0.07$ & $0.46 \pm 0.01$ & $0.05 \pm 0.03$ & $0.37 \pm 0.02$ & $23 \pm 2$ \\
\hline $\mathrm{R} 24 \mathrm{~b}$ & $1.60 \pm 0.07$ & $0.96 \pm 0.01$ & $0.06 \pm 0.03$ & $0.60 \pm 0.03$ & $28 \pm 2$ \\
\hline $\mathrm{R} 24 \mathrm{c}$ & $1.78 \pm 0.07$ & $0.58 \pm 0.01$ & $0.06 \pm 0.03$ & $0.32 \pm 0.01$ & $32 \pm 2$ \\
\hline $\mathrm{R} 25 \mathrm{a}$ & $4.89 \pm 0.07$ & $3.18 \pm 0.01$ & $0.23 \pm 0.03$ & $0.65 \pm 0.01$ & $21 \pm 1$ \\
\hline $\mathrm{R} 25 \mathrm{~b}$ & $1.77 \pm 0.07$ & $0.59 \pm 0.01$ & $0.06 \pm 0.03$ & $0.33 \pm 0.01$ & $31 \pm 2$ \\
\hline R26a & $4.49 \pm 0.07$ & $2.57 \pm 0.01$ & $0.14 \pm 0.03$ & $0.57 \pm 0.01$ & $33 \pm 1$ \\
\hline R26b & $1.07 \pm 0.07$ & $0.31 \pm 0.01$ & $0.07 \pm 0.03$ & $0.29 \pm 0.02$ & $15 \pm 1$ \\
\hline $\mathrm{R} 27 \mathrm{a}$ & $1.96 \pm 0.07$ & $1.31 \pm 0.01$ & $0.04 \pm 0.03$ & $0.67 \pm 0.02$ & $47 \pm 4$ \\
\hline $\mathrm{R} 27 \mathrm{~b}$ & $1.53 \pm 0.07$ & $0.75 \pm 0.01$ & $0.13 \pm 0.03$ & $0.49 \pm 0.02$ & $12 \pm 1$ \\
\hline $\mathrm{R} 28 \mathrm{a}$ & $0.76 \pm 0.07$ & $0.37 \pm 0.01$ & $0.05 \pm 0.03$ & $0.48 \pm 0.05$ & $16 \pm 2$ \\
\hline $\mathrm{R} 28 \mathrm{~b}$ & $0.86 \pm 0.07$ & $0.54 \pm 0.01$ & $<0.09$ & $0.62 \pm 0.05$ & $>10$ \\
\hline R29 & $<0.21$ & $<0.03$ & $<0.09$ & & $\ldots$ \\
\hline R30a & $0.77 \pm 0.07$ & $0.39 \pm 0.01$ & $<0.09$ & $0.51 \pm 0.05$ & $>9$ \\
\hline R30b & $1.39 \pm 0.07$ & $0.69 \pm 0.01$ & $<0.09$ & $0.50 \pm 0.03$ & $>15$ \\
\hline R31a & $0.72 \pm 0.07$ & $0.33 \pm 0.01$ & $0.06 \pm 0.03$ & $0.46 \pm 0.03$ & $13 \pm 1$ \\
\hline R31b & $0.55 \pm 0.07$ & $0.24 \pm 0.01$ & $<0.09$ & $0.44 \pm 0.06$ & \\
\hline R32 & $0.55 \pm 0.07$ & $0.29 \pm 0.01$ & $<0.09$ & $0.52 \pm 0.07$ & $>6$ \\
\hline R33 & $0.35 \pm 0.07$ & $0.14 \pm 0.01$ & $<0.09$ & $0.41 \pm 0.09$ & $>4$ \\
\hline R34 & $0.83 \pm 0.07$ & $0.37 \pm 0.01$ & $0.05 \pm 0.03$ & $0.45 \pm 0.04$ & $18 \pm 2$ \\
\hline R35 & $1.42 \pm 0.07$ & $0.30 \pm 0.01$ & $<0.09$ & $0.21 \pm 0.01$ & $>16$ \\
\hline R36a & $0.65 \pm 0.07$ & $0.24 \pm 0.01$ & $0.07 \pm 0.03$ & $0.37 \pm 0.04$ & $9 \pm 1$ \\
\hline R36b & $0.47 \pm 0.07$ & $0.22 \pm 0.01$ & $<0.09$ & $0.47 \pm 0.07$ & $>5$ \\
\hline R37a & $0.79 \pm 0.07$ & $0.15 \pm 0.01$ & $0.06 \pm 0.03$ & $0.19 \pm 0.02$ & $14 \pm 1$ \\
\hline R37b & $0.71 \pm 0.07$ & $0.18 \pm 0.01$ & $<0.09$ & $0.26 \pm 0.03$ & $>8$ \\
\hline R38 & $0.38 \pm 0.07$ & $0.16 \pm 0.01$ & $0.04 \pm 0.03$ & $0.42 \pm 0.08$ & $9 \pm 2$ \\
\hline $\mathrm{R} 39 \mathrm{a}^{\dagger \dagger \dagger}$ & $4.91 \pm 0.07$ & $3.15 \pm 0.01$ & $0.24 \pm 0.03$ & $0.64 \pm 0.01$ & $21 \pm 1$ \\
\hline $\mathrm{R} 39 \mathrm{~b}^{\dagger \dagger \dagger}$ & $1.96 \pm 0.07$ & $0.81 \pm 0.01$ & $0.06 \pm 0.03$ & $0.41 \pm 0.02$ & $31 \pm 2$ \\
\hline
\end{tabular}

Note. - Column 1: These numbers are labeled at the ratio map of Figure 7. Column 2: Peak ${ }^{12} \mathrm{CO}(1-0)$ brightness temperature at an emission region. Column 3: Peak ${ }^{12} \mathrm{CO}(3-2)$ brightness temperature at an emission region. Column 4: Peak ${ }^{13} \mathrm{CO}(3-2)$ brightness temperature at an emission region. Column 5: The ${ }^{12} \mathrm{CO}(3-$ 2)/CO (1-0) brightness temperature ratio. Column 6: The ${ }^{12} \mathrm{CO}(1-0) /{ }^{13} \mathrm{CO}(1-0)$ brightness temperature ratio.; We only show the statistical error in this table. The systematic error of absolute flux calibration is estimated to be $\sim 5 \%$ in band 3 and $\sim 10 \%$ in band 7 . †represents boxes contained the obscured AGN defined by paper I. $\dagger \dagger r e p r e s e n t s$ boxes contained the nuclear starbursts defined by paper I. $\dagger \dagger \dagger$ represents boxes at the overlap region. 
TABLE 7

Peak Brightness Temperature of HCN (4-3) and $\mathrm{HCO}^{+}(4-3)$

\begin{tabular}{|c|c|c|c|}
\hline $\begin{array}{l}\text { ID } \\
\text { (1) }\end{array}$ & $\begin{array}{r}T_{\mathrm{b}, \mathrm{HCN}}(4-3) \\
{[\mathrm{K}]} \\
(2)\end{array}$ & $\begin{array}{r}T_{\mathrm{b}, \mathrm{HCO}}+(4-3) \\
{[\mathrm{K}]} \\
(3)\end{array}$ & $\begin{array}{r}R_{\mathrm{HCN} / \mathrm{HCO}}+ \\
(4)\end{array}$ \\
\hline $\mathrm{S}^{\dagger}$ & $0.32 \pm 0.05$ & $0.27 \pm 0.05$ & $1.18 \pm 0.29$ \\
\hline S1 & $<0.15$ & $0.20 \pm 0.05$ & $<0.75$ \\
\hline $\mathrm{S} 2$ & $<0.15$ & $0.16 \pm 0.05$ & $<0.94$ \\
\hline $\mathrm{S} 3^{\dagger \dagger}$ & $0.20 \pm 0.05$ & $0.65 \pm 0.05$ & $0.31 \pm 0.08$ \\
\hline $\mathrm{S} 4$ & $0.10 \pm 0.05$ & $0.51 \pm 0.05$ & $0.19 \pm 0.10$ \\
\hline $\mathrm{S} 5^{\dagger \dagger}$ & $0.32 \pm 0.05$ & $0.91 \pm 0.05$ & $0.35 \pm 0.06$ \\
\hline S6 & $0.14 \pm 0.05$ & $0.55 \pm 0.05$ & $0.26 \pm 0.09$ \\
\hline S7 & $<0.15$ & $0.24 \pm 0.05$ & $<0.63$ \\
\hline S8 & $<0.15$ & $0.14 \pm 0.05$ & $<1.07$ \\
\hline S9 & $<0.15$ & $0.17 \pm 0.05$ & $<0.88$ \\
\hline S10 & $0.10 \pm 0.05$ & $0.17 \pm 0.05$ & $0.60 \pm 0.34$ \\
\hline $\mathrm{S} 11^{\dagger \dagger \dagger}$ & $<0.15$ & $0.18 \pm 0.05$ & $<0.83$ \\
\hline $\mathrm{S} 12^{\dagger \dagger \dagger}$ & $<0.15$ & $0.24 \pm 0.05$ & $<0.63$ \\
\hline $\mathrm{S} 13$ & $<0.15$ & $0.20 \pm 0.05$ & $<0.75$ \\
\hline S14 & $<0.15$ & $0.23 \pm 0.05$ & $<0.65$ \\
\hline
\end{tabular}

Note. - Column 1: These numbers are labeled at the ratio map of Figure 7. Column 2: Peak HCN (4-3) brightness temperature at an emission region. Column 3: Peak $\mathrm{HCO}^{+}(4-3)$ brightness temperature at an emission region. Column 4: The HCN (4-3)/ $\mathrm{HCO}^{+}(4-3)$ brightness temperature ratio.; We only show the statistical error in this table. The systematic error of absolute flux calibration is estimated to be $\sim 5 \%$ in band 3 and $\sim 10 \%$ in band 7 . †represents boxes contained the obscured AGN defined by paper I. $\dagger \dagger$ represents boxes contained the nuclear starbursts defined by paper I. $\dagger \dagger \dagger$ represents boxes at the overlap region.

TABLE 8

Peak Brightness Temperature of ${ }^{12} \mathrm{CO}(3-2), \mathrm{HCN}(4-3), \mathrm{And}^{\mathrm{HCO}}+(4-3)$

\begin{tabular}{|c|c|c|c|c|c|}
\hline $\begin{array}{l}\text { ID } \\
(1)\end{array}$ & $\begin{array}{r}\text { Peak } T_{\mathrm{b}, \mathrm{CO}}(3-2) \\
{[\mathrm{K}]} \\
(2)\end{array}$ & $\begin{array}{r}\text { Peak } T_{\mathrm{b}, \mathrm{HCN}}(4-3) \\
{[\mathrm{K}]} \\
(3)\end{array}$ & $\begin{array}{r}\text { Peak } T_{\mathrm{b}, \mathrm{HCO}}+(4-3) \\
{[\mathrm{K}]} \\
(4)\end{array}$ & $\begin{array}{r}\text { Peak } R_{\mathrm{HCO}^{+} / \mathrm{CO}} \\
(5)\end{array}$ & $\begin{array}{r}\text { Peak } R_{\mathrm{HCN} / \mathrm{HCO}^{+}} \\
(6)\end{array}$ \\
\hline $\mathrm{E}^{\dagger}$ & $2.94 \pm 0.04$ & $0.06 \pm 0.01$ & $0.04 \pm 0.01$ & $0.014 \pm 0.004$ & $1.55 \pm 0.49$ \\
\hline $\mathrm{E} 1^{\dagger \dagger}$ & $5.82 \pm 0.04$ & $0.11 \pm 0.01$ & $0.32 \pm 0.01$ & $0.056 \pm 0.002$ & $0.36 \pm 0.03$ \\
\hline $\mathrm{E} 2^{\dagger \dagger \dagger}$ & $4.71 \pm 0.04$ & $0.04 \pm 0.01$ & $0.08 \pm 0.01$ & $0.017 \pm 0.002$ & $0.52 \pm 0.14$ \\
\hline
\end{tabular}

Note. - Column 1: These numbers are regions where were convolved to the $1^{\prime \prime} 2 \times 1{ }^{\prime \prime} 0$ resolution (P.A. $=119$ deg.). Column 2: Peak ${ }^{12} \mathrm{CO}(3-2)$ brightness temperature at an emission region. Column 3: Peak HCN (4-3) brightness

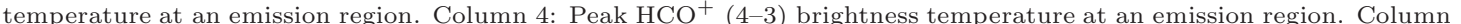
5: The $\mathrm{HCO}^{+}(4-3) / \mathrm{CO}(3-2)$ brightness temperature ratio. Column 6: The $\mathrm{HCN}(4-3) / \mathrm{HCO}^{+}(4-3)$ brightness temperature ratio.; We only show the statistical error in this table. The systematic error of absolute flux calibration is estimated to be $\sim 5 \%$ in band 3 and $\sim 10 \%$ in band 7 . trepresents boxes contained the obscured AGN defined by paper I. $\dagger \dagger$ represents boxes contained the nuclear starbursts defined by paper I. †††represents boxes at the overlap region.

TABLE 9

Parameters Used for RADEX Modelings

\begin{tabular}{lrrrrr}
\hline \hline Case & $\begin{array}{r}T_{\text {kin }} \\
{[\mathrm{K}]}\end{array}$ & $\begin{array}{r}\log n_{\mathrm{H}_{2}} \\
{\left[\mathrm{~cm}^{-3}\right]}\end{array}$ & $\begin{array}{r}\log \mathrm{N}_{\left(\mathrm{H}_{2}\right)} \\
{\left[\mathrm{cm}^{-2}\right]} \\
(4)\end{array}$ & abundance ratio & $\begin{array}{r}\text { box size } \\
{[\mathrm{pc}]} \\
(1)\end{array}$ \\
$(2)$ & $(3)$ & $(5)$ & 800 \\
\hline 1 & $5-300(5)$ & $2-5(0.1)$ & $18-22(0.1)$ & 70 & 800 \\
2 & $5-400(5)$ & $3-7(0.1)$ & $21.2,21.6,21.5$ & $1-10(1)$ & 320 \\
& & & & & \\
\hline
\end{tabular}

Note. - Column 2, 3, 4, 5: Fitting ranges (steps) of $T_{\mathrm{kin}}, n_{\mathrm{H}_{2}}, N\left(\mathrm{H}_{2}\right)$, and abundance ratios. The abundance ratios mean the $[\mathrm{CO}] /\left[{ }^{13} \mathrm{CO}\right]$ and $[\mathrm{HCN}] /\left[\mathrm{HCO}^{+}\right]$ in case 1 and 2 , respectively. We fixed the $[\mathrm{CO}] /\left[{ }^{13} \mathrm{CO}\right]$ in case 1 (Galactic value; Wilson \& Rood 1994) and the $N\left(\mathrm{H}_{2}\right)$ (E0, E1, and E2, respectively) in case 2. Column 6: Box sizes.; We consider the statistical error and the systematic error in these calculations. The systematic error of absolute flux calibration is estimated to be $\sim$ $5 \%$ in band 3 and $\sim 10 \%$ in band 7 . 
TABLE 10

Gas, Dust, and Star-forming Properties (R1 - R39)

\begin{tabular}{|c|c|c|c|c|c|c|c|c|c|}
\hline $\begin{array}{l}\text { ID } \\
\text { (1) }\end{array}$ & 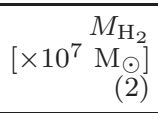 & $\begin{array}{r}L_{\mathrm{Pa \alpha}} \\
{\left[\times 10^{38} \mathrm{erg} \mathrm{s}^{-1}\right]} \\
(3)\end{array}$ & $\begin{array}{r}\mathrm{SFR} \\
{\left[\mathrm{M}_{\odot} \mathrm{yr}^{-1}\right]} \\
(4)\end{array}$ & $\begin{array}{r}\tau_{\text {gas }} \\
{[\mathrm{Gyr}]} \\
(5)\end{array}$ & $\begin{array}{r}S_{340} \\
{[\mathrm{mJy}]} \\
(6)\end{array}$ & $\begin{array}{r}M_{\text {dust }} \\
{\left[\times 10^{4} \mathrm{M}_{\odot}\right]} \\
(7)\end{array}$ & $M_{\mathrm{H}_{2}} /$ & $\overline{M_{\text {dust }}}$ & $\begin{array}{r}M_{\text {ISM }} \\
{\left[\times 10^{7} \mathrm{M}_{\odot}\right]} \\
(9)\end{array}$ \\
\hline $\mathrm{R} 1$ & $3.0 \pm 0.6$ & $<24.6$ & $<0.15$ & $>0.20$ & $<0.51$ & $<17$ & $>$ & 180 & $<3.6$ \\
\hline $\mathrm{R} 2$ & $2.0 \pm 0.4$ & $46.0 \pm 8.2$ & $0.29 \pm 0.05$ & $0.07 \pm 0.02$ & $<0.51$ & $<17$ & $>$ & 119 & $<3.6$ \\
\hline R3 & $5.1 \pm 0.8$ & $11.0 \pm 8.2$ & $0.07 \pm 0.05$ & $0.75 \pm 0.57$ & $<0.51$ & $<17$ & $>$ & 306 & $<3.6$ \\
\hline R4 & $5.0 \pm 0.7$ & $9.3 \pm 8.2$ & $0.06 \pm 0.05$ & $0.88 \pm 0.78$ & $<0.51$ & $<17$ & $>$ & 299 & $<3.6$ \\
\hline R5 & $4.9 \pm 0.7$ & $41.8 \pm 8.2$ & $0.26 \pm 0.05$ & $0.19 \pm 0.05$ & $<0.51$ & $<17$ & $>$ & 293 & $<3.6$ \\
\hline R6 & $5.2 \pm 0.9$ & $60.7 \pm 8.2$ & $0.38 \pm 0.05$ & $0.14 \pm 0.03$ & $<0.51$ & $<17$ & $>$ & 310 & $<3.6$ \\
\hline R7 & $7.5 \pm 0.9$ & $172.1 \pm 8.2$ & $1.07 \pm 0.05$ & $0.07 \pm 0.01$ & $0.61 \pm 0.18$ & $20 \pm 6$ & $371 \pm$ & 118 & $5.2 \pm 1.4$ \\
\hline $\mathrm{R} 8$ & $5.3 \pm 0.8$ & $69.8 \pm 8.2$ & $0.43 \pm 0.05$ & $0.12 \pm 0.02$ & $<0.51$ & $<17$ & $>$ & 314 & $<3.6$ \\
\hline R9 & $13.6 \pm 1.2$ & $125.1 \pm 8.2$ & $0.78 \pm 0.05$ & $0.18 \pm 0.02$ & $<0.51$ & $<17$ & $>$ & 811 & $<3.6$ \\
\hline R10 & $18.0 \pm 1.3$ & $49.0 \pm 8.2$ & $0.30 \pm 0.05$ & $0.59 \pm 0.11$ & $<0.51$ & $<17$ & & 1074 & $<3.6$ \\
\hline R11 & $23.1 \pm 1.6$ & $106.9 \pm 8.2$ & $0.66 \pm 0.05$ & $0.35 \pm 0.04$ & $<0.51$ & $<17$ & & 1376 & $<3.6$ \\
\hline R12 & $6.5 \pm 0.9$ & $25.5 \pm 8.2$ & $0.16 \pm 0.05$ & $0.41 \pm 0.14$ & $<0.51$ & $<17$ & $>$ & 390 & $<3.6$ \\
\hline R13 & $7.4 \pm 1.0$ & $.9 \pm 8.2$ & $0.16 \pm 0.05$ & $0.46 \pm 0.16$ & $<0.51$ & $<17$ & $>$ & 441 & $<3.6$ \\
\hline R14 & $6.7 \pm 0.8$ & $25.5 \pm 8.2$ & $0.16 \pm 0.05$ & $0.42 \pm 0.14$ & $<0.51$ & $<17$ & $>$ & 398 & $<3.6$ \\
\hline R15 & $7.1 \pm 0.9$ & $14.7 \pm 8.2$ & $0.09 \pm 0.05$ & $0.78 \pm 0.44$ & $<0.51$ & $<17$ & $>$ & 425 & $<3.6$ \\
\hline R16 & $7.9 \pm 0.9$ & $15.9 \pm 8.2$ & $0.10 \pm 0.05$ & $0.80 \pm 0.42$ & $<0.51$ & $<17$ & $>$ & 473 & $<3.6$ \\
\hline R17 & $9.1 \pm 1.0$ & $35.2 \pm 8.2$ & $0.22 \pm 0.05$ & $0.42 \pm 0.11$ & $<0.51$ & $<17$ & $>$ & 545 & $<3.6$ \\
\hline $\mathrm{R} 18^{\dagger}$ & $23.5 \pm 1.6$ & $277.4 \pm 8.2$ & $1.72 \pm 0.05$ & $0.14 \pm 0.01$ & $5.57 \pm 0.58$ & $183 \pm 19$ & $128 \pm$ & 16 & $47.1 \pm 4.9$ \\
\hline R19 & $15.7 \pm 1.3$ & $150.8 \pm 8.2$ & $0.94 \pm 0.05$ & $0.17 \pm 0.02$ & $2.70 \pm 0.32$ & $89 \pm 11$ & $177 \pm$ & 25 & $22.9 \pm 2.6$ \\
\hline R20 & $26.0 \pm 1.7$ & $78.1 \pm 8.2$ & $0.48 \pm 0.05$ & $0.54 \pm 0.07$ & $2.40 \pm 0.29$ & $79 \pm 10$ & $329 \pm$ & 46 & $20.3 \pm 2.4$ \\
\hline $\mathrm{R} 21^{\dagger \dagger}$ & $46.9 \pm 2.7$ & $303.2 \pm 8.2$ & $1.88 \pm 0.05$ & $0.25 \pm 0.02$ & $8.55 \pm 0.87$ & $281 \pm 29$ & $167 \pm$ & 19 & $72.3 \pm 7.3$ \\
\hline R22 & $40.7 \pm 2.4$ & $7.1 \pm 8.2$ & $1.22 \pm 0.05$ & $0.33 \pm 0.02$ & $4.08 \pm 0.44$ & $134 \pm 15$ & $303 \pm$ & 37 & $34.5 \pm 3.7$ \\
\hline R23 & $47.8 \pm 2.7$ & $7.5 \pm 8.2$ & $1.35 \pm 0.05$ & $0.35 \pm 0.02$ & \pm 0.40 & $119 \pm 13$ & $403 \pm$ & 50 & $30.5 \pm 3.3$ \\
\hline R24 & $22.9 \pm 1.7$ & $5.5 \pm 8.2$ & $0.90 \pm 0.05$ & $0.25 \pm 0.02$ & $1.28 \pm 0.21$ & $42 \pm 7$ & $543 \pm$ & 99 & $10.8 \pm 1.7$ \\
\hline R25 & $35.8 \pm 2.2$ & $281.2 \pm 8.2$ & $1.74 \pm 0.05$ & $0.21 \pm 0.01$ & $3.10 \pm 0.35$ & $102 \pm 12$ & $351 \pm$ & 46 & $26.2 \pm 2.9$ \\
\hline R26 & $29.0 \pm 1.9$ & $250.1 \pm 8.2$ & $1.55 \pm 0.05$ & $0.19 \pm 0.01$ & $1.66 \pm 0.24$ & $54 \pm 8$ & $533 \pm$ & 84 & $14.0 \pm 1.9$ \\
\hline R27 & $17.7 \pm 1.4$ & $507.8 \pm 8.2$ & $3.15 \pm 0.05$ & $0.06 \pm 0.01$ & $1.42 \pm 0.22$ & $47 \pm 7$ & $379 \pm$ & 66 & $12.0 \pm 1.7$ \\
\hline R28 & $8.2 \pm 1.0$ & $246.3 \pm 8.2$ & $1.53 \pm 0.05$ & $0.05 \pm 0.01$ & $0.73 \pm 0.18$ & $24 \pm 6$ & $344 \pm$ & 97 & $6.1 \pm 1.4$ \\
\hline R29 & $<1.4$ & $7.8 \pm 8.2$ & $0.92 \pm 0.05$ & $<0.02$ & $0.76 \pm 0.19$ & $25 \pm 6$ & $<$ & 55 & $6.4 \pm 1.4$ \\
\hline R30 & $9.1 \pm 1.1$ & $40.2 \pm 8.2$ & $0.25 \pm 0.05$ & $0.36 \pm 0.09$ & $0.79 \pm 0.19$ & $26 \pm 6$ & $348 \pm$ & 92 & $6.7 \pm 1.4$ \\
\hline R31 & $7.0 \pm 1.0$ & $.0 \pm 8.2$ & $0.12 \pm 0.05$ & $0.59 \pm 0.27$ & $<0.51$ & $<17$ & $>$ & 418 & $<3.6$ \\
\hline R32 & $3.3 \pm 0.5$ & $60.3 \pm 8.2$ & $0.37 \pm 0.05$ & $0.09 \pm 0.02$ & $<0.51$ & $<17$ & $>$ & 198 & $<3.6$ \\
\hline R33 & $2.0 \pm 0.4$ & $55.0 \pm 8.2$ & $0.34 \pm 0.05$ & $0.06 \pm 0.0$ & $<0.51$ & $<17$ & $>$ & 122 & $<3.6$ \\
\hline R34 & $4.0 \pm 0.7$ & $10.9 \pm 8.2$ & $0.07 \pm 0.05$ & $0.59 \pm 0.45$ & $<0.51$ & $<17$ & $>$ & 238 & $<3.6$ \\
\hline R35 & $7.6 \pm 0.9$ & $<24.6$ & $<0.15$ & $>0.51$ & $<0.51$ & $<17$ & $>$ & 453 & $<3.6$ \\
\hline R36 & $7.9 \pm 1.1$ & $<24.6$ & $<0.15$ & $>0.52$ & $<0.51$ & $<17$ & & 469 & $<3.6$ \\
\hline R37 & $6.9 \pm 1.0$ & $<24.6$ & $<0.15$ & $>0.46$ & $<0.51$ & $<17$ & $>$ & 410 & $<3.6$ \\
\hline R38 & $3.8 \pm 0.7$ & $15.2 \pm 8.2$ & $0.10 \pm 0.05$ & $0.40 \pm 0.22$ & $<0.51$ & $<17$ & 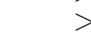 & 225 & $<3.6$ \\
\hline R39 $9^{\dagger \dagger \dagger}$ & $39.1 \pm 2.3$ & $273.6 \pm 8.2$ & $1.70 \pm 0.05$ & $0.23 \pm 0.02$ & $3.50 \pm 0.58$ & $115 \pm 19$ & $339 \pm$ & 60 & $29.6 \pm 3.2$ \\
\hline
\end{tabular}

Note. - Column 2: The molecular gas mass derived using the conversion factor $\alpha_{\mathrm{CO}}=0.8\left(\mathrm{~K} \mathrm{~km} \mathrm{~s}^{-1} \mathrm{pc}^{2}\right)^{-1}$. Column 3: The Pa $\alpha$ flux (Tateuchi et al. 2012). Column 4: The star formation rate derived using the conversion factor SFR $/ L_{\mathrm{Pa} \alpha}=6.2 \times 10^{-41}$ $\left[\operatorname{erg~s}^{-1} /\left(\mathrm{M}_{\odot} \mathrm{yr}^{-1}\right)^{-1}\right]$. Column 5: The gas depletion time $\left(=\Sigma_{\mathrm{H}_{2}} / \Sigma_{\mathrm{SFR}}\right)$. Column 6: The $340 \mathrm{GHz}$ continuum flux. Column 7: The dust mass derived using the equation (7). We adopt the Draine \& Lee (1984) dust model for $\kappa_{340}$ to derive the $M_{\mathrm{dust}}$. Column 9: The ISM mass derived using the equation (8).; We consider the statistical error and the systematic error in this table. The systematic error of absolute flux calibration is estimated to be $\sim 5 \%$ in band 3 and $\sim 10 \%$ in band 7 . †represents boxes contained the obscured AGN defined by paper I. ††represents boxes contained the nuclear starbursts defined by paper I. $\uparrow \dagger \dagger$ represents boxes at the overlap region. 
TABLE 11

Gas, Dust, and Star-Forming Properties (S0 - S14)

\begin{tabular}{|c|c|c|c|c|c|c|c|}
\hline (1) & $\begin{array}{r}M_{\text {dense }} \\
{\left[\times 10^{6} \mathrm{M}_{\odot}\right]} \\
(2)\end{array}$ & $\begin{array}{r}L_{\mathrm{Pa \alpha}} \\
{\left[\times 10^{38} \mathrm{erg} \mathrm{s}^{-1}\right]} \\
(3)\end{array}$ & $\begin{array}{r}\text { SFR } \\
{\left[\mathrm{M}_{\odot} \mathrm{yr}^{-1}\right]} \\
(4)\end{array}$ & $\begin{array}{r}\tau_{\text {gas }} \\
{[\mathrm{Myr}]} \\
(5)\end{array}$ & $\begin{array}{r}S_{340} \\
{[\mathrm{mJy}]} \\
(6)\end{array}$ & $\begin{array}{r}M_{\text {dust }} \\
{\left[\times 10^{4} \mathrm{M}_{\odot}\right]} \\
(7)\end{array}$ & $\begin{array}{r}M_{\text {dense }} / M_{\text {dust }} \\
(8)\end{array}$ \\
\hline $\mathrm{SO}^{\dagger}$ & $38.3 \pm 4.7$ & $322 \pm 12$ & $2.00 \pm 0.07$ & $19.2 \pm 2.5$ & $1.71 \pm 0.18$ & $56.1 \pm 6.0$ & $68 \pm 11$ \\
\hline S1 & $3.2 \pm 1.8$ & $240 \pm 12$ & $1.49 \pm 0.07$ & $2.2 \pm 1.2$ & $0.25 \pm 0.07$ & $8.1 \pm 2.3$ & $40 \pm 25$ \\
\hline $\mathrm{S} 2$ & $<4.3$ & $<36$ & $<0.21$ & $\ldots$ & $0.10 \pm 0.07$ & $3.1 \pm 2.2$ & $<137$ \\
\hline $\mathrm{S} 3^{\dagger \dagger}$ & $13.9 \pm 2.6$ & $663 \pm 12$ & $4.11 \pm 0.07$ & $3.4 \pm 0.6$ & $1.28 \pm 0.14$ & $42.1 \pm 4.7$ & $33 \pm 7$ \\
\hline $\mathrm{S} 4$ & $3.6 \pm 2.2$ & $446 \pm 12$ & $2.76 \pm 0.07$ & $1.3 \pm 0.8$ & $0.63 \pm 0.09$ & $20.6 \pm 3.0$ & $17 \pm 11$ \\
\hline $\mathrm{S} 5^{\dagger \dagger}$ & $23.2 \pm 3.6$ & $606 \pm 12$ & $3.75 \pm 0.07$ & $6.2 \pm 0.7$ & $2.56 \pm 0.26$ & $84.4 \pm 8.7$ & $27 \pm 5$ \\
\hline S6 & $11.5 \pm 2.6$ & $268 \pm 12$ & $1.66 \pm 0.07$ & $6.9 \pm 1.6$ & $0.90 \pm 0.11$ & $29.7 \pm 3.7$ & $39 \pm 10$ \\
\hline S7 & $5.4 \pm 2.3$ & $138 \pm 12$ & $0.85 \pm 0.07$ & $6.4 \pm 2.7$ & $0.40 \pm 0.08$ & $13.0 \pm 2.5$ & $42 \pm 19$ \\
\hline $\mathrm{S} 8$ & $1.8 \pm 1.6$ & $158 \pm 12$ & $0.98 \pm 0.07$ & $1.8 \pm 1.6$ & $0.32 \pm 0.07$ & $10.5 \pm 2.4$ & $17 \pm 16$ \\
\hline S9 & $2.0 \pm 1.8$ & $106 \pm 12$ & $0.66 \pm 0.07$ & $3.1 \pm 2.8$ & $0.28 \pm 0.07$ & $9.2 \pm 2.3$ & $22 \pm 21$ \\
\hline $\mathrm{S} 10$ & $<4.3$ & $203 \pm 12$ & $1.26 \pm 0.07$ & $<3.4$ & $0.34 \pm 0.07$ & $11.3 \pm 2.4$ & $<38$ \\
\hline $\mathrm{S} 11^{\dagger \dagger \dagger}$ & $<4.3$ & $242 \pm 12$ & $1.50 \pm 0.07$ & $<2.9$ & $0.21 \pm 0.07$ & $7.0 \pm 2.3$ & $<61$ \\
\hline $\mathrm{S} 12^{\dagger \dagger \dagger}$ & $<1.4$ & $323 \pm 12$ & $2.00 \pm 0.07$ & $<2.1$ & $0.46 \pm 0.08$ & $15.3 \pm 2.6$ & $<28$ \\
\hline $\mathrm{S} 13$ & $<4.3$ & $291 \pm 12$ & $1.81 \pm 0.07$ & $<2.4$ & $0.46 \pm 0.08$ & $15.2 \pm 2.6$ & $<28$ \\
\hline S14 & $<1.4$ & $219 \pm 12$ & $1.36 \pm 0.07$ & $<3.2$ & $0.27 \pm 0.07$ & $9.0 \pm 2.3$ & $<48$ \\
\hline
\end{tabular}

Note. - Column 2: The molecular gas mass derived using the conversion factor $\alpha_{\mathrm{HCN}}=10 / 0.63\left(\mathrm{~K} \mathrm{~km} \mathrm{~s}^{-1} \mathrm{pc}^{2}\right)^{-1}$. Column 3: The Pa $\alpha$ flux (Tateuchi et al. 2012). Column 4: The star formation rate derived using the conversion factor $\mathrm{SFR} / L_{\mathrm{Pa} \alpha}=6.2 \times 10^{-41}\left[\mathrm{erg} \mathrm{s}^{-1} /\left(\mathrm{M}_{\odot} \mathrm{yr}^{-1}\right)^{-1}\right]$. Column 5: The gas depletion time $\left(=\Sigma_{\text {dense }} / \Sigma_{\mathrm{SFR}}\right)$. Column 6: The $340 \mathrm{GHz}$ continuum flux. Column 7: The dust mass derived using the equation (8). We adopt the Draine \& Lee (1984) dust model for $\kappa_{340}$ to derive the $M_{\text {dust }}$; We consider the statistical error and the systematic error in this table. The systematic error of absolute flux calibration is estimated to be $\sim 5 \%$ in band 3 and $\sim 10 \%$ in band 7 . †represents boxes contained the obscured AGN defined by paper I. ††represents boxes contained the nuclear starbursts defined by paper I. †† †represents boxes at the overlap region. 
TABLE 12

RADEX Results of CASE 1

\begin{tabular}{|c|c|c|c|c|c|c|c|}
\hline \multirow{2}{*}{$\begin{array}{l}\text { ID } \\
\text { (1) } \\
\end{array}$} & \multicolumn{2}{|c|}{$T_{\text {kin }}$} & \multicolumn{2}{|c|}{$\log n_{\mathrm{H}_{2}}$} & \multicolumn{2}{|c|}{$\log N\left(\mathrm{H}_{2}\right)$} & \multirow{2}{*}{$\begin{array}{r}\min . \chi^{2} \\
(8)\end{array}$} \\
\hline & $\begin{array}{r}\chi^{2}<7.81 \\
{[\mathrm{~K}]} \\
(2)\end{array}$ & $\begin{array}{r}\min . \chi^{2} \\
{[\mathrm{~K}]} \\
(3)\end{array}$ & $\begin{array}{r}\chi^{2}<7.81 \\
{\left[\mathrm{~cm}^{-3}\right]} \\
(4)\end{array}$ & $\begin{array}{r}\min . \chi^{2} \\
{\left[\mathrm{~cm}^{-3}\right]} \\
(5)\end{array}$ & $\begin{array}{r}\chi^{2}<7.81 \\
{\left[\mathrm{~cm}^{-2}\right]} \\
(6)\end{array}$ & $\begin{array}{r}\min . \chi^{2} \\
{\left[\mathrm{~cm}^{-2}\right]} \\
(7)\end{array}$ & \\
\hline R5 & $>5$ & 90 & $<3.7$ & 2.4 & $20.4-21.2$ & 20.7 & 0.0001508 \\
\hline $\mathrm{R} 7 \mathrm{~b}$ & $5-120$ & 25 & $>2.8$ & 4.1 & $>20.8$ & 21.4 & 0.0008348 \\
\hline $\mathrm{R} 8 \mathrm{~b}$ & $5-30$ & 25 & $<2.4$ & 2.0 & $20.9-21.8$ & 21.3 & 0.04646 \\
\hline $\mathrm{R} 9 \mathrm{~b}$ & $>5$ & 50 & $2.2-4.0$ & 3.0 & $20.1-20.7$ & 20.4 & 0.004822 \\
\hline R10a & $5-120$ & 65 & $<3.3$ & 2.1 & $20.4-21.8$ & 20.9 & 0.001529 \\
\hline R10b & $5-20$ & 10 & $3.6-4.6$ & 4.4 & $19.9-20.4$ & 20.1 & 0.00948 \\
\hline R11a & $>20$ & 75 & $<3.2$ & 2.5 & $20.5-21.0$ & 20.8 & 0.000315 \\
\hline R11b & $\ldots$ & 15 & $2.6-4.6$ & 3.9 & $<20.4$ & 18.1 & 0.001707 \\
\hline R13a & $5-200$ & 85 & $<3.5$ & 2.2 & $20.3-21.4$ & 20.7 & 0.002488 \\
\hline R14 & $5-200$ & 15 & $<3.4$ & 3.2 & $20.2-20.6$ & 20.4 & 0.004464 \\
\hline $\mathrm{R} 15$ & $5-140$ & 35 & $<3.4$ & 2.6 & $20.2-20.8$ & 20.5 & 0.001343 \\
\hline R16 & $5-30$ & 15 & $2.4-3.2$ & 2.8 & $20.6-21.0$ & 20.8 & 0.03139 \\
\hline $\mathrm{R} 17$ & $5-180$ & 135 & $<3.6$ & 2.0 & $19.6-21.2$ & 20.2 & 0.003854 \\
\hline $\mathrm{R} 18^{\dagger}$ & $>35$ & 180 & $2.4-3.3$ & 2.7 & $20.6-21.0$ & 20.8 & 0.000186 \\
\hline R19a & $>25$ & 85 & $<3.2$ & 2.5 & $20.9-21.5$ & 21.2 & 0.0001408 \\
\hline R19b & $>20$ & 100 & $2.2-3.5$ & 2.8 & $20.5-21.0$ & 20.8 & 0.002962 \\
\hline R20a & $>30$ & 100 & $2.7-3.6$ & 3.1 & $20.5-21.1$ & 20.8 & 0.001267 \\
\hline $\mathrm{R} 21 \mathrm{a}^{\dagger \dagger}$ & $25-90$ & 50 & $3.4-5.0$ & 3.7 & $20.8-21.5$ & 21.1 & 0.01197 \\
\hline $\mathrm{R} 22 \mathrm{a}$ & $>25$ & 70 & $2.6-3.6$ & 3.1 & $20.5-20.9$ & 20.7 & 0.01047 \\
\hline $\mathrm{R} 22 \mathrm{~b}$ & $>90$ & 265 & $2.8-3.3$ & 3.0 & $21.0-21.6$ & 21.3 & 0.00147 \\
\hline R23a & $>40$ & 140 & $2.7-3.5$ & 3.1 & $20.6-21.1$ & 20.9 & 0.001661 \\
\hline $\mathrm{R} 23 \mathrm{~b}$ & $>35$ & 140 & $2.5-3.5$ & 2.9 & $20.5-21.0$ & 20.8 & 0.002198 \\
\hline $\mathrm{R} 24 \mathrm{a}$ & $>5$ & 160 & $<4.9$ & 2.4 & $20.2-21.3$ & 20.6 & 0.000268 \\
\hline R24b & $>15$ & 275 & $2.5-3.6$ & 2.8 & $20.6-21.4$ & 21.0 & 0.001229 \\
\hline $\mathrm{R} 24 \mathrm{c}$ & $>5$ & 115 & $<3.9$ & 2.5 & $20.0-20.8$ & 20.4 & 0.0001756 \\
\hline $\mathrm{R} 25 \mathrm{a}$ & $5-40$ & 20 & $3.5-5.0$ & 3.9 & $20.5-20.8$ & 20.7 & 0.002611 \\
\hline $\mathrm{R} 25 \mathrm{~b}$ & $>5$ & 285 & $<3.9$ & 2.2 & $20.2-21.0$ & 20.4 & 0.0001641 \\
\hline $\mathrm{R} 26 \mathrm{a}$ & $>40$ & 265 & $2.6-3.4$ & 2.8 & $20.5-21.1$ & 20.8 & 0.0002885 \\
\hline $\mathrm{R} 26 \mathrm{~b}$ & $10-100$ & 50 & $<3.3$ & 2.2 & $20.6-21.4$ & 20.9 & 0.00003488 \\
\hline $\mathrm{R} 27 \mathrm{a}$ & $>5$ & 255 & $>2.9$ & 3.0 & $<21.6$ & 20.6 & 0.0004384 \\
\hline $\mathrm{R} 27 \mathrm{~b}$ & $>50$ & 160 & $<2.6$ & 2.0 & $21.0-21.7$ & 21.3 & 0.001546 \\
\hline $\mathrm{R} 28 \mathrm{a}$ & $>5$ & 110 & $<3.9$ & 2.5 & 20.621 .6 & 21.0 & 0.00007702 \\
\hline R31a & $>20$ & 115 & $<3.0$ & 2.0 & $20.8-22.0$ & 21.3 & 0.001847 \\
\hline R34 & $>5$ & 130 & $<3.4$ & 2.4 & $20.5-21.6$ & 20.9 & 0.0006237 \\
\hline R36a & $10-40$ & 30 & $<2.5$ & 2.0 & $>21.1$ & 21.6 & 0.00002201 \\
\hline R37a & $5-30$ & 25 & $<2.7$ & 2.0 & $20.7-21.8$ & 21.1 & 0.03561 \\
\hline R38 & $>5$ & 35 & $<3.7$ & 2.5 & $>20.8$ & 21.2 & 0.0005419 \\
\hline $\mathrm{R} 39 \mathrm{a}^{\dagger \dagger \dagger}$ & $>50$ & 95 & $2.7-3.4$ & 3.1 & $20.9-21.3$ & 21.1 & 0.006462 \\
\hline $\mathrm{R} 39 \mathrm{~b}^{\dagger \dagger \dagger}$ & $>5$ & 175 & $2.3-4.1$ & 2.6 & $20.0-20.9$ & 20.5 & 0.005283 \\
\hline
\end{tabular}

Note. - Column 2 - 7: RADEX parameters noted above. Column 8: Value of $\chi^{2}$ associated with the fit. Note that for each position, (3), (5), and (7) are the best-fit parameters and (2), (4), and (6) are estimated within a confidence of $95 \%$.; We consider the statistical error and the systematic error in this table. The systematic error of absolute flux calibration is estimated to be $\sim 5 \%$ in band 3 and $\sim 10 \%$ in band 7. †represents boxes contained the obscured AGN defined by paper I. ††represents boxes contained the nuclear starbursts defined by paper I. $\dagger \dagger \dagger$ represents boxes at the overlap region.

TABLE 13

RADEX RESUlts OF CASE 2

\begin{tabular}{|c|c|c|c|c|c|c|c|c|}
\hline \multirow{2}{*}{$\begin{array}{l}\text { ID } \\
\text { (1) }\end{array}$} & \multirow{2}{*}{$\begin{array}{r}\log N\left(\mathrm{H}_{2}\right) \\
{\left[\mathrm{cm}^{-2}\right]} \\
(2)\end{array}$} & \multicolumn{2}{|c|}{$T_{\text {kin }}$} & \multicolumn{2}{|c|}{$\log n_{\mathrm{H}_{2}}$} & \multicolumn{2}{|c|}{ 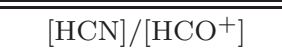 } & \multirow{2}{*}{$\overline{\min .} \chi^{2}$} \\
\hline & & $\begin{array}{r}\chi^{2}<7.81 \\
{[\mathrm{~K}]} \\
(3)\end{array}$ & $\begin{array}{r}\min . \chi^{2} \\
{[\mathrm{~K}]} \\
(4)\end{array}$ & $\begin{array}{r}\chi^{2}<7.81 \\
{\left[\mathrm{~cm}^{-3}\right]} \\
(5)\end{array}$ & $\begin{array}{r}\min . \chi^{2} \\
{\left[\mathrm{~cm}^{-3}\right]} \\
(6)\end{array}$ & $\begin{array}{r}\chi^{2}<0.35 \\
{\left[\mathrm{~cm}^{-2}\right]} \\
(7)\end{array}$ & $\begin{array}{r}\min . \chi^{2} \\
{\left[\mathrm{~cm}^{-2}\right]} \\
(8)\end{array}$ & \\
\hline $\mathrm{E}^{\dagger}$ & 21.2 & $>100$ & 270 & $5.0-5.4$ & 5.3 & $>5$ & 8 & 0.00563 \\
\hline $\mathrm{E} 1^{\dagger \dagger}$ & 21.6 & $40-100$ & 70 & $5.6-5.9$ & 5.8 & $<4$ & 2 & 0.01073 \\
\hline $\mathrm{E} 2^{\dagger \dagger \dagger}$ & 21.5 & $5-90$ & 40 & $5.0-5.6$ & 5.1 & $1-9$ & 2 & 0.0003303 \\
\hline
\end{tabular}

Note. - Column 2: Adopted $N\left(\mathrm{H}_{2}\right)$ which derived from $\mathrm{CO}(1-0)$ data and $X_{\mathrm{CO}}$. Column 3 - 8: RADEX parameters noted above. Column 9: Value of $\chi^{2}$ associated with the fit. Note that for each position, (4), (6), and (8) are the best-fit parameters and (3), (5), and (7) are estimated within the confidence level of $95 \%$ for $T_{\text {kin }}$ and $n_{\mathrm{H}_{2}}$, and $95 \%(2 \sigma)$ for $[\mathrm{HCN}] /\left[\mathrm{HCO}^{+}\right]$.; We consider the statistical error and the systematic error in this table. The systematic error of absolute flux calibration is estimated to be $\sim 5 \%$ in band 3 and $\sim 10 \%$ in band 7. †represents boxes contained the obscured AGN defined by paper I. ††represents boxes contained the nuclear starbursts defined by paper I. $\dagger \dagger \dagger$ represents boxes at the overlap region. 
TABLE 14

Gas Properties under the LTE Assumption

\begin{tabular}{|c|c|c|c|c|c|}
\hline $\begin{array}{l}\text { ID } \\
\text { (1) }\end{array}$ & $\begin{array}{r}\Phi_{\mathrm{A}}\left(=\mathrm{T}_{\mathrm{b}, \mathrm{CO}(1-0)} / \mathrm{T}_{\mathrm{kin}}\right) \\
(2)\end{array}$ & $\begin{array}{r}\text { Adopted } \Phi_{\mathrm{A}} \\
(3)\end{array}$ & $\begin{array}{l}T_{\mathrm{ex}} \\
{[\mathrm{K}]} \\
(4)\end{array}$ & $\begin{array}{r}\tau_{\mathrm{CO}(1-0)} \\
(5)\end{array}$ & $\begin{array}{r}\tau_{13} \mathrm{CO}(1-0) \\
(6)\end{array}$ \\
\hline R5 & $<0.24$ & 0.10 & $15.3 \pm 0.9$ & 3.59 & 0.14 \\
\hline $\mathrm{R} 7 \mathrm{~b}$ & $<0.09$ & 0.09 & $8.3 \pm 0.8$ & 3.97 & 0.02 \\
\hline $\mathrm{R} 8 \mathrm{~b}$ & $0.03-0.19$ & 0.10 & $13.0 \pm 0.9$ & 25.28 & 1.57 \\
\hline $\mathrm{R} 9 \mathrm{~b}$ & $<0.52$ & 0.10 & $29.5 \pm 1.5$ & 1.27 & 0.02 \\
\hline R10a & $0.01-0.16$ & 0.10 & $11.4 \pm 0.8$ & 6.72 & 0.35 \\
\hline R10b & $0.14-0.56$ & 0.14 & $23.5 \pm 1.1$ & 1.24 & 0.03 \\
\hline R11a & $<0.11$ & 0.10 & $24.6 \pm 1.3$ & 3.88 & 0.19 \\
\hline R11b & $\ldots$ & 0.10 & $36.9 \pm 1.8$ & 0.01 & 0.01 \\
\hline R13a & $0.01-0.22$ & 0.10 & $14.6 \pm 0.9$ & 6.87 & 0.37 \\
\hline R14 & $0.01-0.32$ & 0.10 & $19.6 \pm 1.1$ & 5.89 & 0.35 \\
\hline $\mathrm{R} 15$ & $0.01-0.35$ & 0.10 & $21.2 \pm 1.1$ & 6.44 & 0.27 \\
\hline R16 & $0.05-0.28$ & 0.10 & $17.2 \pm 1.0$ & 9.00 & 0.31 \\
\hline R17 & $0.01-0.33$ & 0.10 & $19.9 \pm 1.1$ & 2.59 & 0.06 \\
\hline $\mathrm{R} 18^{\dagger}$ & $<0.10$ & 0.10 & $38.7 \pm 1.9$ & 1.34 & 0.03 \\
\hline R19a & $<0.04$ & 0.04 & $28.7 \pm 2.2$ & 6.99 & 0.76 \\
\hline R19b & $<0.10$ & 0.10 & $23.4 \pm 1.2$ & 1.68 & 0.05 \\
\hline R20a & $<0.11$ & 0.11 & $36.8 \pm 1.8$ & 0.57 & 0.01 \\
\hline $\mathrm{R}_{21 \mathrm{a}^{\dagger \dagger}}$ & $0.06-0.22$ & 0.10 & $58.8 \pm 2.9$ & 2.75 & 0.03 \\
\hline $\mathrm{R} 22 \mathrm{a}$ & $<0.14$ & 0.10 & $39.5 \pm 1.9$ & 0.90 & 0.01 \\
\hline $\mathrm{R} 22 \mathrm{~b}$ & $<0.04$ & 0.04 & $99.3 \pm 5.1$ & 0.42 & 0.01 \\
\hline $\mathrm{R} 23 \mathrm{a}$ & $<0.10$ & 0.10 & $45.2 \pm 2.2$ & 0.61 & 0.01 \\
\hline $\mathrm{R} 23 \mathrm{~b}$ & $<0.10$ & 0.10 & $37.4 \pm 1.8$ & 0.87 & 0.01 \\
\hline R24a & $<0.25$ & 0.10 & $15.7 \pm 0.9$ & 4.00 & 0.21 \\
\hline $\mathrm{R} 24 \mathrm{~b}$ & $<0.11$ & 0.10 & $19.5 \pm 1.1$ & 1.02 & 0.01 \\
\hline $\mathrm{R} 24 \mathrm{c}$ & $<0.36$ & 0.10 & $21.3 \pm 1.1$ & 1.83 & 0.05 \\
\hline $\mathrm{R} 25 \mathrm{a}$ & $0.12-0.98$ & 0.12 & $44.3 \pm 2.1$ & 2.85 & 0.03 \\
\hline $\mathrm{R} 25 \mathrm{~b}$ & $<0.35$ & 0.10 & $21.2 \pm 1.1$ & 2.16 & 0.07 \\
\hline $\mathrm{R} 26 \mathrm{a}$ & $<0.11$ & 0.10 & $48.4 \pm 2.4$ & 1.56 & 0.02 \\
\hline $\mathrm{R} 26 \mathrm{~b}$ & $0.01-0.11$ & 0.10 & $14.1 \pm 0.9$ & 6.33 & 0.30 \\
\hline $\mathrm{R} 27 \mathrm{a}$ & $<0.39$ & 0.10 & $23.1 \pm 1.2$ & 0.09 & 0.01 \\
\hline $\mathrm{R} 27 \mathrm{~b}$ & $<0.03$ & 0.03 & $54.5 \pm 3.5$ & 9.17 & 0.92 \\
\hline R28a & $<0.15$ & 0.10 & $11.0 \pm 0.8$ & 4.21 & 0.31 \\
\hline R31a & $<0.04$ & 0.04 & $21.5 \pm 2.0$ & 4.61 & 0.09 \\
\hline R34 & $<0.17$ & 0.10 & $11.7 \pm 0.8$ & 4.23 & 0.29 \\
\hline R36a & $0.02-0.07$ & 0.07 & $12.7 \pm 1.1$ & 20.17 & 1.53 \\
\hline R37a & $0.03-0.16$ & 0.10 & $11.3 \pm 0.8$ & 19.92 & 1.04 \\
\hline R38 & $<0.08$ & 0.08 & $8.0 \pm 0.9$ & 10.07 & 0.67 \\
\hline R39a ${ }^{\dagger \dagger \dagger}$ & $<0.10$ & 0.10 & $52.6 \pm 2.6$ & 1.42 & 0.01 \\
\hline $\mathrm{R} 39 \mathrm{~b}^{\dagger \dagger \dagger}$ & $<0.39$ & 0.10 & $23.1 \pm 1.2$ & 1.29 & 0.02 \\
\hline
\end{tabular}

Note. - Column 2: The beam filling factor estimated from RADEX modeling and the brightness temperature. Column 3: The adopted beam filling factor. Column 4: The excitation temperature of a spectral line calculated from the equation (3). Column 5 and 6 : the optical depth of ${ }^{12} \mathrm{CO}(1-0)$ and ${ }^{13} \mathrm{CO}(1-0)$ emission from RADEX modeling, respectively. Column 7: The total column density of ${ }^{13} \mathrm{CO}$.; We consider the statistical error and the systematic error in this table. The systematic error of absolute flux calibration is estimated to be $\sim 5 \%$ in band 3 and $\sim 10 \%$ in band 7 . †represents boxes contained the obscured AGN defined by paper I. ††represents boxes contained the nuclear starbursts defined by paper I. †††represents boxes at the overlap region. 
TABLE 15

Properties of CS, $\mathrm{CH}_{3} \mathrm{OH}$, and CN

\begin{tabular}{|c|c|c|c|c|c|}
\hline $\begin{array}{l}\text { Molecule } \\
\text { (1) }\end{array}$ & $\begin{array}{l}\text { ID } \\
(2) \\
\end{array}$ & $\begin{array}{c}T_{\mathrm{b}} \\
{[\mathrm{K}]} \\
(3) \\
\end{array}$ & $\begin{array}{r}\Delta v \\
{\left[\mathrm{~km} \mathrm{~s}^{-1}\right]} \\
(4)\end{array}$ & $\begin{array}{r}N_{\mathrm{X}} \\
{\left[\mathrm{cm}^{-2}\right]} \\
(5)\end{array}$ & $\begin{array}{r}{[X] /\left[\mathrm{H}_{2}\right]} \\
(6) \\
\end{array}$ \\
\hline \multirow[t]{3}{*}{ CS } & $\mathrm{R} 18^{\dagger}$ & $<0.04$ & $\cdots$ & $<4.0 \times 10^{11}$ & $<6.3 \times 10^{-10}$ \\
\hline & $\mathrm{R} 21 \mathrm{a}^{\dagger \dagger}$ & $<0.04$ & $\cdots$ & $<5.6 \times 10^{11}$ & $<4.5 \times 10^{-10}$ \\
\hline & $\mathrm{R} 39 \mathrm{a}^{\dagger \dagger \dagger}$ & $0.13 \pm 0.04$ & $44 \pm 11$ & $1.6 \times 10^{12}$ & $1.3 \times 10^{-9}$ \\
\hline \multirow[t]{3}{*}{$\mathrm{CH}_{3} \mathrm{OH}$} & $\mathrm{R} 18^{\dagger}$ & $<0.04$ & $\ldots$ & $<1.9 \times 10^{12}$ & $<2.9 \times 10^{-9}$ \\
\hline & $\mathrm{R} 21 \mathrm{a}^{\dagger \dagger}$ & $<0.04$ & $\cdots$ & $<2.6 \times 10^{12}$ & $<2.1 \times 10^{-9}$ \\
\hline & $\mathrm{R} 39 \mathrm{a}^{\dagger \dagger \dagger}$ & $0.17 \pm 0.04$ & $43 \pm 5$ & $1.0 \times 10^{13}$ & $8.1 \times 10^{-9}$ \\
\hline \multirow[t]{3}{*}{$\mathrm{CN}$} & $\mathrm{R} 18^{\dagger}$ & $0.23 \pm 0.04$ & $63 \pm 5$ & $2.4 \times 10^{12}$ & $3.8 \times 10^{-9}$ \\
\hline & $\mathrm{R} 21 \mathrm{a}^{\dagger \dagger}$ & $0.15 \pm 0.04$ & $66 \pm 3$ & $2.2 \times 10^{12}$ & $1.8 \times 10^{-9}$ \\
\hline & $\mathrm{R} 39 \mathrm{a}^{\dagger \dagger \dagger}$ & $0.14 \pm 0.04$ & $49 \pm 9$ & $1.4 \times 10^{12}$ & $1.1 \times 10^{-9}$ \\
\hline
\end{tabular}

Note. - Column 1: The molecular line considered. Column 3: Peak brightness temperature of considered line. Column 4: Line width of considered line. Column 5: the derived total box-averaged column density of this species. Column 6: Fractional abundance relative to $\mathrm{H}_{2}$ of this species.; We consider the statistical error and the systematic error in this table. The systematic error of absolute flux calibration is estimated to be $\sim 5 \%$ in band 3 and $\sim 10 \%$ in band 7 . †represents boxes contained the obscured AGN defined by paper I. ††represents boxes contained the nuclear starbursts defined by paper I. †††represents boxes at the overlap region. 
APPENDIX

A. IMAGES AND SPECTRA OF VV114

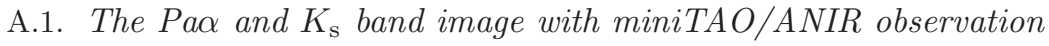
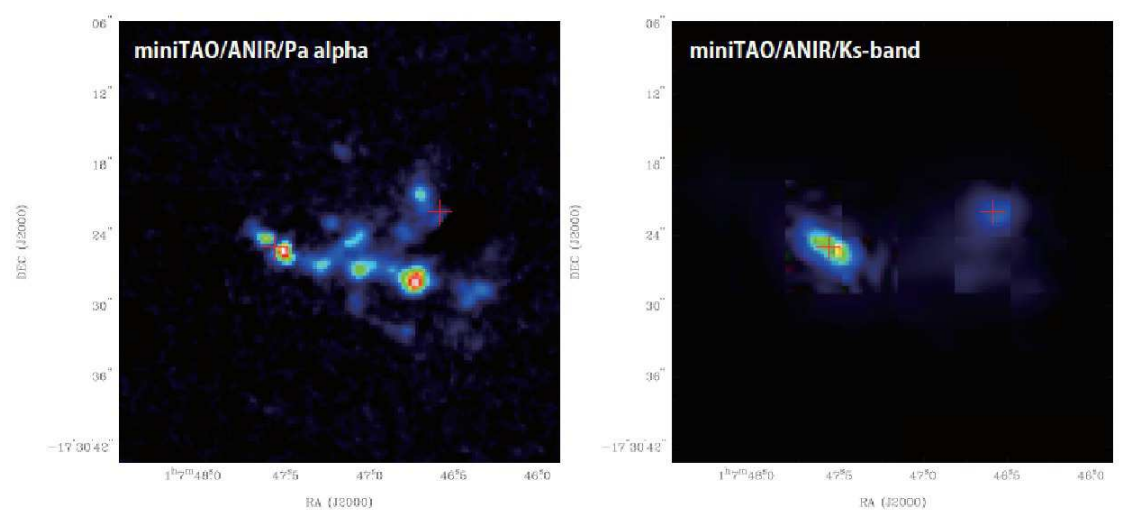

FIG. 14.- The Pa $\alpha$ and $K_{\mathrm{S}}$ band images of VV 114 with miniTAO/ANIR observation (Tateuchi et al. 2012). 


\section{A.2. Channel maps of each line emission}

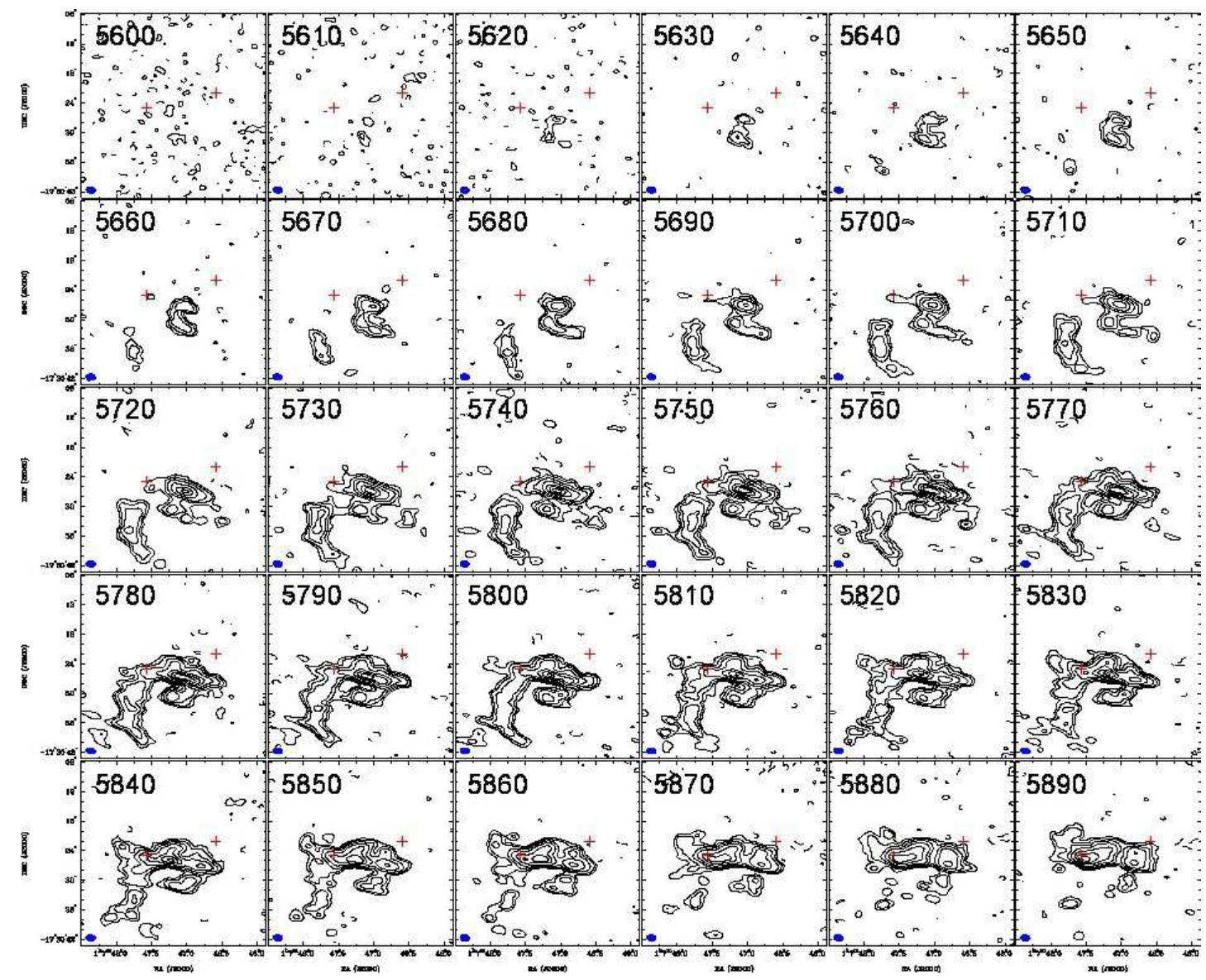

FIG. 15.- The velocity channel maps of the ${ }^{12} \mathrm{CO}(1-0)$ line emission of VV 114. Two crosses in each channel show the positions of the nuclei defined by the peak positions of the Ks-band observation (Tateuchi et al. 2012). The velocity width of each channel is $10 \mathrm{~km} \mathrm{~s}{ }^{-1}$ The beam size is plotted in the bottom-left corner of each channel. The contours represent flux intensity levels: $-4.6,4.6,9.2,18.4,36.8$, $73.6,110.4$ and 147.2 mJy beam ${ }^{-1}$. 


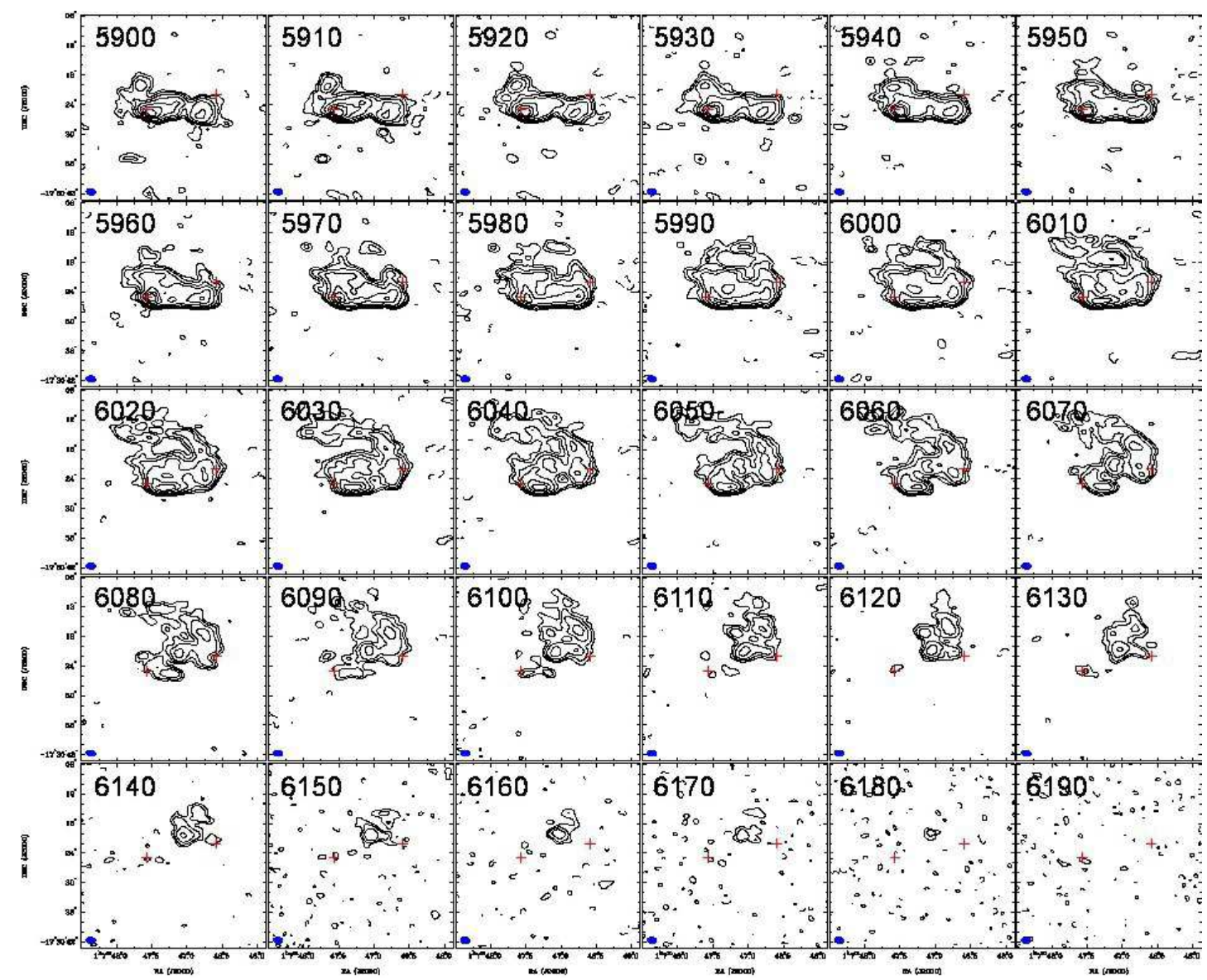

FIG. 15.- Continuied. 


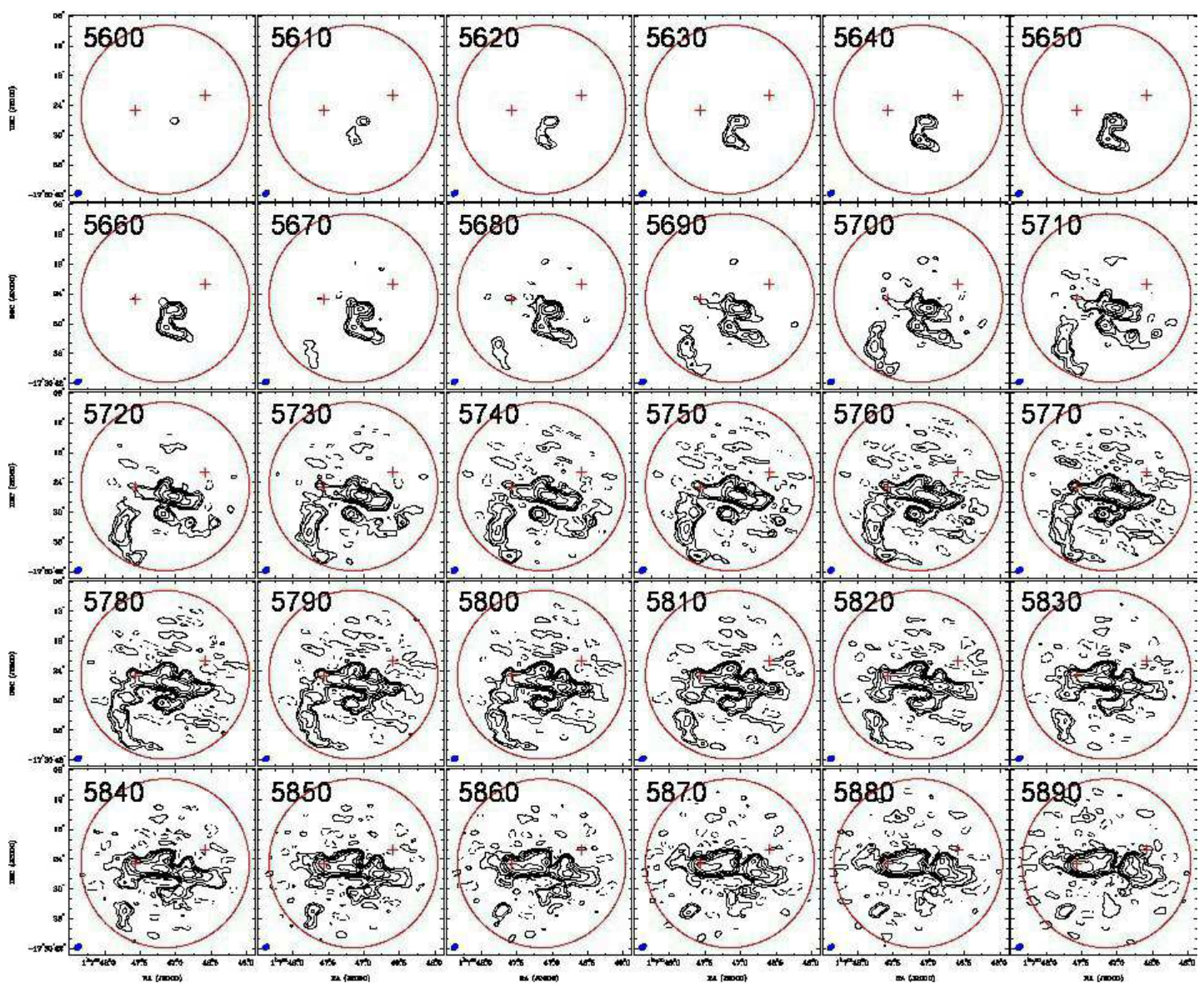

Fig. 16. - The same as Figure 14 but for the CO (3-2) line emission of VV 114. The approximate field of view of ALMA 7-point mosaic at this frequency is indicated by the large red circle. The contours represent flux intensity levels: $-12.6,12.6,25.2,50.4,100.8,201.6$, and $403.2 \mathrm{mJy}^{\text {beam }^{-1}}$. 


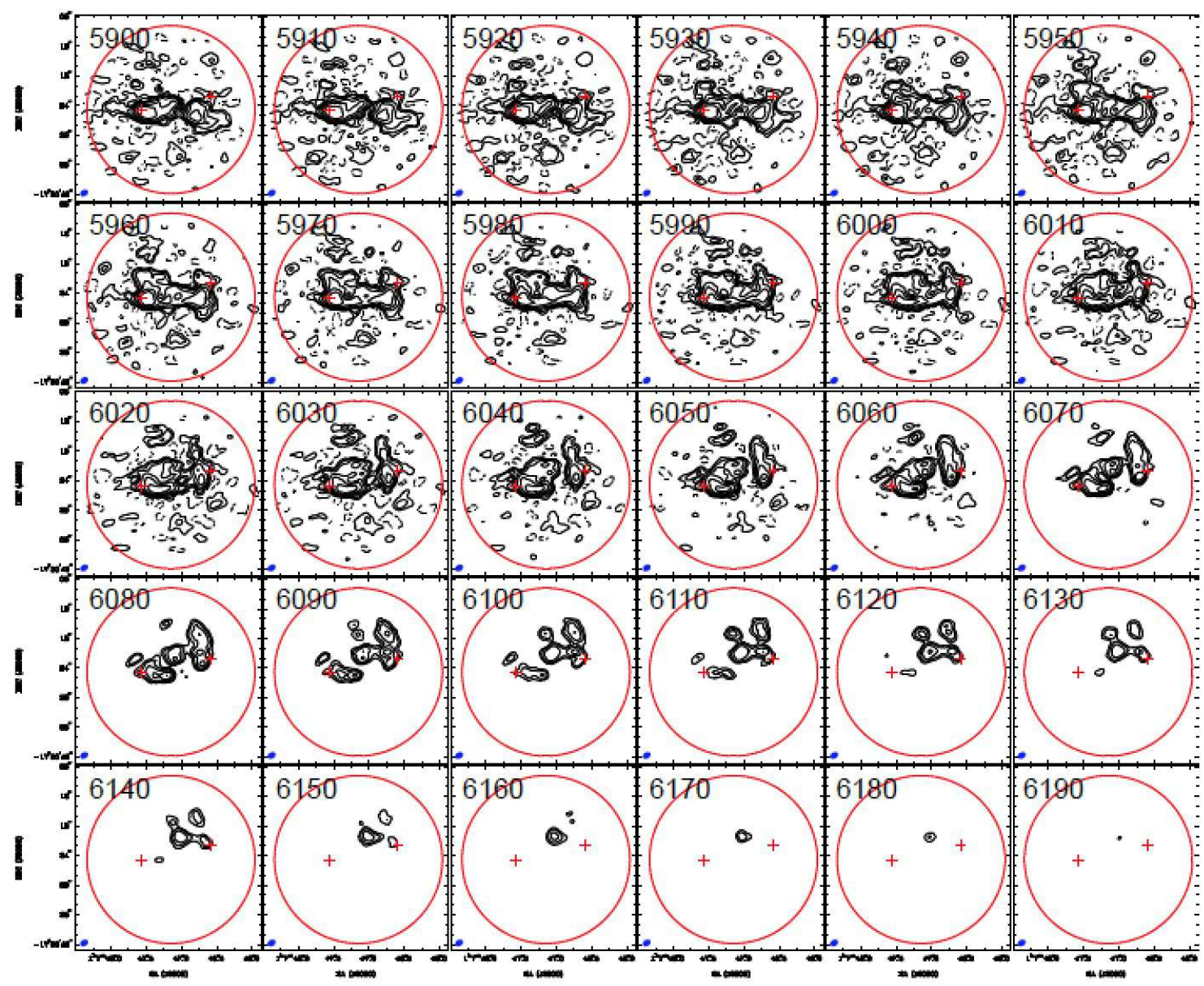

Fig. 16.- Continuied. 


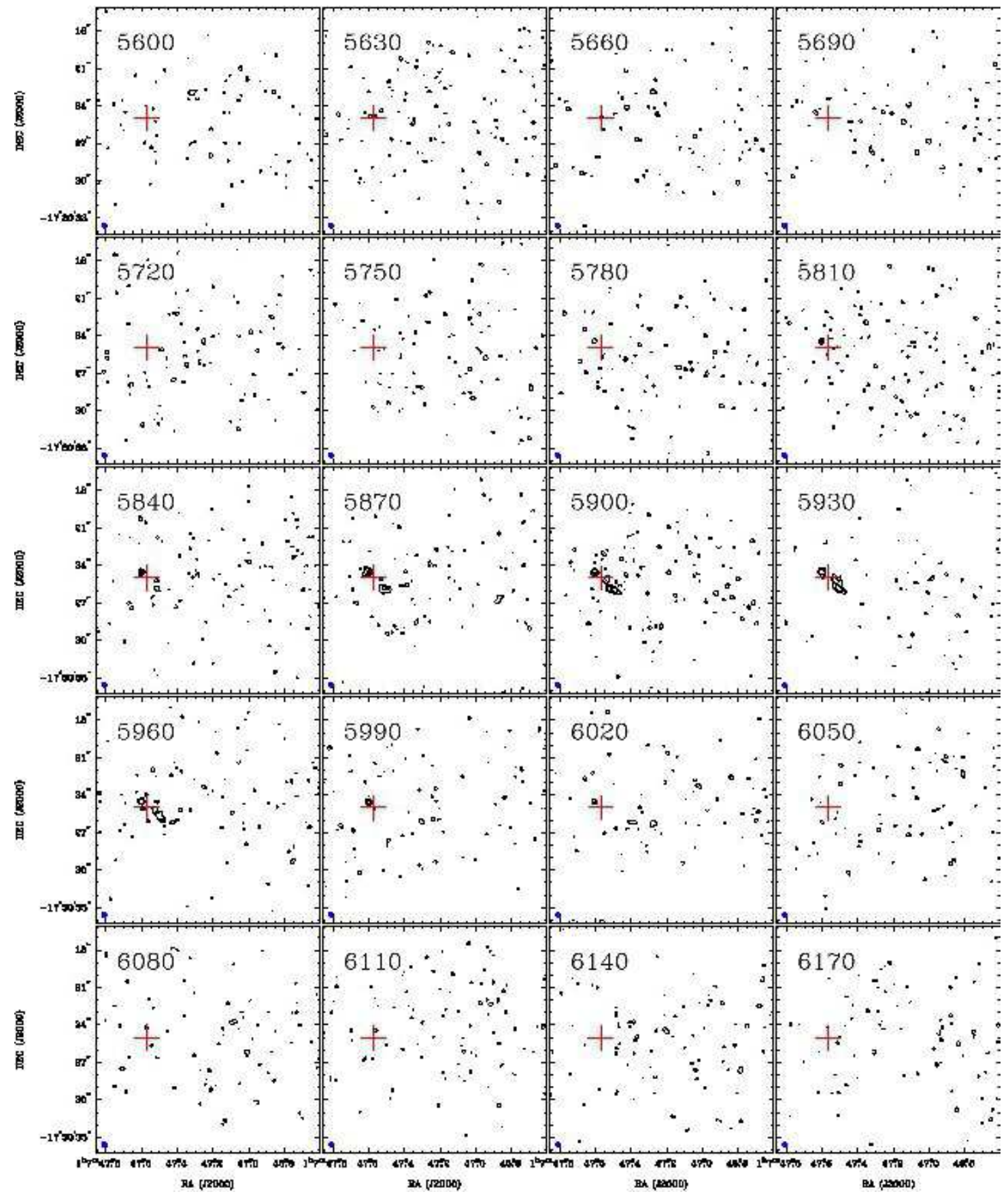

FIG. 17. - The same as Figure 14 but for the HCN (4-3) line emission of VV 114. The velocity width of each channel is $30 \mathrm{~km} \mathrm{~s}{ }^{-1}$. The cross in each channel shows the position of the eastern nucleus defined by the peak position of the Ks-band observation (Tateuchi et al. 2012). The contours represent flux intensity levels: $-2.4,2.4,4.8,9.6$, and $19.2 \mathrm{mJy}_{\mathrm{beam}}{ }^{-1}$. 


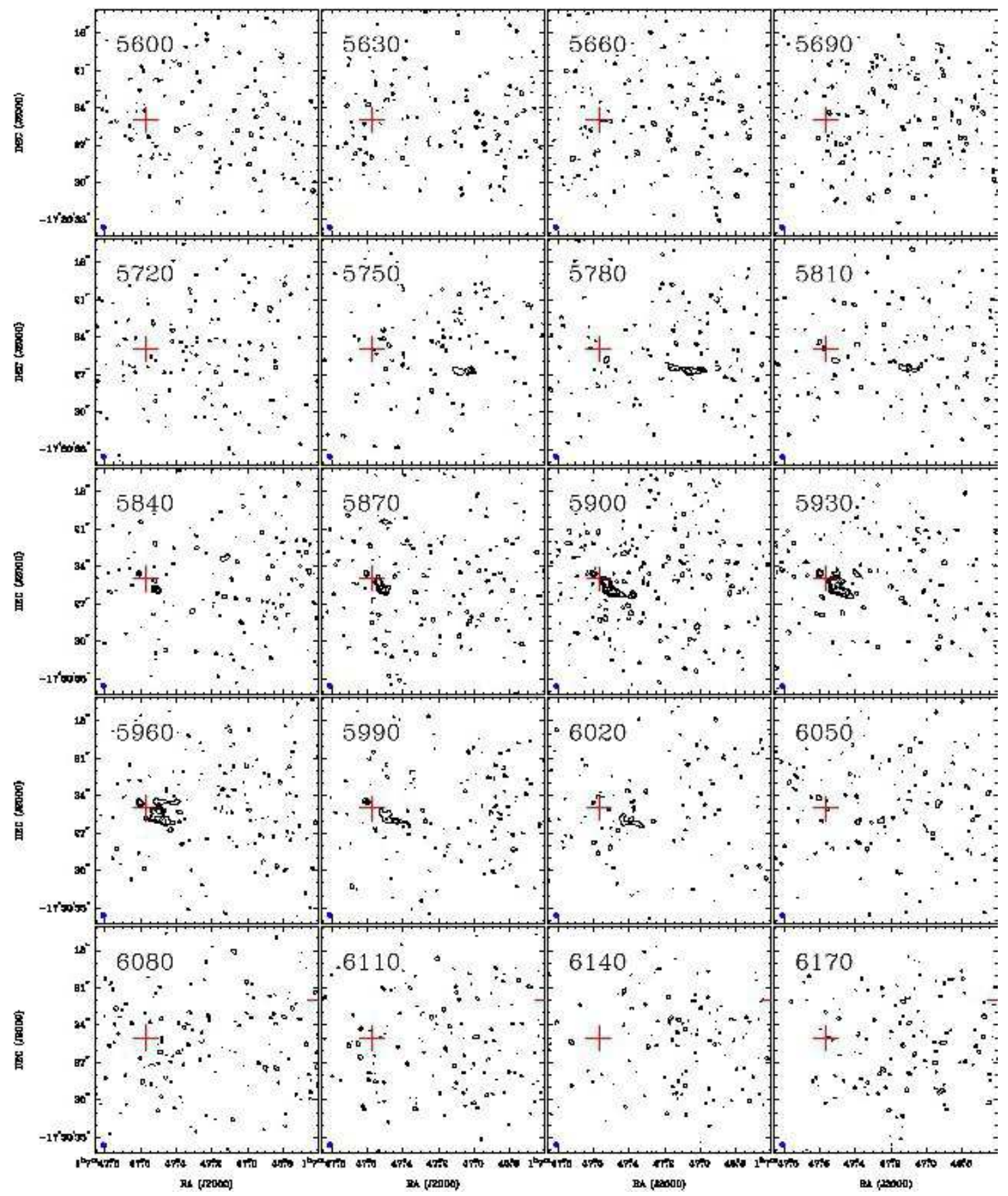

FIg. 18. - The same as Figure 14 but for the $\mathrm{HCO}^{+}(4-3)$ line emission of VV 114. The velocity width of each channel is $30 \mathrm{~km} \mathrm{~s} \mathrm{~s}^{-1}$. The cross in each channel shows the position of the eastern nucleus defined by the peak position of the Ks-band observation (Tateuchi et al. 2012). The contours represent flux intensity levels: $-2.4,2.4,4.8,9.6$, and $19.2 \mathrm{mJy}_{\text {beam }}{ }^{-1}$. 


\section{A.3. Box-summed spectra of each line emission}
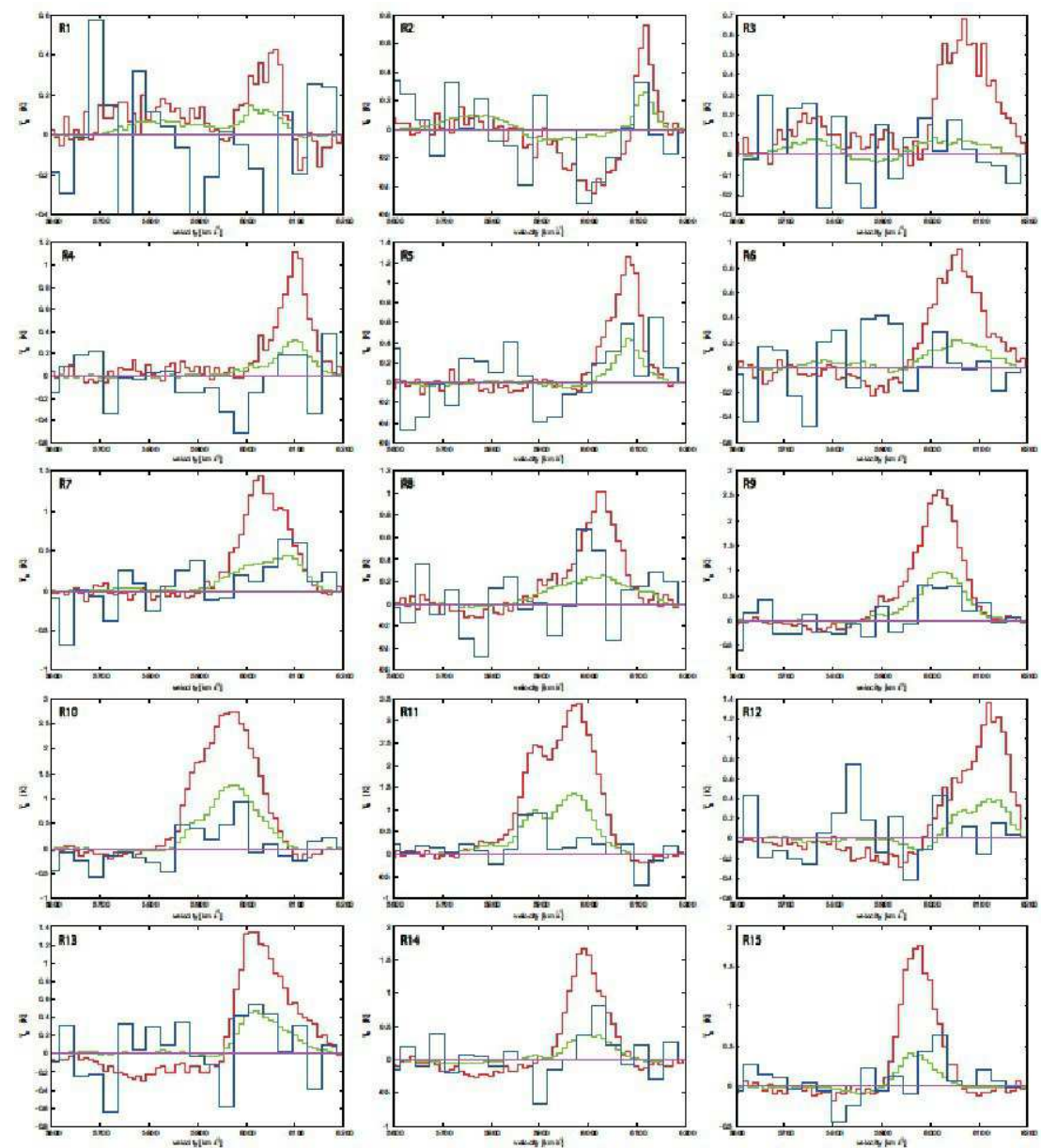

FIG. 19.- 2.'0 box-summed spectra of ${ }^{12} \mathrm{CO}(1-0)$ (red line), ${ }^{13} \mathrm{CO}(1-0) \times 10$ (green broken line), and ${ }^{12} \mathrm{CO}(3-2)$ (blue broken line) at the each box, labeled R1 - R39 of Fig 7 . The spectra are taken from the ALMA data cubes after correcting the cubes for the primary beam attenuation and convolving them to $2{ }^{\prime \prime} 0 \times 1{ }^{\prime \prime} 5$ resolution (P.A. $=83 \mathrm{deg}$ ). 

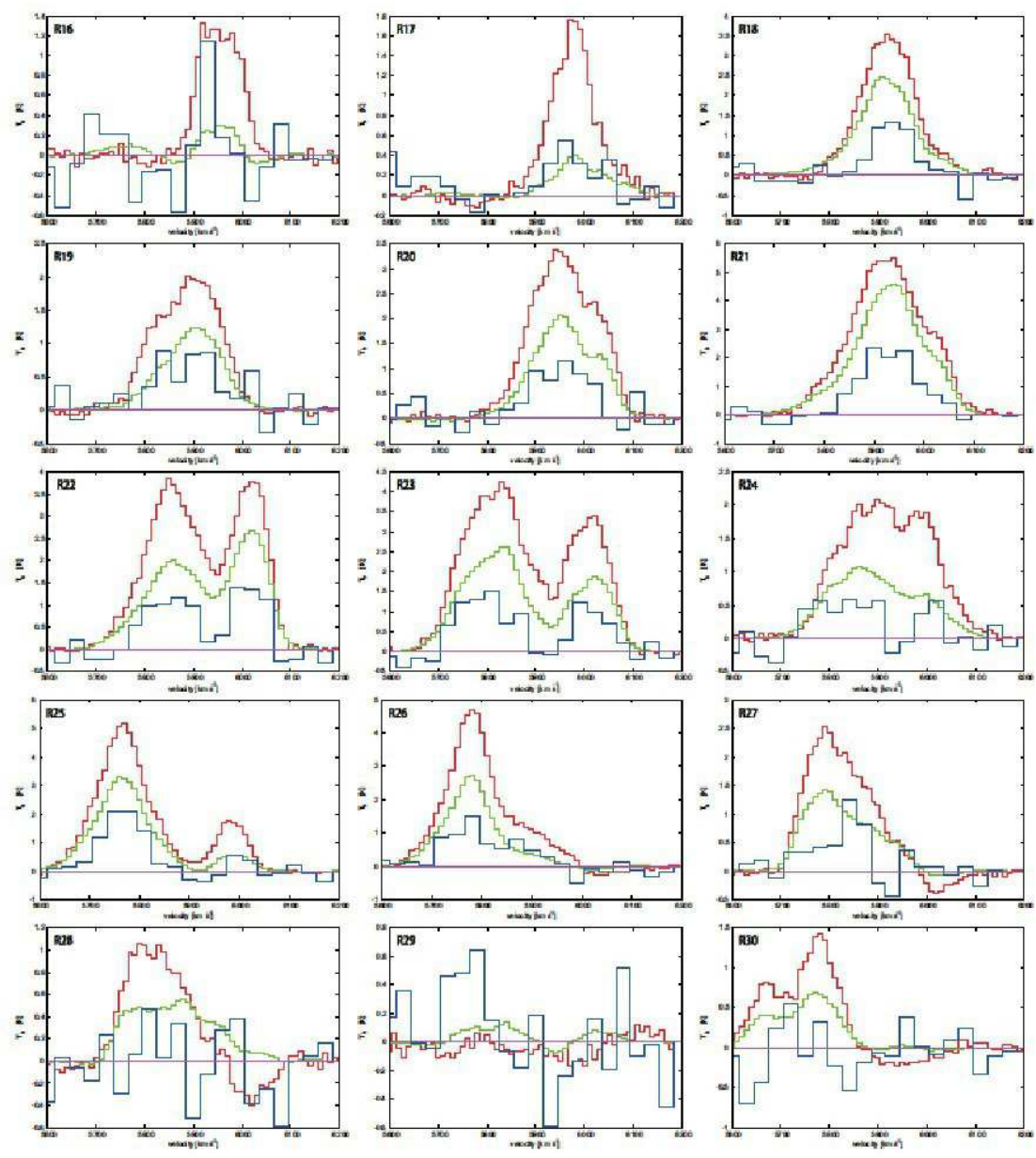

FIG. 19.- continued.
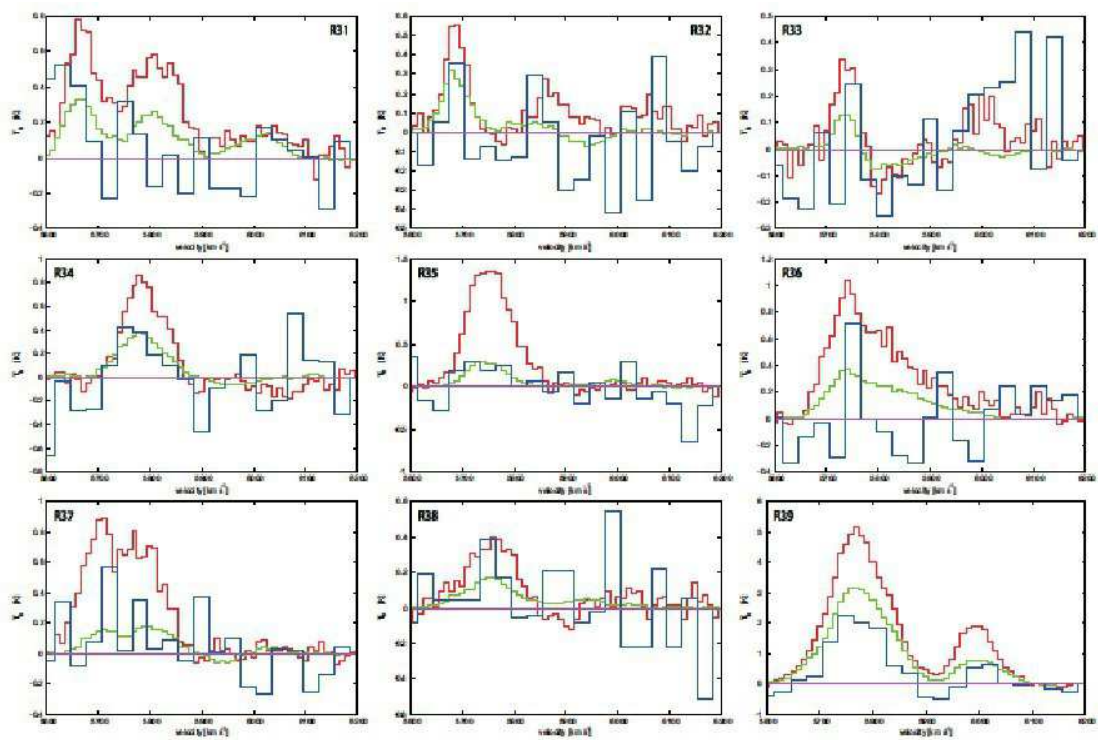

FIG. 19.- continued. 

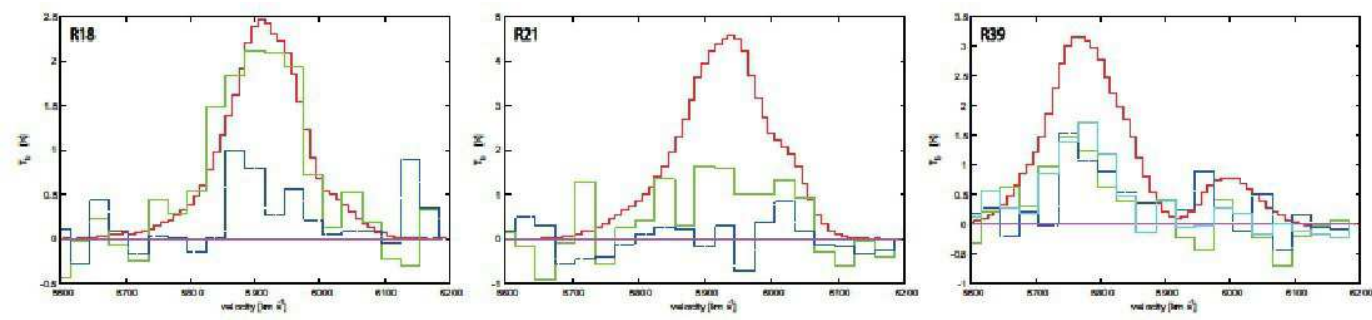

FIG. 20. - 2'. 0 box-summed spectra of ${ }^{12} \mathrm{CO}(3-2)$ (red line), CN $\left(1_{3 / 2}-0_{1 / 2}\right) \times 10$ (green broken line), CS $(2-1) \times 10$ (blue broken line), and $\mathrm{CH}_{3} \mathrm{OH}\left(2_{k}-1_{k}\right) \times 10$ (light blue broken line) at R18, R21, and R39, of Fig 7 . The spectra are taken from the ALMA data cubes after correcting the cubes for the primary beam attenuation and convolving them to $2{ }^{\prime \prime} 0 \times 11^{\prime \prime} 5$ resolution (P.A. $\left.=83 \mathrm{deg}\right)$.
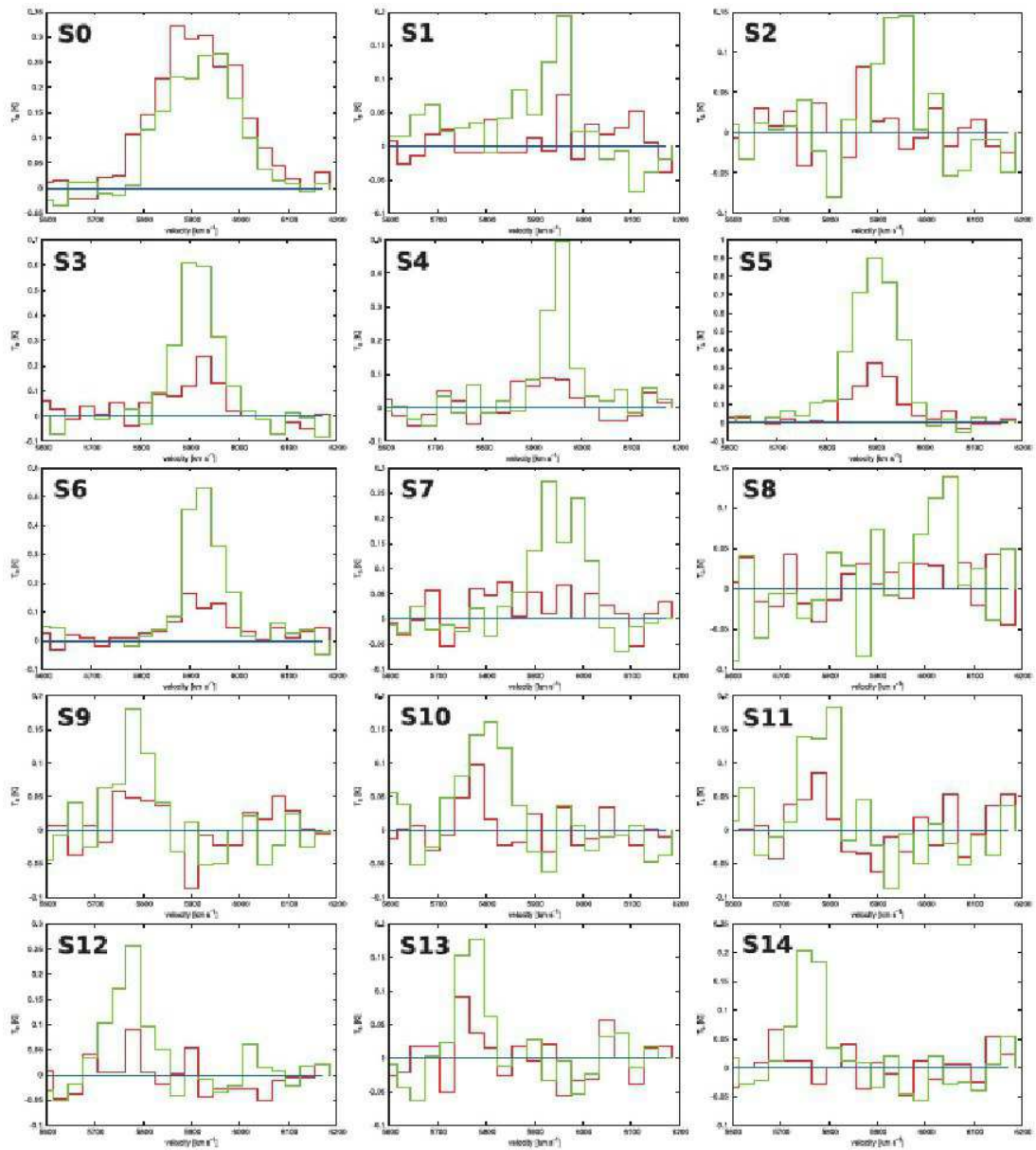

FIG. 21. - $0^{\prime \prime} 5$ box-summed spectra of $\mathrm{HCN}(4-3)$ (red line) and $\mathrm{HCO}^{+}(4-3)$ (green broken line) at the each box, labeled $\mathrm{S} 0-\mathrm{S} 14$, of Fig 7. 